Otimização de operações de entrada e saída visando reduzir o tempo de resposta de aplicações distribuídas que manipulam grandes volumes de dados 



\section{Otimização de operações de entrada e saída visando reduzir o tempo de resposta de aplicações distribuídas que manipulam grandes volumes de dados}

Renato Porfirio Ishii

Orientador: Prof. Dr. Rodrigo Fernandes de Mello

Tese apresentada ao Instituto de Ciências Matemáticas e de Computação - ICMC-USP, como parte dos requisitos para obtenção do título de Doutor em Ciências - Ciências de Computação e Matemática Computacional. 

À minha esposa Umarla e à nossa princesa Isabele, com amor, admiração e gratidão por sua compreensão, carinho, presença e incansável apoio ao longo do período de elaboração deste trabalho. 


\section{Agradecimentos}

A Deus por sua imensa bondade, generosidade e por todas as graças derramadas sobre a minha vida.

Aos meus pais Fumio e Iracema, à minha irmã Regina, por todo amor, dedicação e por serem fortaleza em tantos momentos difíceis. Por não medirem esforços e dedicação para eu realizar meus estudos, meu muito obrigado!

Agradeço em especial ao professor Rodrigo Mello, sempre alegre, entusiasmado, motivado e dedicado ao seu trabalho, um exemplo a ser seguido. Agradeço pela confiança, paciência infinita e orientação. Obrigado por tantos ensinamentos e pela amizade.

Às professoras Agma e Maria Cristina pelo apoio e credibilidade em nosso trabalho. Por terem conduzido sábia e prontamente, talvez, uma das etapas mais críticas de elaboração deste trabalho. Agradeço também à Cláudia Mello, pelo apoio incondicional e pela amizade.

Aos colegas de grupo: Ricardo Rios, Marcelo, Eugeni, Augusto, Paulo, Cássio, Eduardo, Vinicius e ao professor Leandro Franco pelas várias discussões e contribuições sempre tão valiosas. Ao Luciano, Márcio e aos colegas de grupo por se prontificarem a revisar o texto desta tese.

Aos amigos de todas as horas, sempre dispostos a ajudar, Rodrigo Pedra, Rodrigo Calvo, Bruno Feres, Adriano, Marcelo (K1), Nazira, Patrícia, Menotti, Juliano, Caio e Michel. Ao Roberto que sempre foi um irmão para mim durante toda minha estada em São Carlos. Ao Verardi que abriu as portas de sua casa para me receber tão bem. Ao casal Luciano e Lilian que foram fantásticos em, gentilmente, nos conceder sua casa. Ao Douglas e ao Vinícius que foram os principais responsáveis e incentivadores para que eu iniciasse meus estudos em São Carlos.

A todos os meus familiares, especialmente, aos meus sobrinhos Luiz Fernando e João Paulo, Seu Umbelino e Dona Marli, que sempre nos deram total apoio, incentivo e por compreenderem nossa ausência.

Aos amigos da FACOM que possibilitaram meu afastamento durante a fase final de elaboração deste trabalho.

À UFMS e à FUNDECT pelo apoio financeiro. À CAPES e ao CNPq pelo custeio de viagens e participação em conferência. 


\section{Resumo}

Aplicações científicas atuais têm produzido volumes de dados cada vez maiores. O processamento, a manipulação e a análise desses dados requerem infraestruturas computacionais de larga escala tais como aglomerados e grades de computadores. Nesse contexto, várias pesquisas visam o aumento de desempenho dessas aplicações por meio da otimização de acesso a dados. Para alcançar tal objetivo, pesquisadores têm utilizado técnicas de replicação, migração, distribuição e paralelismo de dados. No entanto, uma das principais lacunas dessas pesquisas está na falta de emprego de conhecimento sobre aplicações com objetivo de realizar essa otimização. Essa lacuna motivou esta tese que visa empregar comportamento histórico e preditivo de aplicações a fim de otimizar suas operações de leitura e escrita sobre dados distribuídos. Os estudos foram iniciados empregando-se informações previamente monitoradas de aplicações a fim de tomar decisões relativas à replicação, migração e manutenção de consistência. Observou-se, por meio de uma nova heurística, que um conjunto histórico de eventos auxilia a estimar o comportamento futuro de uma aplicação e otimizar seus acessos. Essa primeira abordagem requer ao menos uma execução prévia da aplicação para composição de histórico. Esse requisito pode limitar aplicações reais que apresentam mudanças comportamentais ou que necessitam de longos períodos de execução para completar seu processamento. Para superar essa limitação, uma segunda abordagem foi proposta baseada na predição on-line de eventos comportamentais de aplicações. Essa abordagem não requer a execução prévia da aplicação e permite adaptar estimativas de comportamento futuro em função de alterações adjacentes. A abordagem preditiva analisa propriedades de séries temporais com objetivo de classificar seus processos geradores. Essa classificação aponta modelos que melhor se ajustam ao comportamento das aplicações e que, portanto, permitem predições com maior acurácia. As duas abordagens propostas foram implementadas e avaliadas utilizando o simulador OptorSim, vinculado ao projeto LHC/CERN, amplamente adotado pela comunidade científica. Experimentos constataram que as duas abordagens propostas reduzem o tempo de resposta (ou execução) de aplicações que manipulam grandes volumes de dados distribuídos em aproximadamente $50 \%$.

Palavras-chave: Computação distribuída. Sistema de arquivos distribuídos. Otimização de acesso a dados. Análise de séries temporais. 


\section{Abstract}

Current scientific applications produce large amount of data and handling, processing and analyzing such data require large-scale computing infrastructure such as clusters and grids. In this context, various studies have focused at improving the performance of these applications by optimizing data access. In order to achieve this goal, researchers have employed techniques of replication, migration, distribution and parallelism of data. However, these common approaches do not use knowledge about the applications at hand to perform this optimization. This gap motivated the present thesis, which aims at applying historical and predictive behavior of applications to optimize their reading and writing operations on distributed data. Based on information previously monitored from applications to make decisions regarding replication, migration and consistency of data, a new heuristic was initially proposed. Its evaluation revealed that considering sets of historical events indeed helps to estimate the behavior of future applications and to optimize their access operations. Thus it was embedded into two optimization approaches. The first one requires at least a previous execution for the history composition. This requirement may limit real world applications which present behavioral changes or take very long time to execute. In order to overcome this issue, a second technique was proposed. It performs on-line predictions about the behavior of the applications, mitigating the need of any prior execution. Additionally, this approach considers the future behavior of an application as a function of its underlying changes. This behavior can be modeled as time series. The method works by analyzing the series properties in order to classify their generating processes. This classification indicates models that best fit the applications behavior, allowing more accurate predictions. Experiments using the OptorSim simulator (LHC/CERN project) confirmed that the proposed approaches are able to reduce the response time of applications that handle large amount of distributed data in approximately $50 \%$.

Keywords: Distributed computing. Distributed file system. Data access optimization. Time series analysis. 


\section{Lista de Figuras}

3.1 Representação de uma série temporal. Para cada instante de tempo $t$, tem-se uma variável aleatória $X(t, \omega)$, com uma f.d.p. $f_{x}(X)$ (Morettin e Toloi, 2006). . . . . . . . . . . . . 17

3.2 Uma taxonomia que relaciona os principais critérios considerados na análise de séries temporais, bem como as principais ferramentas que podem ser utilizadas na modelagem e avaliação. . . . . . . . . . . . . . . . . 28

3.3 Duas representações para o Sistema Rössler: a primeira ilustra como as observações estão dispostas no tempo (a) e a segunda (b), após sua reconstrução em espaço multidimensional. . . . . . 31

3.4 Uma diagonal no RP corresponde a uma seção da trajetória (linha tracejada) que permanece próxima à outra seção (linha sólida) ao redor de uma $\varepsilon$-vizinhança (Marwan et al., 2007). . . . . . . . . . . 33

3.5 Uma representação gráfica de Recurrence Plot do sistema Rössler obtida a partir de sua matriz de recorrências. . . . . . . . . . . 34

3.6 Um exemplo de histograma das diagonais de um RP. . . . . . . . 36

3.7 Gráfico RP da série Caminhada Aleatória. . . . . . . . . . . . . . 39

3.8 Gráfico RP da série AR(1). . . . . . . . . . . . . . . . . . . . . . 39

3.9 Gráfico RP da série ARIMA $(1,1,1) \ldots \ldots \ldots$. . . . . . . . . . . 40

3.10 Gráfico RP da série mapa Logístico. . . . . . . . . . . . . . . 41

3.11 Gráfico RP da série atrator de Lorenz. . . . . . . . . . . . . . . 42

$3.12 \mathrm{Um}$ exemplo de rede neural supervisionada com uma única camada escondida. . . . . . . . . . . . . . 43

3.13Teste de estacionariedade para série temporal Caminhada

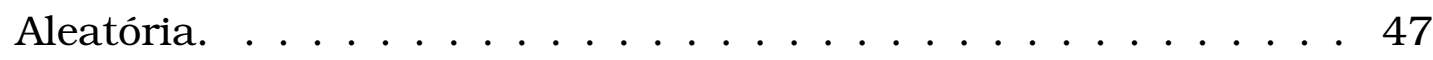

3.14 Teste de estacionariedade para série temporal AR(1). . . . . . . . 48

3.15Teste de estacionariedade para série temporal ARIMA $(1,1,1)$. . . 49

3.16 Teste de estacionariedade para o mapa Logístico. . . . . . . . . . 49

3.17Teste de estacionariedade para o atrator de Lorenz. . . . . . . . 50 
3.18 Gráfico RP da série proj. . . . . . . . . . . . . . . . . . 52

3.19 Gráfico RP da série hm. . . . . . . . . . . . . . . . . . 52

3.20 Gráfico RP da série mds. . . . . . . . . . . . . . . . . . . 53

3.21 Teste de estacionariedade para a série proj. . . . . . . . . . 54

3.22 Teste de estacionariedade para a série hm. . . . . . . . . . 54

3.23Teste de estacionariedade para a série mds. . . . . . . . . . 55

3.24 Série temporal proj: resultados da métrica NRMSE. . . . . . . 56

3.25 Série temporal $\mathbf{h m}$ : resultados da métrica NRMSE. . . . . . . . . 59

3.26 Série temporal mds: resultados da métrica NRMSE. . . . . . . . . 61

4.1 Exemplo de um ambiente distribuído com respectivos elementos de processamento e rede de comunicação. . . . . . . . . . . . 67

4.2 Descrição da metodologia proposta para otimização de acesso a dados composta por três módulos: base de conhecimento, janela deslizante e heurística. . . . . . . . . . . . . . . . . . 75

4.3 Gráfico com resultados de experimentos comparativos entre estratégias LRU, LFU, ECO e HEU com janela de comprimento fixo. É interessante observar os impactos proporcionados com a variação no comprimento da janela. . . . . . . . . . . . . . 77

4.4 Exemplo de funcionamento da abordagem JDA $\operatorname{com} \beta=0,10 \ldots \quad$. 79

4.5 Exemplo de transferência completa de um arquivo. . . . . . . . 80

4.6 Exemplo de transferência parcial de um arquivo. . . . . . . . . . 80

4.7 Arquitetura do simulador OptorSim para grades computacionais que suporta modelos de replicação de dados. . . . . . . . . . . . 84

4.8 Resultados do experimento que considera um ambiente com $100 \%$ de operações de leitura. . . . . . . . . . . . . . . . . . 89

4.9 Resultados do experimento que considera um ambiente com $5 \%$ de operações de escrita e $95 \%$ de operações de leitura. . . . . . . . 90

4.10Resultados do experimento que considera um ambiente com $95 \%$ de operações de escrita e $5 \%$ de operações de leitura. . . . . . . 91

4.11 Etapas da abordagem baseada em predição on-line. . . . . . . . 92

4.12 Exemplo de execução baseada em predição on-line. . . . . . . . . 95

4.13Resultados comparativos entre as técnicas de otimização LRU,

LFU, ECO, H-Hist e H-Pred para o traço de execução hm. . . . . . 95

4.14 Resultados comparativos entre as técnicas de otimização LRU, LFU, ECO, H-Hist e H-Pred para o traço de execução mds. .. . 96

4.15Resultados comparativos entre as técnicas de otimização LRU,

LFU, ECO, H-Hist e H-Pred para o traço de execução proj. . . . . 97

4.16Resultados comparativos entre as técnicas de otimização LRU, LFU, ECO, H-Hist e H-Pred para o traço de execução proj com modificações. . . . . . . . . . . . . . . . . . . 97 
A.1 Operador crossover . . . . . . . . . . . . . . . . . . 120

A.2 Problema de busca ao alimento. . . . . . . . . . . . . . 121 


\section{Lista de Tabelas}

3.1 Conjunto de cinco séries temporais para estudo. . . . . . . . . 30

3.2 Valores de RR para cinco séries temporais. . . . . . . . . . . 35

3.3 Valores de DET para cinco séries temporais com $L_{\min }=2$. . . . . 37

3.4 Valores de DIV para as cinco séries temporais em estudo. . . . . . 37

3.5 Valores de ENTR para as cinco séries temporais em estudo. . . . . 38

3.6 Valores das medidas RQA para série Caminhada Aleatória com dimensão de separação 1 , embutida 3 e distância $\varepsilon=1,0 . \ldots .39$

3.7 Valores das medidas RQA para série AR(1) com dimensão de separação 3 , embutida 5 e distância $\varepsilon=1,0 \ldots \ldots$. . . . . . 39

3.8 Valores das medidas RQA para série $\operatorname{ARIMA}(1,1,1)$ com dimensão de separação 12 , embutida 5 e distância $\varepsilon=1,0 \ldots \ldots \ldots$. . . 40

3.9 Valores das medidas RQA para série mapa Logístico com dimensão de separação 3 , embutida 5 e distância $\varepsilon=1,0 . \ldots$. . 41

3.10Valores das medidas RQA para série atrator de Lorenz com dimensão de separação 5 , embutida 3 e distância $\varepsilon=1,0 . \ldots$. . 42

3.11 Resultados do teste WNN para identificação linearidade em cinco séries temporais. . . . . . . . . . . . . . . . 45

3.12 Característica do sistema utilizado no experimentos. Um Data Center com: 13 servidores, 36 volumes lógicos e 179 discos rígidos. 51

3.13 Medidas RQA para a série proj com dimensão de separação 6, embutida 14 e $\varepsilon=1,0 \ldots \ldots \ldots \ldots$. . . . . . . . . . 52

3.14 Medidas RQA para a série $\mathbf{h m}$ com dimensão de separação 4, embutida 2 e $\varepsilon=1,0 \ldots \ldots \ldots \ldots \ldots$. . . . . . . . . 52

3.15Medidas RQA para a série mds com dimensão de separação 5, embutida 2 e $\varepsilon=1,0 \ldots \ldots \ldots \ldots \ldots \ldots$

3.16Resultados da aplicação do teste de linearidade WNN sobre três séries temporais obtidas a partir de traços de execução do repositório SNIA. . . . . . . . . . . . . . . . . . . 53

$3.17 \mu$ e $\sigma$ de NRMSE para técnica ARMA(1,2) e AR(1) . . . . . . . . 57 
$3.18 \mu$ e $\sigma$ de NRMSE para ARMA(1,2) e Polynom. . . . . . . . . 58

$3.19 \mu$ e $\sigma$ de NRMSE para ARMA(1,2) e RBF. . . . . . . . . . 58

$3.20 \mu$ e $\sigma$ de NRMSE para ARIMA(2,1,2) e AR(1). . . . . . . . . . 59

$3.21 \mu$ e $\sigma$ da NRMSE para ARIMA(2,1,2) e Polynom. . . . . . . . . 60

$3.22 \mu$ e $\sigma$ da NRMSE para as técnicas Espaço Fase/RBF e AR(1). . . 61

$3.23 \mu$ e $\sigma$ da NRMSE para as técnicas Espaço Fase/RBF e Polynom. . 62

$3.24 \mu$ e $\sigma$ da NRMSE para as técnicas Espaço Fase/RBF e ARMA(5,5). 62

4.1 Comportamento de Processos . . . . . . . . . . . 66

4.2 Características dos elementos de processamento da grade apresentada na Figura 4.1. . . . . . . . . . . . . . 67

4.3 Espaço de soluções para o problema de distribuição de arquivos sobre os elementos de processamento de uma grade. . . . . . . 68

4.4 Parâmetros utilizados na definição do problema de acesso a dados. 71

4.5 Exemplo de um arquivo de traços de execução, onde cada linha representa uma operação do processo $p_{i}$ sobre um arquivo identificado por $f_{j} \ldots \ldots \ldots \ldots \ldots \ldots \ldots \ldots \ldots$

4.6 Resultados para captura de informações de processos através de dois mecanismos: Ptrace e DLSym, considerando dois benchmarks: Nbench e Bonnie . . . . . . . . . . . . 88

4.7 Exemplo de informações utilizadas na otimização de acesso por meio da abordagem baseada em predição on-line. . . . . . . . . 94 


\section{Lista de Algoritmos}

1 Procedimento Recupera $(f) \ldots \ldots \ldots$. . . . . . . . 81

2 Procedimento Leitura(tr) . . . . . . . . . . . . . . 81

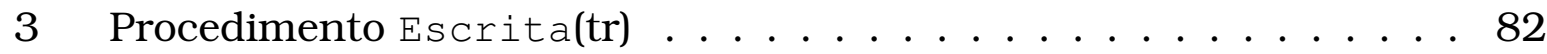

4 Procedimento Invalida $(f) \ldots \ldots \ldots \ldots$. . . . . . . 82

5 Heurística baseada em comportamento histórico . . . . . . . . . 83

6 Heurística baseada em comportamento preditivo . . . . . . . . . . 93 


\section{Lista de Abreviaturas}

ACF Auto Correlation Function

AMI Auto Mutual Information

AR Auto Regressive Processes

ARCH Auto Regressive Conditional Heteroskedasticity

ARIMA Auto Regressive Integrated Moving Average Processes

ARMA Auto Regressive and Moving Average Processes

BIOCOM The BIOinspired COMputation Laboratory

CERN Conseil Européen pour la Recherche Nucléaire ou European Organization for Nuclear Research

DET DETerminism rate

DIV DIVergence rate

EDG European Data Grid

ENTR Shannon ENTRopy

EP Elemento de Processamento

FNN False Nearest Neighbors

ICMC Instituto de Ciências Matemáticas e de Computação

JDA Janela Deslizante Adaptativa

KNN $\quad \mathbf{K}$ - Nearest Neighbor

LCG Large Hadron Collider Computing Grid

LFU Least Frequently Used

LHC Large Hadron Collider

LOI Line Of Identity

LRU Least Recently Used

MA Moving Average Processes

MSDT Multi-Source Data Transfer

PAD Problema de Acesso a Dados

PCA Principal Component Analysis

RBF Radial Basis Function

RP Recurrence Plot 
RQA Recurrence Quantification Analysis

RR Recurrence Rate

SMART Self-Monitoring Analysis and Reporting Technology

SNIA Storage Networking Industry Association

SONDE Self-Organizing Neural Network for DEtecting Novelties

STP Space-Time-Separation Plot

WNN White Neural Network 


\section{Sumário}

1 Introdução 1

1.1 Contextualização . . . . . . . . . . . . . . . 1

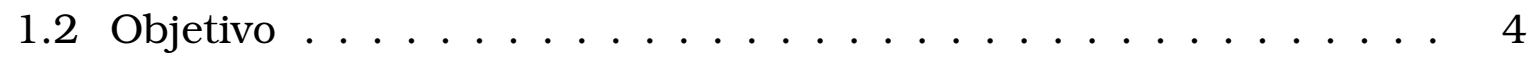

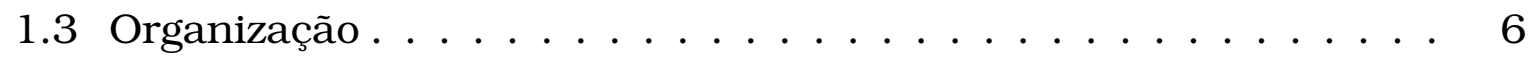

2 Trabalhos Relacionados $\quad 7$

2.1 Considerações Iniciais . . . . . . . . . . . . . . . . 7

2.2 Abordagens para otimização de acesso a dados distribuídos . . . 7

2.3 Predição do comportamento de processos . . . . . . . . . . 10

2.4 Análise dos trabalhos relacionados . . . . . . . . . . . 11

2.4.1 Sobre as abordagens para otimização de acesso a dados . . 11

2.4.2 Sobre a predição do comportamento de processos . . . . . 12

2.5 Considerações Finais . . . . . . . . . . . . . . . . . 13

3 Uma Metodologia para Classificação de Séries Temporais 15

3.1 Considerações Iniciais . . . . . . . . . . . . . . . 15

3.2 Revisão de séries temporais . . . . . . . . . . . . . 17

3.3 Técnicas de Modelagem . . . . . . . . . . . . . 21

3.3.1 Modelagem baseada em ferramentas determinísticas . . . . 21

3.3.2 Modelagem baseada em ferramentas estocásticas . . . . . . 25

3.4 Classificação dos processos geradores de séries temporais . . . 27

3.5 Avaliação dos processos geradores de séries temporais . . . . . 29

3.5.1 Avaliação de estocasticidade . . . . . . . . . . . . . 29

3.5.2 Avaliação de linearidade . . . . . . . . . . . . . . . . 42

3.5.3 Avaliação de estacionariedade . . . . . . . . . . . . . . 45

3.6 Experimentos com dados reais . . . . . . . . . . . . . . 50

3.7 Avaliação da modelagem . . . . . . . . . . . . . 55

3.8 Considerações Finais .................... 63 
4 Abordagens para o Problema de Acesso a Dados 65

4.1 Considerações Iniciais . . . . . . . . . . . . . . . . 65

4.2 Problema de acesso a dados: Definição Empírica . . . . . . . . 66

4.3 Problema de acesso a dados: Definição Formal . . . . . . . . . 68

4.4 Primeira abordagem proposta: utilização de informações históricas 74

4.4.1 Captura e composição da base de conhecimento . . . . . 75

4.4 .2 Janela deslizante adaptativa . . . . . . . . . . . 76

4.4 .3 Heurística proposta . . . . . . . . . . . . 79

4.4 .4 Experimentos . . . . . . . . . . . . . . 82

4.4 .5 Extensões do OptorSim . . . . . . . . . . . . 86

4.4.6 Parametrização dos experimentos . . . . . . . . . . 87

4.4.7 Avaliação de intrusão na captura de informações . . . . . 88

4.4.8 Resultados da primeira abordagem . . . . . . . . . 89

4.5 Segunda abordagem proposta: predição on-line de comportamento de processos . . . . . . . . . . . . . 92

4.5.1 Resultados da segunda abordagem . . . . . . . . . . 94

4.6 Considerações Finais . . . . . . . . . . . . . . . 98

5 Conclusão 101

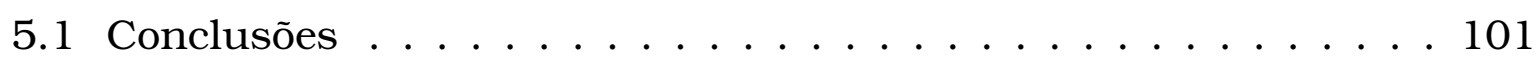

5.2 Contribuições . . . . . . . . . . . . . . . . . . 102

5.2.1 Relação com demais trabalhos do grupo de pesquisa . . . . 103

5.3 Dificuldades e principais problemas . . . . . . . . . . . . . 104

5.4 Propostas para trabalhos futuros . . . . . . . . . . 105

$\begin{array}{ll}\text { Referências Bibliográficas } & 107\end{array}$

A Metaheuristicas $\quad 119$

A.1 Algoritmos genéticos . . . . . . . . . . . . . . . . 119

A.2 Colônia de formigas . . . . . . . . . . . . . . . . . . 120

A.3 Simulated Annealing . . . . . . . . . . . . . . . . . . . . 122 


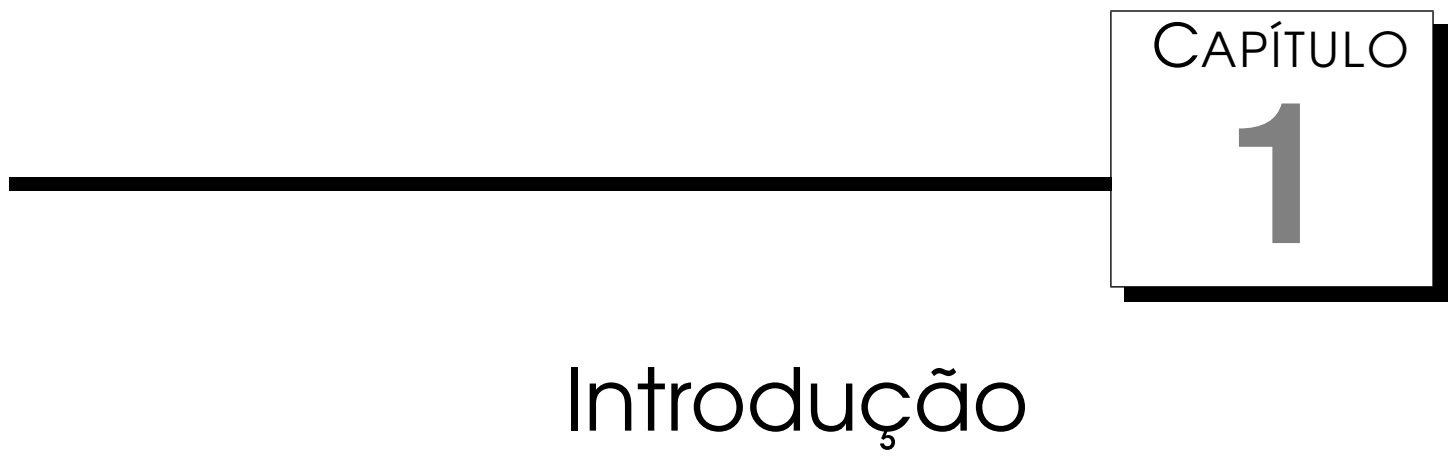

\subsection{Contextualização}

Até o Século XX a ciência foi predominantemente constituída por três paradigmas fundamentais: o empírico, o teórico e o computacional (Nielsen, 2009; Collins, 2010). Há milhares de anos, a ciência caracterizava-se basicamente por observações e análises de fenômenos da natureza, o que define o paradigma empírico. Centenas de anos atrás começaram a surgir os primeiros teoremas e generalizações, tais como as Leis de Kepler, Newton e as Equações de Maxwell, caracterizando o paradigma teórico. Entretanto, há algumas décadas e para muitos problemas desta atual geração, os modelos teóricos tornaram-se muito difíceis de serem resolvidos analiticamente, devido ao volume de parâmetros envolvidos. Esse aspecto motivou o desenvolvimento de simuladores, marco histórico do paradigma computacional.

Modelos resolvidos por simulação podem manipular e gerar grandes volumes de dados. Diversas infraestruturas que suportam a realização desses tipos de experimentos têm sido desenvolvidas. Exemplos disso são os projetos Pan-STARRS $^{1}$, que captura 2,5 petabytes (PB) de dados por ano e o Large Hadron Collider (LHC) ${ }^{2}$, que gera entre 50 a $100 \mathrm{~PB}$ de dados por ano (Gray, 2009).

Essa tendência para o paradigma computacional confirma que as pesquisas científicas têm sido desenvolvidas de maneira diferente, pois a computação permitiu considerar e estudar um maior número de variáveis e volume de dados (Nielsen, 2009; Collins, 2010). Nessa nova abordagem

\footnotetext{
${ }^{1}$ http://pan-starrs.ifa.hawaii.edu/public

${ }^{2}$ http://public.web.cern.ch/public/en/LHC/LHC-en.html
} 
científica, dados são capturados por instrumentos (telescópios, aceleradores de partículas, eletroencefalogramas, etc.) ou gerados por simulações. Em seguida, esses dados são processados por software específicos, a fim de extrair informações relevantes e resumir cenários de interesse. Somente após esses passos, cientistas analisam resultados e obtêm conclusões. As técnicas, modelos e métodos utilizados para esse nicho da ciência requerem a manipulação de grandes volumes de dados, o que, segundo alguns pesquisadores, caracteriza um quarto paradigma científico (Collins, 2010).

Nesse contexto, a computação distribuída é empregada com objetivo de utilizar vários recursos computacionais de larga escala e atender aplicações de altas demandas por processamento e acesso a dados. A emergência da Internet e de suas tecnologias subjacentes, bem como o desenvolvimento da microeletrônica e das redes de alto desempenho, permite a construção de infraestruturas computacionais de larga escala e com grande potencial para solucionar problemas dessa nova abordagem científica (Parashar e Hariri, 2007).

Os principais representantes dessas infraestruturas computacionais de larga escala são os aglomerados (clusters) e, mais recentemente, as grades computacionais (grids). Aglomerados são compostos por um conjunto de estações de trabalho ou computadores pessoais de arquitetura semelhante, conectados por meio de uma rede local de alta velocidade, onde cada estação, geralmente, executa o mesmo sistema operacional. Grades são organizadas na forma de federações de computadores. Cada federação representa um domínio administrativo distinto. Esse tipo de organização caracteriza-se pela alta heterogeneidade de hardware e de software. Nessas infraestruturas, recursos de diferentes organizações são agregados, permitindo colaboração entre pessoas (pesquisadores) ou instituições (Stockinger, 2007).

Essas infraestruturas têm grande potencial para atender as demandas de aplicações que manipulam grandes volumes de dados, também denominadas data-intensive. No entanto, poucas abordagens foram desenvolvidas a fim de permitir e, efetivamente, auxiliar o processamento e acesso a grandes volumes de dados distribuídos. Com tal suporte, poder-se-ia atender os requisitos da ciência contemporânea e, portanto, auxiliar em avanços nas mais diversas áreas do conhecimento tais como dobramento de proteína (protein folding) na biologia molecular, física de partículas (high energy physics), modelagem do mercado financeiro (financial modeling), simulação de terremotos, modelagem climática e previsão do tempo, diagnóstico de propulsores de aeronaves, bioinformática, descoberta de novos medicamentos (drug discovery) e astrofísica (Hey e Trefethen, 2005; Ranjan et al., 2006; Gray, 2009; Collins, 2010). 
O projeto e desenvolvimento dessas abordagens introduziu novos desafios relacionados ao aumento de desempenho de aplicações que executam sobre infraestruturas distribuídas. Dentre esses desafios estão a dificuldade em gerenciar a utilização de recursos heterogêneos, em planejar e adaptar a execução de aplicações em função de latências de acesso variáveis, em detectar e recuperar sistemas sob falhas. Esses desafios motivaram pesquisas em áreas como: escalonamento de processos, balanceamento de carga, protocolos de comunicação e baixa latência, bibliotecas de comunicação, arquiteturas de hardware de alto desempenho e otimização de acesso a dados (Fox e Gannon, 2001; Ranjan et al., 2006). Dentre esses tópicos, a otimização de acesso a dados tem despertado grande interesse da comunidade científica, principalmente, por estar intimamente relacionado ao novo paradigma de manipulação de grandes volumes de dados (Gray, 2009; Nielsen, 2009; Collins, 2010).

A alta complexidade do problema de acesso a dados (PAD) está relacionada, principalmente, à localização e à manutenção de consistência desses dados. Além disso, outros fatores devem ser avaliados tais como a possibilidade de distribuição, migração, replicação de dados e impacto de operações de leitura e escrita no escalonamento de processos (Dang e Lim, 2007).

A distribuição de dados busca alocar, eficientemente, trechos (chunks) de arquivos sobre a infraestrutura computacional distribuída a fim de aumentar o desempenho das operações de leitura e escrita.

A migração deve analisar os impactos envolvidos na transferência de dados a fim de reduzir custos de operações de entrada e saída. Em geral essa abordagem é utilizada para aproximar dados e aplicações, visando a redução de custos de sincronismo. A migração deve considerar aspectos relativos à capacidade de armazenamento e localização de dispositivos, protocolos de comunicação, latência, largura de banda e topologia da infraestrutura.

A replicação decide sobre onde e quando copiar arquivos (criar réplicas) com objetivo de aumentar o desempenho do sistema e a resiliência a falhas (Rahman et al., 2005, 2007). Essa técnica deve considerar a latência, largura de banda e padrão de acesso a dados a fim de otimizar suas operações.

A consistência visa coordenar a atualização de múltiplas réplicas do mesmo arquivo (Sun e Xu, 2004), a fim de prover uma visão única dos dados para aplicações. Além desses aspectos, há ainda os impactos que a otimização de acesso a dados gera sobre o escalonamento de processos. Nesse contexto, políticas de escalonamento devem, também, considerar a localização dos dados, com o objetivo de reduzir o tempo de resposta (execução) de aplicações (Elghirani et al., 2007).

Esses fatores empregados com o objetivo de solucionar o problema de 
acesso a dados têm sido abordados em diferentes trabalhos (Oliker et al., 2004; Sun e Xu, 2004; Rahman et al., 2005; Oldfield e Kotz, 2006; Dang e Lim, 2007; Elghirani et al., 2007; Sato et al., 2009; Kim et al., 2009; Chervenak et al., 2009; AL-Mistarihi e Yong, 2009), discutidos no Capítulo 2. No entanto, segundo estudos conduzidos no contexto desta tese, eles apresentam três principais limitações. A primeira limitação indica que grande parte dos trabalhos consideram exclusivamente operações de leitura, o que tende a restringir seu uso em aplicações reais (Bell et al., 2003). O foco em operações de leitura se deve à complexidade da manutenção de consistência dos dados. Como segunda limitação, esses trabalhos empregam abordagens estáticas para otimizar acessos, ou seja, mecanismos que não se adaptam à dinâmica comportamental do ambiente (entrada e saída de usuários, inserção e remoção de computadores no sistema e variação comportamental de processos de aplicações ${ }^{3}$ ). A última limitação advém do fato que esses trabalhos pouco abordam o desempenho global do sistema, o qual depende do padrão de acesso de cada processo individual aos dados.

Nesse contexto, é de suma importância entender, analisar, estimar e/ou predizer o comportamento de processos como forma de otimizar suas operações de leitura e escrita em dados, visando reduzir seus tempos de resposta (execução) e, consequentemente, aumentar o desempenho global do sistema.

Essas lacunas existentes motivaram esta tese cuja hipótese é: a antecipação de operações de leitura e escrita, por meio de estimativas, predições e análise de séries temporais, pode aumentar o desempenho de aplicações que manipulam grandes volumes de dados.

Essa hipótese está pautada em pesquisas anteriores desenvolvidas pelo grupo de pesquisa, vinculado a esta tese, as quais comprovaram aumento significativo de desempenho de aplicações. Nessas pesquisas, o comportamento de aplicações auxilia na otimização do escalonamento de processos (Dodonov, 2009; de Mello, 2009; Dodonov e de Mello, 2010).

\subsection{Objetivo}

As limitações de trabalhos relacionados e os bons resultados previamente obtidos pelo grupo de pesquisa, na área de escalonamento de processos, motivaram esta tese que visa empregar comportamento histórico e preditivo de aplicações a fim de otimizar suas operações de leitura e escrita sobre dados distribuídos. Como resultado final da otimização, espera-se reduzir o tempo de resposta (execução) de aplicações que manipulam grandes volumes de dados.

\footnotetext{
${ }^{3}$ Aplicações são constituídas por um ou mais processos. Além disso, cada processo realiza um certo número de operações de leitura e escrita sobre dados distribuídos.
} 
Para alcançar esse objetivo, estudos foram iniciados com o monitoramento de informações de aplicações (consumo de recursos) a fim de compor uma base de conhecimento histórica. Essa base foi, posteriormente, utilizada para estudar o comportamento de aplicações e projetar abordagens de otimização de acesso a dados distribuídos.

$\mathrm{Na}$ próxima etapa, foram estudadas e exploradas meta-heurísticas tais como Algoritmos Genéticos (Goldberg, 1989), Colônia de Formigas (Dorigo e Di Caro, 1999), Simulated Annealing (Kirkpatrick et al., 1983) e Redes Neurais de Hopfield (Hopfield, 1982), com o objetivo de propor uma abordagem de otimização. No entanto, foi constatado que essas técnicas não apresentaram resultados convincentes. Ao analisar esses resultados, observou-se que a otimização tendia para comportamento médio de acesso e não tratava eventos específicos de leitura e escrita de dados no tempo.

Para superar essa limitação foi proposta uma primeira heurística que busca otimizar o acesso a dados por meio da estimação de eventos futuros de leitura e escrita. Essa estimação é realizada considerando comportamentos previamente monitorados de determinada aplicação. Nesse cenário, analisa-se o momento atual de execução de uma aplicação, o qual é comparado ao histórico e, a partir disso, obtém-se uma estimativa dos próximos eventos daquela aplicação. Caso a aplicação seja estável, isto é, não apresente variações comportamentais significativas, esta técnica oferece bons resultados.

Essa primeira abordagem apresenta três limitações. A primeira se refere à necessidade de uma execução prévia da aplicação para a composição de histórico. A segunda ocorre devido a variações comportamentais, as quais prejudicam boas estimativas. A terceira exige que informações históricas sejam armazenadas e, sistematicamente processadas, o que pode ser oneroso (em termos de espaço e tempo) para o desempenho da abordagem. Essas limitações foram, posteriormente, consideradas em uma segunda abordagem de otimização que utiliza técnicas de análise de séries temporais para realizar a predição on-line de eventos de leitura e escrita.

Essa última abordagem não necessita de uma execução prévia da aplicação, mas apenas de algumas observações anteriores sobre seu comportamento. Em seguida, essas observações são organizadas na forma de séries temporais e analisadas com objetivo de classificar seus processos geradores (Mello e Yang, 2009). Essa classificação aponta modelos mais adequados para predizer eventos futuros de leitura e escrita. Esses eventos são, posteriormente, considerados pela mesma heurística desenvolvida na etapa anterior, a fim de otimizar o acesso a dados distribuídos.

Para validar as duas abordagens de otimização propostas (baseada em 
histórico e preditiva), foram conduzidos experimentos utilizando o simulador OptorSim (Bell et al., 2003), vinculado ao projeto LHC - European Organization for Nuclear Research (CERN), amplamente adotado na literatura (Kim et al., 2009; Bell et al., 2003). Experimentos constataram que ambas abordagens propostas reduzem o tempo de reposta médio de aplicações que manipulam grandes volumes de dados em aproximadamente $50 \%$.

\subsection{Organização}

Esta tese de doutorado está organizada da seguinte maneira: O Capítulo 2 apresenta e discute trabalhos relacionados ao problema de acesso a dados, bem como pesquisas que utilizam predição de comportamento de processos. O Capítulo 3 apresenta estudos sobre análise de séries temporais e testes de aderência para a avaliação de propriedades relativas à estocasticidade, linearidade e estacionariedade. Em seguida, é proposta uma metodologia para a classificação de séries temporais, indicando modelos que melhor se ajustam ao comportamento dos dados. Para fins de validação, os modelos obtidos são aplicados com objetivo de predizer eventos de leitura e escrita. Experimentos sobre conjuntos de dados reais, obtidos a partir do repositório Storage Networking Industry Association (SNIA) (Narayanan et al., 2008), confirmam os bons resultados provenientes da metodologia de classificação de séries temporais proposta nesse capítulo. No Capítulo 4 são apresentadas as duas abordagens propostas para o problema de acesso a dados: baseada no comportamento histórico e predito de aplicações. O Capítulo 5 apresenta as conclusões deste trabalho, dificuldades encontradas e propostas para trabalhos futuros. Finalmente, são apresentadas as referências. 


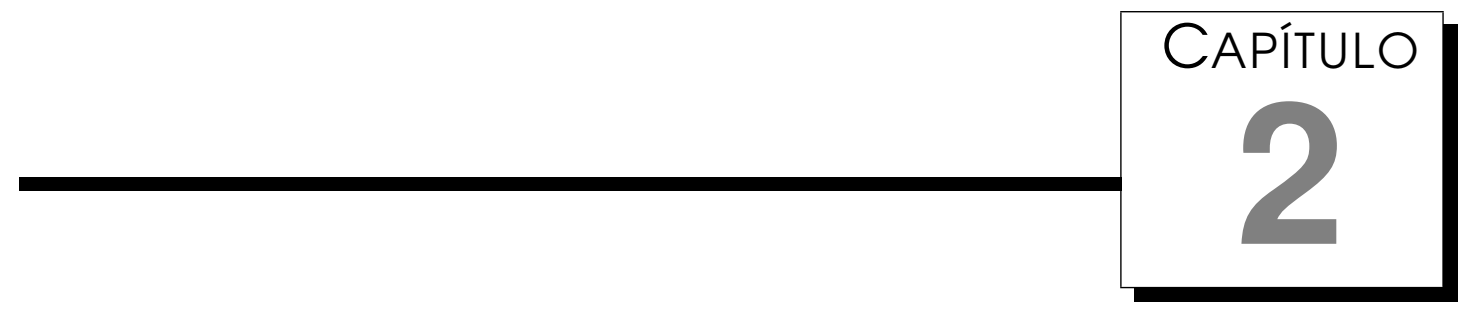

\section{Trabalhos Relacionados}

\section{1 Considerações Iniciais}

Este capítulo apresenta trabalhos relacionados que abordam o problema de otimização de acesso a dados distribuídos. Em seguida, são apresentados e discutidos trabalhos que realizam predição de comportamento de processos em ambientes computacionais.

\subsection{Abordagens para otimização de acesso a dados distribuídos}

Muitas pesquisas têm sido conduzidas com objetivo de aumentar o desempenho de acesso a dados em ambientes computacionais de larga escala. Esses trabalhos focam, principalmente, em questões de replicação, migração, distribuição e manutenção de consistência de dados.

Oliker et al. (2004) propõem uma abordagem estática de distribuição de dados e três algoritmos de escalonamento Sender-Initiated (SI), Receiver-Initiated (RI), Symmetrically-Initiated (SYI). Essa abordagem visa a otimização de desempenho global do sistema por meio da alocação de tarefas em regiões (sites) onde os dados estão disponíveis. Segundo os autores, bons resultados foram obtidos especialmente com a estratégia SI, a qual reduz o tempo de execução médio de aplicações em até $60 \%$ quando comparada a outras abordagens locais e, ainda, é capaz de executar $40 \%$ mais tarefas.

Sun e Xu (2004) propõem dois algoritmos de consistência de dados: Lazy-Copy (LC) e Aggressive-Copy (AC). LC atualiza réplicas somente quando 
necessário, ou seja, no instante em que um usuário faz uma requisição. Essa abordagem tende a reduzir o consumo de banda dos canais de comunicação, evitando transferências desnecessárias, isto é, quando dados são constantemente modificados e não requisitados. AC atualiza réplicas sempre que uma alteração ocorre no arquivo original. Além disso, AC fornece garantia total de consistência, enquanto LC, garantia parcial. Na comparação entre esses dois algoritmos, os autores observam que o AC reduz a latência de acesso a dados, enquanto o LC reduz o consumo de banda do sistema de comunicação.

Rahman et al. (2005) apresentam um modelo que utiliza uma abordagem de agrupamento de dados, K-Nearest Neighbor (KNN), para selecionar a réplica que apresenta menor custo para determinada aplicação. Para selecionar essa réplica, os autores desenvolveram uma técnica de otimização que considera as latências da rede e o estado das unidades de armazenamento (discos). Diferentes padrões de acesso a dados foram investigados e comparados e, segundo os autores, sua abordagem apresenta bom desempenho para padrões de acesso sequencial e aleatório unitário.

Wang et al. (2006) assumem que réplicas de dados podem ser reagrupadas e acessadas em paralelo. A partir dessa estratégia, minimiza-se o tempo de acesso por meio da sobreposição de requisições aos dados, permitindo aumento de vazão (throughput) dos dados. Para validar sua abordagem, os autores propõem um algoritmo denominado Multi-Source Data Transfer (MSDT), que considera uma rede de comunicação dinâmica e visa o balanceamento de carga de dados. Réplicas são criadas em momentos de ociosidade dos recursos computacionais. Deve-se observar que esse trabalho não explora claramente o assunto de manutenção de consistência de dados, deixando lacunas para o leitor. Resultados apontam um fator de speedup entre 2,72 3,06 na comparação do MSDT com uma outra técnica proposta em Feng e Humphrey (2004). Apesar desses resultados, poucas análises comparativas foram apresentadas no trabalho.

Oldfield e Kotz (2006) propõem um arcabouço (framework), denominado Armada, que lança aplicações e define como arquivos são distribuídos sobre um sistema. Esse arcabouço também fornece controle e mecanismos de acesso a dados. Para otimizar o acesso, o Armada constrói grafos de dependência, que modelam operações de processamento e acesso a dados. Em experimentos comparando aplicações reestruturadas pelo Armada a aplicações originais, aumentou-se a vazão de redes de longa distância em até $40 \%$.

Dang e Lim (2007) propõem uma estrutura em árvore para correlacionar dados a regiões de uma grade computacional visando a redução de 
transferências de arquivos sobre a rede. No instante que uma tarefa necessita de um arquivo, a abordagem busca por dados com alta correlação antes de solicitar uma nova transferência. Isso reduz custos de comunicação e aumenta o desempenho global das aplicações.

Elghirani et al. (2007) definem serviços de gerenciamento de dados para a replicação de arquivos em regiões (sites) de uma grade computacional. Os autores também propõem uma abordagem, baseada em busca Tabu, para o escalonamento de tarefas visando a otimização do tempo de resposta e utilização do sistema. A busca Tabu concentra-se em boas soluções para replicação de dados, considerando o tempo de acesso e processamento de dados. Resultados confirmam aumento no desempenho entre $8 \%$ e $35 \%$, dependendo da abordagem de replicação e algoritmo de escalonamento considerados.

Sato et al. (2009) propõem um algoritmo de replicação baseado em agrupamento de arquivos. O algoritmo agrupa arquivos de acordo com o relacionamento entre seus padrões de acesso. As réplicas dos arquivos são armazenadas nos dispositivos a fim de satisfazer os seguintes requisitos de acesso futuro: minimização de custos relativos à latência e maximização de capacidade de armazenamento. O problema de replicação é modelado como um problema de particionamento em grafos e programação inteira (Sato et al., 2008). Experimentos foram conduzidos a fim de comparar o algoritmo proposto a técnicas sem replicação e sem agrupamento. Resultados apontam que o algoritmo proposto auxilia no gerenciamento eficiente de réplicas.

Kim et al. (2009) propõem uma técnica que aumenta o desempenho de acesso a dados por meio da alocação em regiões mais próximas aos elementos de processamento solicitantes. Os autores consideram um cenário baseado em traços $^{1}$ sintéticos executados sobre o testbed ${ }^{2}$ PlanetLab para a avaliação de heurísticas. Resultados demonstram que a alocação de recursos é mais adequada que a oferecida por técnicas convencionais, tais como as baseadas em latência ou aleatórias.

Chervenak et al. (2009) propõem um arcabouço (framework) denominado Replica Location Service (RLS), o qual mantém e fornece informações de localização de réplicas de arquivos. RLS é utilizada por diversos ambientes de produção tais como Laser Interferometer Gravitational Wave Observatory (LIGO) (Abramovici et al., 1992), Earth System Grid (ESG) (National Center, 2005) e Pegasus (Deelman et al., 2003). Os autores demonstram que um servidor RLS individual tem desempenho satisfatório e suporta milhões de

\footnotetext{
${ }^{1}$ Termo utilizado em diversas áreas da computação, incluindo a de processamento de alto desempenho, que representa comportamentos de aplicações (Burtscher e Sam, 2005).

${ }^{2}$ Uma plataforma para condução de experimentos com fins de validação de teorias científicas, ferramentas computacionais ou novas tecnologias (Chun et al., 2003).
} 
requisições quando comparado a um servidor de banco de dados MySQL.

AL-Mistarihi e Yong (2009) propõem uma abordagem para solucionar o problema de seleção de réplicas mais adequadas para atender as operações de leitura e escrita de determinada aplicação. Nesse trabalho, os autores consideram o mecanismo Analytical Hierarchy Process (AHP) e uma extensão do simulador OptorSim (Bell et al., 2003). AHP é empregada para o tratamento desse problema de otimização, reduzindo uma abordagem multiobjetivo em um objetivo único. Deve-se ressaltar que os autores não comparam os resultados de sua abordagem à demais técnicas disponiveis no simulador OptorSim tais como Least Recently Used (LRU), Least Frequently Used (LFU) e Modelo Econômico.

\subsection{Predição do comportamento de processos}

Diversas pesquisas têm adotado técnicas estatísticas para analisar dados e construir modelos probabilísticos, tanto para a caracterização da carga de trabalho de ambientes distribuídos quanto para previsão de desempenho. Além disso, esses modelos podem ser empregados para auxiliar no diagnóstico de falhas, alocação de recursos e otimização de desempenho de sistemas.

Faerman et al. (1999) propõem um modelo regressivo, denominado Adaptive Regression Modeling (AdRM), para estimar instantes de transferência de arquivos em aplicações distribuídas. A fim de realizar experimentos, os autores utilizaram o Network Weather Service (NWS) para coletar o estado de canais de comunicação e recursos distribuídos, armazenando essas informações históricas para posterior análise. O modelo proposto foi avaliado considerando duas aplicações que manipulam grandes volumes de dados: Synthetic Aperture Radar Atlas (SARA) e Storage Resource Broker (SRB). A aplicação de aquisição de imagens SARA manipula arquivos pequenos (de 1 a $3 \mathrm{MB}$ ), enquanto SRB trabalha sobre arquivos maiores (16MB ou mais). Os autores conduziram experimentos comparativos entre seu modelo (AdRM) e o modelo Raw BandWidth (RBW). Resultados demonstram maior acurácia nas predições conduzidas pelo modelo AdRM. Uma das principais limitações do trabalho é a falta de comparação com demais modelos de estimação e predição de séries temporais.

Wang et al. (2004) empregam técnicas de aprendizado de máquina, baseadas em Classification And Regression Trees (CART), para modelar dispositivos de armazenamento. Os autores propõem duas abordagens para a predição de desempenho desses dispositivos em função da carga de trabalho: a primeira considera um modelo de predição por requisição e, a segunda, agrupa essas requisições, considerando uma média comportamental. 
Experimentos conduzidos sobre cargas de trabalho reais demonstram que os dois modelos apresentam erro médio igual a $17 \%$ e $38 \%$, respectivamente.

Liblit et al. (2005) propõem um algoritmo de depuração que isola erros (bugs) em código fonte. Os autores consideram um depurador estatístico para a análise dinâmica de código. Esse depurador monitora e examina o comportamento de um programa durante sua execução. A etapa de exame considera uma técnica de regressão logística, a fim de selecionar predicados e predizer erros de execução. Resultados demonstram que essa abordagem detecta tanto faltas esperadas quanto imprevistas, no entanto, para um número reduzido de programas.

$\mathrm{Xu}$ et al. (2009) propõem uma metodologia que examina logs de terminais com o objetivo de detectar falhas em tempo de execução. A partir desses logs, propriedades sobre o estado de execução do sistema são identificadas e, posteriormente, a técnica de aprendizado de máquina Principal Component Analysis (PCA) é empregada para detectar anomalias. Experimentos foram conduzidos utilizando o servidor de jogos on-line Darkstar e o sistema de arquivos distribuído Hadoop. Resultados para o servidor Darkstar indicaram ausência de anomalias em canais de comunicação. Isso sugere a investigação de outras propriedades a fim de detectar anomalias. No estudo de caso conduzido sobre o sistema Hadoop, os resultados auxiliaram operadores e desenvolvedores a identificar determinados tipos de comportamento anômalo, o que aumenta a eficiência na prevenção de falhas desse sistema.

Huaiming et al. (2008) propõem um modelo para a predição de predicados de consultas SQL a fim de realizar execução especulativa. Os parâmetros são ajustados de acordo com o estado dos recursos do sistema por meio de um algoritmo de predição adaptativo com dois níveis de escala. Para cada nível é proposta uma métrica, isto é, acurácia ou eficiência. Experimentos foram conduzidos no sistema DBroker, os quais demonstraram que o algoritmo proposto e o método de execução especulativa reduzem o tempo de resposta de consultas SQL.

\subsection{Análise dos trabalhos relacionados}

Esta seção indica possíveis lacunas nos trabalhos relacionados e apresenta alternativas para essas limitações, tanto na otimização de acesso a dados distribuídos, quanto na predição do comportamento de processos.

\subsection{Sobre as abordagens para otimização de acesso a dados}

Nota-se que todos os trabalhos previamente discutidos na Seção 2.2 não consideram o comportamento dinâmico de aplicações, nem estimações ou 
predições, a fim de tomar decisões de otimização.

Essa limitação motivou a proposta de uma heurística que emprega janela de eventos futuros de comprimento fixo, a fim de otimizar operações futuras de leitura e escrita sobre arquivos (Ishii e de Mello, 2009). Essa janela contém estimativas de operações futuras sobre dados, as quais são empregadas pela heurística a fim de tomar decisões sobre a replicação, migração e manutenção de consistência de dados.

Esse trabalho constatou que, ao estimar comportamento futuro de aplicações, pode-se aumentar o desempenho de processos em diferentes cenários. Por exemplo, ao considerar um ambiente com $100 \%$ de operações somente leitura, essa heurística é capaz de aumentar o desempenho médio em até $100 \%$. Entretanto, em ambientes com baixa frequência de operações de escrita ( $5 \%$ de operações de escrita e $95 \%$ leitura) ou baixa frequência de leituras ( $95 \%$ de operações de escrita e $5 \%$ leitura), a heurística não apresentou redução significativa no tempo médio de execução das aplicações.

Nesse trabalho prévio (Ishii e de Mello, 2009), o comprimento da janela de estimativas futuras de operações de leitura e escrita é fixo e atribuído pelo administrador do sistema. A possibilidade de configuração de diferentes comprimentos de janela e a análise de resultados experimentais motivaram estudos adicionais com objetivo de verificar se uma janela de eventos futuros, com operações do mesmo tipo, podem reduzir atrasos de acesso a dados e, consequentemente, otimizar o tempo médio de execução de aplicações. Essa investigação é detalhada na Seção 4.4, onde uma janela de eventos futuros adaptativa é proposta.

\subsubsection{Sobre a predição do comportamento de processos}

A principal limitação dos trabalhos relacionados, Seção 2.3 , está no fato de não avaliarem propriedades relativas ao conhecimento extraído de aplicações. Dentre tais propriedades estão a estocasticidade, linearidade e a estacionariedade. Ao considerar essas propriedades, obtém-se modelos com maior acurácia e, que, portanto, geram melhores resultados de predição do comportamentos de processos.

Essa limitação tem motivado o grupo de pesquisas BIOCOM ${ }^{3}$ do ICMC-USP, no qual esta tese se insere, a desenvolver pesquisas que envolvem a predição de comportamento de aplicações por meio da análise de séries temporais, sistemas dinâmicos e teoria do caos. Em um dos principais trabalhos do grupo, voltado para a detecção de comportamento, Mello e Yang (2009) demonstram e validam a importância de empregar o conhecimento sobre aplicações a fim de otimizar o escalonamento de processos. Além

\footnotetext{
${ }^{3}$ http://www.icmc.usp.br/ biocom
} 
disso, os autores confirmam que a comunicação entre processos apresenta comportamento não linear e instável, exigindo a adoção de técnicas mais sofisticadas, principalmente, baseadas em teoria do caos, para obter modelagens mais precisas.

Em outro trabalho do grupo, Dodonov e de Mello (2010) propõem uma abordagem para a predição de comportamento de processos por meio da avaliação de propriedades caóticas, bem como a detecção de estados críticos de execução de processos. Esse comportamento é representado por meio de séries temporais. Os autores ainda empregam a rede neural Self-Organizing Neural Network for Detecting Novelties (SONDE), proposta em (Albertini e de Mello, 2007), também vinculada ao BIOCOM, para agrupar comportamentos similares e, posteriormente, predizer o comportamento de processos de maneira on-line. Predições são utilizadas a fim de suportar decisões de escalonamento de processos. Um algoritmo de escalonamento foi proposto e experimentos foram conduzidos com objetivo de compará-lo a outros algoritmos. Resultados confirmam a redução do tempo de resposta em 10 vezes, quando comparado a outras técnicas comumente empregadas na literatura.

Essas iniciativas do grupo motivaram estudos adicionais sobre a análise de processos geradores de séries temporais (Capítulo 3), os quais são empregados nesta tese (Capítulo 4), a fim de obter modelos e predições de eventos de acesso a dados com maior acurácia.

\subsection{Considerações Finais}

Este capítulo apresentou trabalhos relacionados ao problema de acesso a dados, os quais visam, essencialmente, abordar questões relativas à replicação, migração, consistência de dados, estimativas de transferência e custos de acesso.

A investigação desses trabalhos e a identificação de lacunas nessa linha de pesquisa motivaram esta tese. Dentre os principais pontos negativos dos trabalhos descritos neste capítulo estão: o fato de que não consideram operações de escrita, empregam abordagens estáticas de otimização e, por fim, poucos oferecem algum mecanismo para compreensão, análise e predição de comportamento de processos a fim de otimizar operações de leitura e escrita em ambientes distribuídos.

Nesse sentido, estudos foram realizados a fim de considerar abordagens dinâmicas de otimização, e para compreender, analisar e predizer comportamentos de processos. Essa etapa motivou estudos adicionais sobre a análise de séries temporais, os quais são apresentados no capítulo seguinte. 


\section{$-3$ \\ Uma Metodologia para Classificação de Séries Temporais}

Este capítulo apresenta os principais conceitos de séries temporais (Seção 3.2) e propõe uma metodologia para a análise de séries, a qual é, posteriormente, aplicada na predição de operações de leitura e escrita em arquivos. Essas predições são consideradas na otimização de acesso a dados proposta nesta tese.

A Seção 3.3 discute técnicas de modelagem para predição. Em seguida, uma taxonomia para classificação de séries temporais é proposta na Seção 3.4. Essa taxonomia é utilizada para indicar técnicas de modelagem de acordo com as propriedades das séries temporais. A Seção 3.5 emprega testes de aderência para a classificação de séries temporais, seguindo a taxonomia proposta na Seção 3.4. Experimentos utilizando dados reais foram conduzidos, Seção 3.6, com objetivo de validar os testes de aderência definidos na Seção 3.5. Finalmente, a Seção 3.7 avalia se o modelo indicado pela metodologia proposta realmente fornece erro mínimo na predição de eventos de acesso a dados.

\section{1 Considerações Iniciais}

A análise das informações produzidas por sistemas reais tem atraído pesquisadores, que visam, principalmente, modelar e compreender suas características. Em geral, resultados produzidos por sistemas reais apresentam elevada dependência temporal, ou seja, há correlação parcial ou total entre observações adjacentes no tempo. 
A área responsável por avaliar e modelar tais dependências é denominada Análise de Séries Temporais (Morettin e Toloi, 2006). Essa área busca modelar séries temporais com o objetivo de extrair comportamentos embutidos nos dados, tais como tendência (observações podem ter comportamento ascendente ou descendente ao longo do tempo) e sazonalidade (observações podem ter comportamentos similares em diferentes intervalos de tempo).

$\mathrm{Na}$ modelagem de sistemas, observações (ou dados extraídos) são organizadas em função de suas variáveis. Assim, uma variável $X$ tem um conjunto de observações associado no formato $\left(x_{1}, x_{2}, \ldots, x_{n}\right)$, onde os índices são instantes de tempo. Por meio da modelagem dessas séries temporais pode-se interpretar seus comportamentos e, consequentemente, compreender suas propriedades. Essa abordagem permite o estudo de sistemas reais tais como o movimento de planetas, galáxias, ligações entre neurônios, mercado financeiro, epidemias, crescimento populacional, alterações climáticas, etc. (Marwan et al., 2007). Ao modelar um sistema, é possível predizer seu comportamento no tempo, o que auxilia a antecipar a tomada de decisões. No contexto deste trabalho, busca-se antecipar decisões a fim de otimizar o acesso a dados distribuídos.

As vantagens provenientes da modelagem de séries temporais motivam esta tese, entretanto, antes dessa etapa deve-se investigar os processos que deram origem a tais séries (processos geradores). Após a compreensão desses processos, pode-se selecionar o conjunto de técnicas mais adequadas (ou boas candidatas) para modelar séries temporais. Essa etapa, geralmente, requer um especialista e também informações detalhadas da origem da série, o que nem sempre está disponivel. Mesmo assim, ainda podem haver falhas devido à subjetividade imposta pelo especialista (Shumway e Stoffer, 2006). Somente após essa etapa, o modelo adotado pode ser aprimorado e corretamente avaliado.

A necessidade de um especialista e a falta de uma metodologia de análise de processos geradores motivaram, no contexto desta tese, a proposta de uma abordagem que aplica testes de aderência em séries temporais. Esses testes permitem a classificação de processos geradores e auxiliam na escolha de técnicas mais adequada para a modelagem de séries temporais.

Com o uso dessa abordagem, evita-se a adoção de técnicas pouco representativas e pode-se obter conclusões mais refinadas a respeito das propriedades de séries temporais. Essa abordagem ainda auxilia na automatização do processo de seleção da melhor técnica, sem a intervenção de um especialista. Resultados confirmam que há aumento na acurácia de modelagem (Seção 3.7). 


\subsection{Revisão de séries temporais}

Séries temporais são utilizadas para organizar e representar observações coletadas ao longo do tempo. Ao modelar essas séries, pode-se investigar, analisar e predizer comportamentos. Séries temporais são constituídas por observações sequenciais coletadas de uma (univariada) ou mais variáveis (multivariadas) ordenadas no tempo.

As séries univariadas são compostas por uma única variável $X$, cujas observações podem ser representadas no formato $\left(x_{1}, x_{2}, \ldots, x_{n}\right)$, considerando o intervalo de tempo $t \in[1, n]$, onde $n$ é o número de observações. No entanto, em séries multivariadas, $k$ variáveis são observadas em cada instante de tempo $t$. Essas séries podem ser descritas no formato $\left(x_{1 t}, x_{2 t}, \ldots, x_{k t}\right)$, onde $t \in[1, n]$.

A Figura 3.1 apresenta um exemplo de série temporal, onde $X_{t}$ é uma variável aleatória definida sobre um espaço amostral $\Omega$, e portanto, é uma função de duas variáveis, $X(t, \omega), t \in T, \omega \in \Omega$, onde $T$ é um conjunto que pode assumir valores tanto em $\mathbb{Z}$ quanto em $\mathbb{R}$. Para um dado $\omega$ fixo, tem-se uma função densidade de probabilidade (f.d.p.) em $t$, que pode ser diferente para quaisquer $t_{i}$ e $t_{j}, \forall t_{i} \neq t_{j}$.

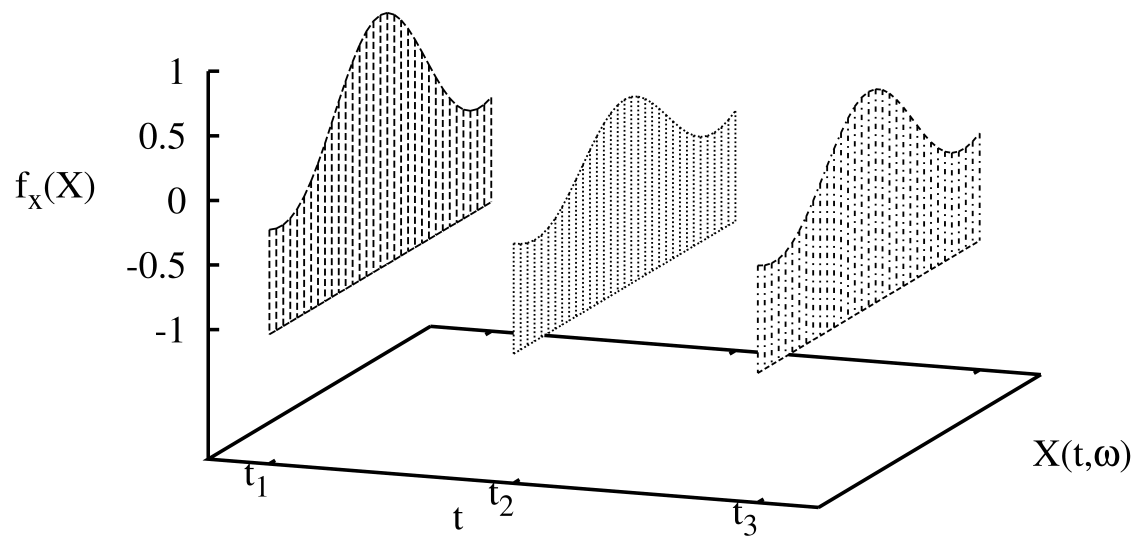

Figura 3.1: Representação de uma série temporal. Para cada instante de tempo $t$, tem-se uma variável aleatória $X(t, \omega)$, com uma f.d.p. $f_{x}(X)$ (Morettin e Toloi, 2006).

Além da abordagem intuitiva previamente apresentada, pode-se definir, formalmente, uma série temporal $X_{t}$ pela soma de três componentes não observáveis, $X_{t}=\Gamma_{t}+S_{t}+\varepsilon_{t}$, onde $\Gamma_{t}$ representa tendência, $S_{t}$ sazonalidade e $\varepsilon_{t}$ um componente aleatório (Morettin e Toloi, 2006). A componente $\Gamma_{t}$, 
ou tendência, representa as variações no comportamento da série. $S_{t}$, ou sazonalidade, indica se um determinado comportamento da série tende a se repetir em intervalos de tempo $\Delta t$. Esses componentes são denominados não observáveis pois não são coletados, mas sim inferidos por meio de relações entre observações no tempo (Morettin e Toloi, 2006).

O estudo de séries temporais envolve a investigação de seus processos geradores. Ao reconhecer o processo gerador de uma série é possível inferir a melhor técnica para sua modelagem. Existem três aspectos importantes a serem considerados nessa investigação: a estocasticidade, a linearidade e a estacionariedade.

O primeiro avalia se uma série temporal é determinística ou estocástica. Uma série determinística tem comportamento recorrente, ou seja, ela repete um dado conjunto de observações no tempo, em escala similar ou em diferentes proporções (Box et al., 1994). Sistemas que apresentam essas características podem ser modelados por meio de equações diferenciais determinísticas. Assim, a modelagem e a predição da próxima observação baseia-se no comportamento das observações passadas. Entretanto, mesmo sendo determinístico, alguns desses sistemas são muito sensíveis às condições iniciais e a pertubações mínimas, o que dificulta predições de longo prazo. No entanto, mesmo para sistemas caóticos, predições de curto prazo são viáveis (Marwan et al., 2007).

Por outro lado, séries temporais também podem ser geradas a partir de sistemas estocásticos, onde observações futuras dependem do estado anterior (assim como nos sistemas determinísticos) e, também, de componentes aleatórios. Técnicas estatísticas são geralmente utilizadas para modelagem dessas séries, uma vez que representam observações dependentes no tempo e termos probabilísticos (Morettin e Toloi, 2006).

A investigação de componentes determinísticos e estocásticos em séries temporais auxilia na escolha da melhor técnica de modelagem. Pesquisadores têm proposto e aplicado diferentes técnicas para avaliar esses componentes. Marwan et al. (2007); Marwan (2008) apresentam como a ferramenta Recurrence Plot (RP) pode ser utilizada no estudo desses componentes. Esses trabalhos também apresentam aplicações em ciências biológicas, variações cambiais, detecção de falhas em sistemas mecânicos entre outros. Golestani et al. (2009) propõem uma abordagem que avalia determinismo e estocasticidade em séries por meio de seções de Poincaré e dimensão fractal de Higuchi. Esse método busca distinguir sinais determinísticos de aleatórios. Rao (2007) apresenta um estudo que aplica testes de raízes unitárias e técnicas de integração a fim de avaliar séries temporais, bem como fornecer métricas referenciais que suportem a tomada de decisões na seleção 
de modelos estocásticos ou determinísticos.

Após caracterizar um sistema em determinístico ou estocástico, é necessário focar em quais técnicas são mais eficazes para sua modelagem. No caso de um sistema determinístico, pode-se considerar abordagens baseadas em teoria do caos. Para sistemas estocásticos, técnicas estatísticas apresentam melhores resultados.

Além disso, outras propriedades do sistema necessitam ser investigadas, tais como a linearidade e a estacionariedade. Em séries temporais lineares, observações são organizadas por uma combinação linear entre valores passados e ruídos ${ }^{1}$. Assim, a linearidade é descoberta através do modelo, mapa ou processo que gerou a série. Em séries não lineares, as observações são obtidas por combinações não lineares entre ocorrências passadas e ruídos. Séries não lineares são mais difíceis de estudar e modelar.

Hegger et al. (1998) descrevem técnicas que avaliam a linearidade em séries temporais utilizando ferramentas disponiveis no pacote $\mathrm{Ti} \mathrm{sean}^{2}$. Esse pacote implementa o método surrogate data, que, baseado na série original, gera séries substitutas com objetivo de compará-las. As séries substitutas são, tipicamente, criadas a partir de um processo linear Gaussiano estacionário. Após essa etapa, um teste de hipótese é formulado, onde $H_{0}$ (hipótese nula) assume que não há diferença significativa entre o conjunto de séries amostrais e a original e, portanto, a série é linear. A hipótese alternativa $H_{a}$ pode ser aceita caso haja diferença significativa e, assim, a série original é dita não linear. Galka e Ozaki (2001) investigam e comparam diferentes técnicas para a avaliação de linearidade em séries temporais. Eles consideram séries amostrais (através de surrogate data) e avaliam as hipóteses por meio dos seguintes testes: irreversibilidade (Diks et al., 1995), preditor constante local (Schreiber e Schmitz, 1997) e um método proposto pelos próprios autores. Eles demonstram que ao aplicar fortes ruídos em séries temporais de grandes dimensões, o desempenho dos testes dependem da quantidade de ruídos e de suas propriedades espectrais.

White (1989) investiga uma rede neural com retroalimentação e verifica que existe pouco relacionamento entre suas entradas e saídas. Isso porque as entradas não contêm todas as variáveis que influenciam nas saídas. Assim, White observa que a falta de influência gera dois tipos básicos de erros: os inevitáveis, devido a relacionamentos inexatos entre entradas e saída; e os erros de aproximação, potencialmente, evitáveis. Dessa maneira, é proposto um teste de hipótese que verifica se erros de aproximação não ocorrem. Portanto, esse trabalho permite avaliar se a rede neural proposta é capaz de modelar exatamente um sistema sujeito a ruídos, ou se há alguma

\footnotetext{
${ }^{1}$ Podem ser definidos como valores inesperados ou aleatórios.

${ }^{2}$ http://www.mpipks-dresden.mpg.de/ tisean/Tisean_3.0.1/index.html
} 
estrutura negligenciada pela rede, o que caracteriza erros de aproximação potencialmente evitáveis.

Lee et al. (1993) comparam a rede neural multicamada proposta por White (1989) com outros testes que também identificam linearidade em séries: teste de Keenan, teste Tsay, teste White com matriz de informações dinâmicas, teste McLeod-Li, teste Ramsey Reset, teste Brock-Dechert-Scheinkman e teste Bispectrum. Resultados experimentais confirmam que nenhum teste foi o melhor entre todos. No entanto, o teste que aplica a rede neural multicamadas tem desempenho similar a todos os testes e, em alguns cenários, seu desempenho foi superior.

Após a investigação da linearidade em séries temporais, é necessário avaliar a estacionariedade. As observações em séries estacionárias são formadas por um estado particular de equilíbrio estatístico (Box et al., 1994), ou seja, elas evoluem aleatoriamente no tempo em torno de uma média e variância constantes (Morettin e Toloi, 2006).

As propriedades de séries estacionárias não são afetadas por mudanças no tempo de observação original, isto é, a função densidade de probabilidade (f.d.p.) associada com $n$ observações $\left(x_{1}, x_{2}, \ldots, x_{n}\right)$ não se altera mesmo após um deslocamento no tempo, dado por uma constante $k,\left(x_{1+k}, x_{2+k}, \ldots, x_{n+k}\right)$ (Box et al., 1994). Além disso, séries estocásticas $\left\{X_{t}, t \in \mathbb{N}\right\}$ são também denominadas fracamente estacionárias ou de segunda ordem, sempre que a média e a variância são constantes (ou seja, $E\left(X_{t}=\mu\right.$ ) e $\operatorname{var}\left(X_{t}\right)=\sigma^{2}$, respectivamente) (Morettin e Toloi, 2006).

Séries temporais que não satisfazem as condições de estacionariedade são denominadas não estacionárias. Essas séries apresentam geralmente comportamento evolutivo, principalmente, devido às variações da componente tendência e por modelarem processos explosivos (Morettin e Toloi, 2006). Em tais processos, a média de valores observados tende a aumentar ou diminuir quando novas observações são consideradas nos instantes de tempo subjacentes. Como um exemplo de processo com características evolutivas, considere um sistema que modela o crescimento de uma população, na forma $x_{t}=k \cdot x_{t-1}+\varepsilon_{t}$. Nesse modelo, $x_{t}$ representa o tamanho da população no instante $t, \varepsilon_{t}$ é um componente aleatório e $k$, uma constante que determina a tendência de evolução do tamanho da população ao longo do tempo. Uma abordagem que lida com tendência, nessa classe de séries temporais, são filtros lineares, os quais convertem a série $x_{t}$ em outra $y_{t}$. Assim, pode-se definir $z_{t}=x_{t}-y_{t}$, ou seja, uma série livre de tendências (Morettin e Toloi, 2006).

Ao classificar séries quanto à estacionariedade dos processos geradores, tem-se informações suficientes para a indicação da técnica mais adequada 
(ou grupo de técnicas) para modelagem. No entanto, ao negligenciar a presença de não estacionariedade, erros podem ser obtidos no cálculo de métricas relacionadas a sistemas dinâmicos, tais como expoente de Lyapunov, dimensão de correlação e entropias (Yu et al., 1998).

Duas técnicas são frequentemente empregadas na avaliação de estacionariedade: função de autocorrelação e separação espaço-tempo. A função de autocorrelação relaciona uma série consigo mesma, avaliando a similaridade entre as observações em função do tempo de separação entre elas. Essa função suporta a descoberta de padrões recorrentes. A separação espaço-tempo, proposta por Provanzale et al. (1992), atua de maneira similar com o objetivo de identificar correlações temporais nas séries. Ambas abordagens podem ser utilizadas para avaliar como padrões se repetem ao longo do tempo. Variações em escala (ou amplitude) nessas avaliações indicam que as séries integram observações passadas para gerar outras futuras, o que caracteriza não estacionariedade.

Li et al. (2009) utilizam a função de autocorrelação a fim de avaliar a estacionariedade do tráfego de redes de longa distância e, portanto, melhorar a análise de séries. Provanzale et al. (1992) empregam a separação espaço-tempo para avaliar e distinguir a existência de comportamentos aleatórios ou caóticos de baixa dimensionalidade em séries temporais. Yu et al. (1998) estenderam o trabalho de Provanzale et al. (1992), propondo uma indexação espaço-tempo, onde funções de densidade das observações são normalizadas de acordo com índices no tempo. Esses trabalhos visam aumentar a acurácia da modelagem de séries temporais por meio da avaliação de seus graus de estacionariedade.

\subsection{Técnicas de Modelagem}

Nesta seção, as principais técnicas de modelagem são apresentadas. O objetivo de todas elas é compor uma estratégia que represente, com erro reduzido, comportamentos de séries temporais. No contexto desta tese, aplica-se o modelo obtido na predição de operações de leitura e escrita em arquivos.

\subsection{Modelagem baseada em ferramentas determinísticas}

A transformação das operações de leitura e escrita em séries temporais permite investigar suas variações de comportamento no tempo. Para compreender essas variações, propriedades de sistemas dinâmicos e teoria do caos podem auxiliar na modelagem e análise dessas séries. Um sistema dinâmico é composto por um conjunto de estados e uma regra que determina 
seu estado atual em função do passado.

Ao modelar o conhecimento embutido em um sistema dinâmico compreende-se a repetição de seus padrões, o que permite investigar suas tendências ou realizar agrupamento, classificação e predição. Uma abordagem para isso é a reconstrução ou transformação das saídas produzidas por sistemas dinâmicos, representadas por séries temporais unidimensionais, em um conjunto de estados (pontos) em espaço Euclidiano de $m$ dimensões. Segundo o teorema de imersão de Takens (Takens, 1981), uma série temporal $x_{1}, x_{2}, \ldots, x_{n}$ pode ser reconstruída em espaço multidimensional $x_{n}(m, \tau)=$ $\left(x_{n}, x_{n+\tau}, \ldots, x_{n+(m-1) \tau}\right)$, onde $m$ é a dimensão embutida e $\tau$ representa a dimensão de separação (ou time delay).

Após a obtenção de ambas dimensões, uma série pode ser reconstruída em seu espaço fase. Esse espaço considera somente relações entre estados (observações ou conjuntos de observações). O componente tempo é removido e, portanto, regressões podem ser realizadas a fim de compreender tendências (de Mello, 2009).

A reconstrução no espaço fase pode ser conduzida por diferentes técnicas, nesta tese foram consideradas as técnicas False Nearest Neighbors (FNN) (Kennel et al., 1992) e Auto-Mutual Information (AMI) (Fraser e Swinney, 1986) para obtenção das dimensões embutida e separação, respectivamente.

A técnica FNN proposta por Kennel et al. (1992) calcula os vizinhos mais próximos de cada ponto (ou estado), no espaço de coordenadas de atraso (inicialmente com dimensão embutida igual a 1). Em seguida, uma nova dimensão é adicionada e a distância entre vizinhos mais próximos é novamente calculada. Caso a distância seja maior, os pontos são considerados falsos vizinhos, o que caracteriza a necessidade de mais dimensões para reconstruir o comportamento da série.

Kennel et al. (1992) consideram uma dimensão embutida $d$, onde o $r$-ésimo vizinho mais próximo de $y(n)$ é dado por $y^{(r)}(n)$. A distância Euclidiana entre o ponto $y(n)$ e seu $r$-ésimo vizinho mais próximo é definida na Equação 3.1. Ao adicionar uma nova dimensão, reconstrói-se a série em $d+1$ e adiciona-se a coordenada $(d+1)$ em cada vetor $y(n)$, que é incluída no cálculo da distância, termo $x(n+d T)$ da Equação 3.2. Dessa forma, é obtida a variação de distância ao adicionar uma nova dimensão, descrita na Equação 3.3.

$$
\begin{gathered}
R_{d}^{2}(n, r)=\sum_{k=0}^{d-1}\left(x(n+k T)-x^{(r)}(n+k T)\right)^{2} \\
R_{d+1}^{2}(n, r)=R_{d}^{2}(n, r)+\left(x(n+d T)-x^{(r)}(n+d T)\right)^{2}
\end{gathered}
$$




$$
V_{n, r}=\sqrt{\frac{R_{d+1}^{2}(n, r)-R_{d}^{2}(n, r)}{R_{d}^{2}(n, r)}}=\frac{\left|x(n+d T)-x^{(r)}(n+d T)\right|}{R_{d}^{2}(n, r)}
$$

Se $V_{n, r}>R_{\mathrm{tol}}$, os pontos são considerados falsos vizinhos, onde $R_{\mathrm{tol}}$ é um limiar. Os autores concluem, empiricamente, que $R_{\text {tol }} \geq 10$ é um bom limite para o cálculo do FNN (Kennel et al., 1992).

Fraser e Swinney (1986) propõem a técnica de autoinformação mútua (AMI) para o cálculo da dimensão de separação. Para obtenção da dimensão de separação de uma série emprega-se AMI considerando diferentes deslocamentos no tempo. Em seguida, traça-se uma curva em função dos deslocamentos (iniciando em 1 e incrementando), adotando-se o primeiro mínimo como dimensão de separação.

A informação mútua média entre duas variáveis aleatórias é definida na Equação 3.4, onde $X$ e $Y$ seguem, respectivamente, as funções de probabilidades $P_{X}$ e $P_{Y}, X$ e $Y$ ocorrem em pares com distribuição conjunta $P_{X Y}$ (Kraskov et al., 2003).

$$
I(X ; Y)=\int P_{X Y}(x, y) \log _{2} \frac{P_{X Y}(x, y)}{P_{X}(x) P_{Y}(y)} \mathrm{d} x \mathrm{~d} y
$$

Após a obtenção das dimensões de separação e embutida diversas técnicas podem ser adotadas para modelar, adequadamente, uma série temporal. Dentre essas técnicas, três foram avaliadas nesta tese: RBF, preditor polinomial e predict. As próximas seções descrevem cada uma delas, que estão disponíveis no pacote Tisean (Hegger et al., 1998).

\section{Funções de Base Radial - RBF}

Radial Basis Function (RBF) é uma abordagem matemática para aproximar funções em espaço multidimensional. O objetivo é encontrar uma superfície que forneça ajuste adequado para as observações de uma série. Esse ajuste pode ser avaliado por medidas resumo, tais como o erro quadrático e erro absoluto (Haykin, 2009).

Essa abordagem emprega funções de base radial (geralmente Gaussianas ou Multiquadráticas, na Equação 3.5 a função $\varphi$ pode ser representada por $\varphi_{i}(x)=\exp ^{-\left\|x-c_{i}\right\|^{2}}, x_{1}$ e $x_{2}$ representam atributos do padrão de entrada $x$ ) para ativar resultados de saída em função de entradas (observações). Esses resultados de saída dependem do produto das $N$ funções radiais $\varphi_{i}$, centradas em $c_{i}$, pelos coeficientes de peso $w_{i}$ e bias $b$ (Haykin, 2009).

$$
F(x)=\sum_{i=1}^{N} w_{i} \varphi\left(\left\|x-c_{i}\right\|\right)+b
$$


Há duas etapas para utilizar o modelo RBF: treinamento e execução. A etapa de treinamento é utilizada para representar as observações da série de entrada na forma de funções radiais $\varphi_{i}$. Ainda nessa etapa, os coeficientes de peso $w_{i}$ são adaptados a fim de reduzir erros de representação da série de entrada. A implementação RBF disponivel no pacote Tisean fixa os centros das funções radiais por meio de uma avaliação prévia da série de entrada. Na fase de execução, a RBF é utilizada para predizer novas observações em função do comportamento modelado da série de entrada. Neste trabalho, a abordagem RBF é empregada na predição do comportamento de processos (operações de leitura e escrita).

\section{Preditor Polinomial}

Nessa abordagem, busca-se predizer eventos futuros de séries temporais por meio da aproximação de funções. A função $\pi_{i} f_{N}: \mathbb{R}^{m} \rightarrow \mathbb{R}, i=1, \ldots, m$ é escolhida como função base. Nesse caso, ela representa um preditor polinomial de $m$ variáveis de grau $d$. Os parâmetros livres $\left(\begin{array}{c}m+d \\ m\end{array}\right) \equiv \frac{(m+d) !}{m ! d !}$ do polinômio são obtidos a fim de minimizar a Equação 3.6 (Casdagli, 1989).

$$
\sum_{n=1}^{N-1}\left(\pi_{i} x_{n+1}-\pi_{i} f_{N}\left(x_{n}\right)\right)^{2}
$$

A Equação 3.6 representa um problema linear de mínimos quadrados que pode ser resolvido eficientemente por um algoritmo $\mathrm{QR}^{3}$ (Wang e Gragg, 2003). Portanto, tem-se um preditor polinomial adequado para uma dado grau $d$. O preditor resultante $f_{N}$ é uma forma padrão e conceitualmente simples de aproximação de funções. Além disso, $f_{N}$ tem garantia de convergência à medida que $N$ e $d$ aumentam, por intermédio do teorema de aproximação de Weierstrass ${ }^{4}$.

Uma limitação dessa abordagem é a grande quantidade de parâmetros a serem definidos. Além disso, não há ordem de convergência para $m>1$ e polinômios de alto grau apresentam tendência de oscilação indesejável e descontrolada.

\section{Predict}

A técnica predict utiliza predição linear para calcular médias de todas observações (pontos ou estados) no espaço fase. Essa abordagem extrai

\footnotetext{
${ }^{3}$ Para qualquer matriz $A$ (de ordem $n$ ), existe uma matriz unitária $Q$, tal que $A=Q R$, onde $R$ é uma matriz triangular superior com elementos reais e não negativos (Francis, 1961).

${ }^{4}$ Seja $f$ uma função real contínua em $[a, b]$ e para qualquer $\varepsilon>0$, há um polinômio $p$ em $[a, b]$ tal que, $|f(x)-P(x)|<\varepsilon$, para todo $x \in[a, b]$. Ou seja, qualquer função contínua em um intervalo fechado e limitado pode ser uniformemente aproximada naquele intervalo através de polinômios em quaisquer graus de precisão (Jeffreys e Jeffreys, 1988)
} 
correlações entre estados, o que permite explorar a previsibilidade da série temporal.

Essa abordagem aproxima o comportamento dinâmico local por meio de uma constante. Em um espaço fase, sempre que se desejar predizer observações para $n+k$ instantes futuros, todos os vizinhos de $s_{n}$ devem estar disponíveis. A predição é definida na Equação 3.7, onde $V$ é a vizinhança e $k$ é o número de pontos a serem preditos. Essa equação representa uma média das observações vizinhas de um determinado estado do sistema.

$$
\hat{s}_{n+k}=\frac{1}{\left|V_{n}\right|} \sum_{s_{j} \in V_{n}} s_{j+k}
$$

\subsubsection{Modelagem baseada em ferramentas estocásticas}

A fim de compreender o processo de modelagem e análise de séries temporais através de ferramentas estocásticas, considere uma série temporal $\left\{X_{t}\right\}$, composta apenas por uma sequência de ruídos $\left(x_{t}=\varepsilon_{t}\right)$. Essa série é, portanto, denominada puramente aleatória (Purely Random Process) ou de ruído branco. Tal série pode ser modelada como um processo estacionário, onde seus ruídos são mutuamente independentes e identicamente distribuídos, cuja média é zero, $E(\varepsilon)=0$, e a variância é constante, $E\left(\varepsilon^{2}\right)=\sigma^{2}$.

No entanto, se as observações de uma série temporal $\left\{X_{t}\right\}$ são definidas pela soma entre um valor passado e um ruído branco, $x_{t}=x_{t-1}+\varepsilon_{t}$, seu processo é denominado passeio aleatório (Random Walk). Essa série pode ser modelada por um processo discreto e puramente aleatório com média $\mu$ e variância $\sigma^{2}$. Nessa situação, um valor futuro depende tanto de uma observação passada quanto de um valor aleatório. Portanto, essa série não é considerada um processo estacionário, devido à mudança na média $E\left(X_{t}\right)=t \cdot \mu$ e na variância $\operatorname{Var}\left(X_{t}\right)=t \cdot \sigma^{2}$ no tempo $t$.

\section{Modelo AR}

Dadas as ocorrências de uma série temporal $\left\{X_{t}\right\}$, definidas com base em $p$ valores de observações passadas, essa série pode ser modelada por um processo autorregressivo (Autoregressive Processes) de ordem $p, \operatorname{AR}(p)$. Esse modelo é definido por $x_{t}=c+\phi_{1} \cdot x_{t-1}+\phi_{2} \cdot x_{t-2}+\cdots+\phi_{p} \cdot x_{t-p}+\varepsilon_{t}$, tal que $\left\{\varepsilon_{t}\right\}$ é um processo puramente aleatório com média $E(X)=0$ e variância $\operatorname{Var}(X)=\sigma^{2}$.

Um processo autorregressivo de primeira ordem, $\operatorname{AR}(1)$, também denominado processo de Markov, é denotado por $x_{t}=c+\phi_{1} \cdot x_{t-1}+\varepsilon_{t}$. Entretanto, um processo autorregressivo pode ser generalizado para um processo de ordem $p, \operatorname{AR}(p)$, pelo emprego da equação $x_{t}=c+\sum_{i=1}^{p} \phi_{i} x_{t-i}+\varepsilon_{t}$. Sejam 
as raízes delimitadas no círculo de raio unitário, $|\phi| \leq 1$, o processo AR é estacionário para $X_{t}$.

O modelo autorregressivo busca a modelagem de séries e a predição de realizações futuras em função de suas observações passadas. Esse modelo adota valores correntes $\left(x_{t}\right)$ de séries relativos a observações passadas $x_{t-1}, x_{t-2}, \cdots, x_{t-p}$, onde $p$ determina o lag (ou atraso), que é utilizado na representação de relações entre a quantidade de observações passadas (Shumway e Stoffer, 2006). Esse modelo é comumente empregado na representação de fenômenos naturais, devido às relações de recorrência e influência entre observações.

\section{Modelo MA}

Seja uma série temporal $\left\{X_{t}\right\}$ e suas observações definidas com base em $q$ ruídos passados. Essa série pode ser modelada por um processo de Média Móvel (Moving Average Processes) de ordem $q, \operatorname{MA}(q)$, definido por $x_{t}=c+$ $\theta_{0} \cdot \varepsilon_{t}+\theta_{1} \cdot \varepsilon_{t-1}+\cdots+\theta_{q} \cdot \varepsilon_{t-q}$, tal que $\left\{\theta_{q}\right\}$ são as raízes do modelo, $c$ é uma constante e $\left\{\varepsilon_{t}\right\}$, um processo puramente aleatório com média $E(X)=0$ e variância $\operatorname{Var}(X)=\sigma^{2}$. Um processo modelado por $\operatorname{MA}(q)$ é estacionário, pois tem média e variância constante no tempo. O termo média móvel origina-se do fato que $x_{t}$ pode ser calculado em função da média e da soma ponderada de observações passadas de $\varepsilon$ (Hamilton, 1994; Morettin e Toloi, 2006).

Modelos de Média Móvel são geralmente utilizados nas áreas econômicas e financeiras, nas quais indicadores são diretamente afetados por variáveis aleatórias, tais como decisões governamentais e falta de matérias-primas. Essas variáveis nem sempre influenciam imediatamente o valor de um produto, porém geram uma variação em observações subsequentes.

\section{Modelo ARMA}

Uma série temporal $\left\{X_{t}\right\}$ pode, ainda, ser modelada segundo $p$ observações e $q$ ruídos passados. Nesse caso, a área estatística sugere utilizar o modelo Autoregressive and Moving Average (ARMA), que combina os modelos autorregressivo e de média móvel, conforme é demonstrado na Equação 3.8.

$$
x_{t}=c+\phi_{1} x_{t-1}+\phi_{2} x_{t-2}+\ldots+\phi_{p} x_{t-p}+\varepsilon_{t}+\theta_{1} \varepsilon_{t-1}+\theta_{2} \varepsilon_{t-2}+\ldots+\theta_{q} \varepsilon_{t-q}
$$

Esse modelo é formalmente definido por $\operatorname{ARMA}(p, q)$, onde $p$ representa a ordem da parte autorregressiva, $\operatorname{AR}(p)$, e $q$ refere-se à ordem da média móvel, $\operatorname{MA}(q)$. De maneira geral, o modelo autorregressivo permite relacionar valores futuros com informações históricas, enquanto a média móvel mensura erros 
na análise de valores históricos. A relevância do modelo ARMA consiste em sua habilidade de modelar uma série temporal estacionária com menos parâmetros que a aplicação isolada de um modelo puramente autorregressivo e de média móvel (Chatfield, 2004).

\section{Modelo ARIMA}

ARMA modela séries temporais estacionárias. Contudo, na prática, grande parte das séries temporais reais não são estacionárias. Nesse sentido, uma generalização do modelo ARMA, a fim de modelar séries temporais não estacionárias, é definida pelo modelo ARIMA (modelo ARMA integrado).

No modelo ARIMA, uma série temporal não estacionária é inicialmente integrada, a fim de remover a fonte não estacionária de variação, tornando-a estacionária. Formalmente, o componente $x_{t}$ do modelo ARMA, Equação 3.8, é substituído por $w_{t}$, ou seja, se $w_{t}$ é uma diferença de $x_{t}$, então $x_{t}$ é uma integral de $w_{t}$. O modelo ARIMA é dito ser integrado, o que permite ajustes no modelo estacionário para que observações distintas sejam integradas (somadas), visando um modelo para séries originalmente não estacionárias (Equação 3.9).

$$
x_{t}=c+\phi_{1} w_{t-1}+\phi_{2} w_{t-2}+\ldots+\phi_{p} w_{t-p}+\varepsilon_{t}+\theta_{1} \varepsilon_{t-1}+\theta_{2} \varepsilon_{t-2}+\ldots+\theta_{q} \varepsilon_{t-q}
$$

\subsection{Classificação dos processos geradores de séries temporais}

Após apresentar conceitos de séries temporais e trabalhos relacionados a técnicas de análise, esta seção organiza ferramentas de modelagem de acordo com uma estrutura em árvore (Figura 3.2), e, ainda, discute as principais abordagens para auxiliar na modelagem de séries.

No primeiro nível da Figura 3.2, tem-se uma série temporal qualquer. No segundo nível, a série pode ser classificada em determinística ou estocástica. Sempre que a série é determinística, ela pode ser avaliada por meio de ferramentas de sistemas dinâmicos e de teoria do caos. Nesse ramo da árvore, considera-se a técnica FNN (Kennel et al., 1992) para o cálculo da dimensão embutida e AMI para o cálculo da dimensão de separação (Fraser e Swinney, 1986), isto é, o atraso entre observações consecutivas. Uma vez obtidas ambas dimensões, a série pode ser reconstruída em seu espaço fase (Marwan et al., 2007). O espaço fase considera somente estados (observações ou conjunto de observações) que levam a outro estado futuro. O componente 


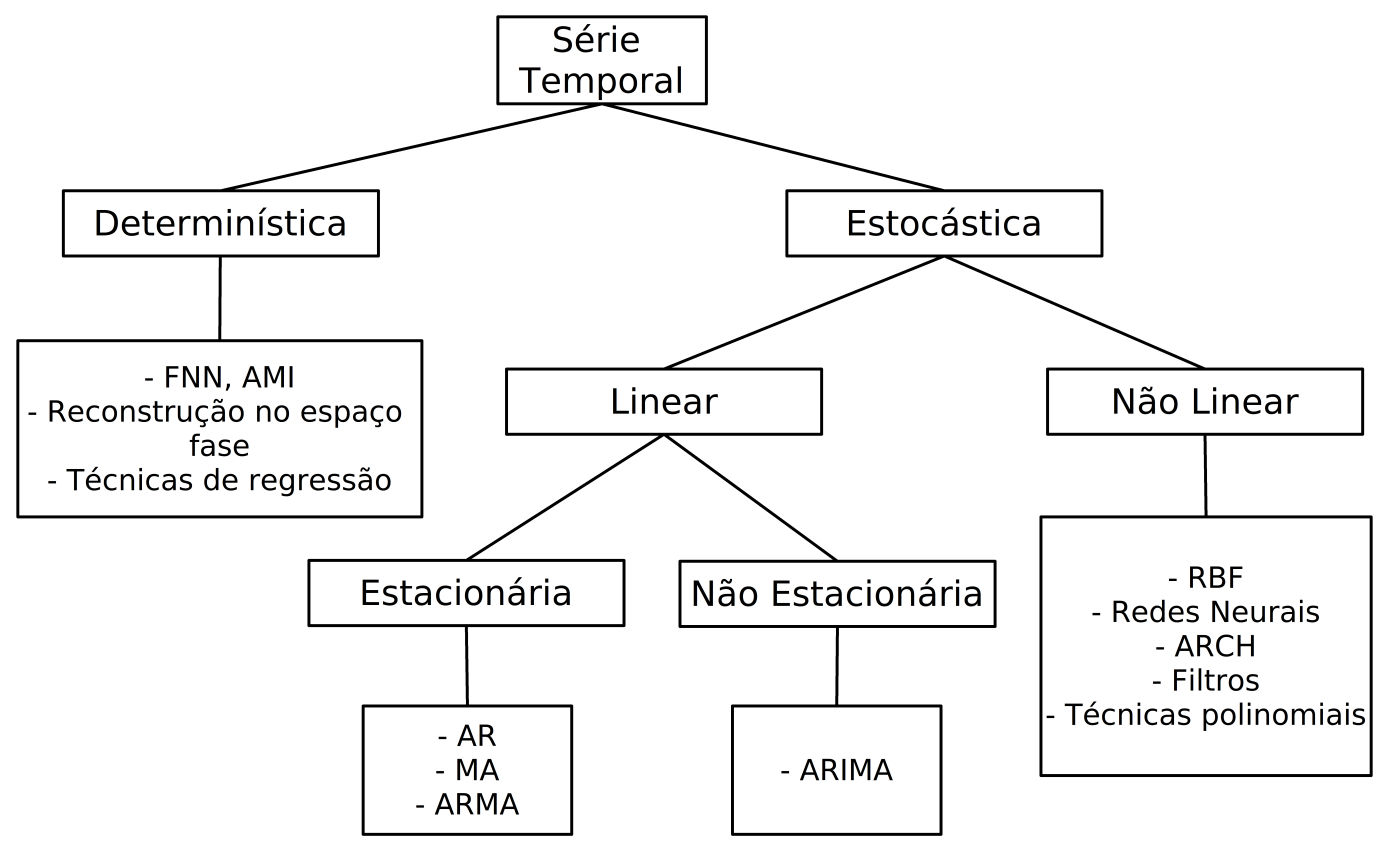

Figura 3.2: Uma taxonomia que relaciona os principais critérios considerados na análise de séries temporais, bem como as principais ferramentas que podem ser utilizadas na modelagem e avaliação.

tempo é removido do espaço fase, portanto, regressões podem realizadas sob tais estados a fim de compreender tendências.

Ainda no segundo nível da árvore existem as séries estocásticas. Nessa categoria, são necessárias avaliações adicionais com objetivo de selecionar a melhor técnica de modelagem. Nesse sentido, o terceiro nível, avalia graus de linearidade. Séries não lineares podem ser tratadas por meio de modelos não lineares tais como funções de aproximação de base radial (RBF), redes neurais, modelo Auto Regressive Conditional Heteroskedasticity (ARCH) ou autorregressivo com heteroscedasticidade condicional, por meio de filtros, que removem os componentes não lineares (transformando-a em uma série linear) e através de abordagens polinomiais.

Para séries lineares, avalia-se também o grau de estacionariedade. Se uma série é estocástica, linear e estacionária, ela pode ser modelada por técnicas estatísticas tais como AR, MA ou ARMA. No entanto, em séries não estacionárias, o modelo ARIMA pode ser utilizado.

Todas as técnicas de modelagem discutidas previamente (Seção 3.3) foram consideradas na proposta de uma metodologia para a análise de séries. Por exemplo, o modelo AR emprega uma função soma linear de observações passadas para modelagem de séries, o que permite a modelagem adequada de séries estocásticas, lineares e estacionárias.

O modelo MA considera uma função soma linear de componentes passados e aleatórios, ou também denominados ruídos, o que permite a modelagem de séries estocásticas, lineares e estacionárias. O modelo ARMA é constituído 
por AR e MA, modelando adequadamente observações e ruídos no tempo e, portanto, também é indicado para modelar séries estocásticas, lineares e estacionárias.

Ferramentas baseadas em sistemas dinâmicos e teoria do caos (Subseção 3.3.1) suportam a reconstrução de séries temporais em espaço fase (Marwan et al., 2007). Esse espaço permite a investigação dos estados de séries temporais que têm influência no tempo. Tais ferramentas buscam comportamentos determinísticos incorporados nas séries, que ao serem descobertos, simplificam a modelagem.

Séries temporais estocásticas e não lineares são, provavelmente, as mais complexas de se modelar. Elas podem ser representadas por meio de técnicas que seguem comportamentos não lineares de observações passadas, ruídos e integrações obtidas a partir da aplicação de filtros, transformando-as em séries lineares.

A partir da classificação proposta, as técnicas de modelagem (Seção 3.3) podem ser utilizadas para representação e extração do comportamento de séries temporais. Portanto, essa etapa de classificação é fundamental para a compreensão dos processos geradores de séries e, consequentemente, para a obtenção de modelos mais precisos e eficientes.

\subsection{Avaliação dos processos geradores de séries temporais}

Esta seção apresenta uma abordagem, proposta no contexto desta tese, para a avaliação dos processos geradores de séries temporais. Isso inclui a investigação de componentes relacionados à estocasticidade, linearidade e estacionariedade. Para cada componente são definidos testes de aderência que visam a classificação da série. Uma vez classificada, tal como apresentado na Figura 3.2, pode-se adotar técnicas mais adequadas para modelagem. Com objetivo de validar essa metodologia, experimentos foram conduzidos sobre um conjunto de cinco séries temporais sintéticas, descritas na Tabela 3.1.

\subsection{Avaliação de estocasticidade}

Segundo a classificação apresentada na Figura 3.2, o primeiro teste de aderência visa distinguir séries temporais determinísticas de estocásticas. Em séries determinísticas, as observações são estritamente dependentes de observações passadas, isto é, há um comportamento recorrente que pode ser desdobrado e modelado. Séries estocásticas apresentam alguma dependência com valores passados e, ainda, componentes aleatórios, que dificulta a 
Tabela 3.1: Conjunto de cinco séries temporais para estudo.

\begin{tabular}{ccc}
\hline Série Temporal & Processo Gerador & \# Observações \\
\hline \hline Caminhada Aleatória & $x_{t}=x_{t-1}+\varepsilon_{t}$ & 1.000 \\
AR(1) & $x_{t}=c+\sum_{i=1}^{p} \phi_{i} x_{t-1}+\varepsilon_{t}$ & 1.000 \\
ARIMA $(1,1,1)$ & $x_{t}=c+\sum_{i=1}^{p} \phi_{i} x_{t-i}+\phi_{p+d} x_{t-p-d}+\varepsilon_{t}+\sum_{j=1}^{q} \theta_{j} \varepsilon_{t-j}$ & 1.000 \\
mapa Logístico & $\begin{array}{c}x_{n+1}=r x_{n}\left(1-x_{n}\right) \\
r=3,8 x_{1}=0,2\end{array}$ \\
Atrator de Lorenz & dataset lor63. dat* & 1.000 \\
\hline
\end{tabular}

* http://people.virginia.edu/ smb3u/psych611/lor63.dat

modelagem.

A verificação dos níveis de estocasticidade e determinismo presentes em séries temporais pode ser obtida através de uma propriedade fundamental de sistemas dinâmicos, denominada recorrência. Essa propriedade permite caracterizar o comportamento do sistema no espaço fase, isto é, em um espaço reconstruído com maior número de dimensões ou espaço de estados (Marwan et al., 2007; Marwan, 2008). Formalmente, um sistema dinâmico é constituído por um espaço (fase), tempo contínuo ou discreto e uma regra de evolução (no tempo). Os elementos de um espaço fase representam possíveis estados do sistema. Assuma que o sistema em determinado estado em $t$ pode ser especificado por $d$ componentes (por exemplo, a quantidade de memória disponível e o comprimento da fila de processos podem representar componentes de um sistema). Esses parâmetros são, então, considerados na composição de um vetor $d$-dimensional do espaço fase, tal como definido na Equação 3.10.

$$
\vec{v}(t)=\left(v_{1}(t), v_{2}(t), \ldots, v_{d}(t)\right)^{T}
$$

A lei de evolução no tempo é uma regra que determina o estado do sistema em cada instante $t$. Para um sistema de tempo contínuo, essa regra é definida por um conjunto de equações diferenciais (Packard et al., 1980).

Em cenários experimentais, os componentes relevantes do sistema geralmente não estão totalmente disponíveis para a construção do vetor de estados. Nessa situação, a reconstrução desse sistema no espaço fase é necessária. Um método frequentemente utilizado para isso é atraso de tempo (time delay), definido na Equação 3.11, onde $m$ é a dimensão embutida e $\tau$, o atraso (ou dimensão de separação). $\overrightarrow{e_{i}}$ são vetores unitários que abrangem um sistema de coordenadas ortogonais $\left(\overrightarrow{e_{i}} \cdot \overrightarrow{e_{j}}=\delta_{i j}\right.$, onde $\delta_{i j}$ é uma função 
Kronecker $^{5}$ (Hardy e Wright, 1980) ).

$$
\vec{v}_{i}=\sum_{j=1}^{m} x_{i}+(j-1) \tau \vec{e}_{j}
$$

O teorema de imersão de Takens garante a existência de um difeomorfismo entre vetores de estado original e reconstruído (Takens, 1981). De acordo com esse teorema, todo comportamento embutido em uma série $x_{1}, x_{2}, \ldots, x_{n}$ pode ser desdobrado através de sua reconstrução em espaço multidimensional $x_{n}(m, \tau)=\left(x_{n}, x_{n+\tau}, \ldots, x_{n+m \tau}\right)$. Essa reconstrução pode ser conduzida por diferentes técnicas, nesta tese, foram consideradas as técnicas FNN e AMI para a obtenção das dimensões embutida e de separação, respectivamente. Após o cálculo dessas dimensões, é possível reconstruir o espaço fase e investigar todos os estados do sistema, bem como suas relações.

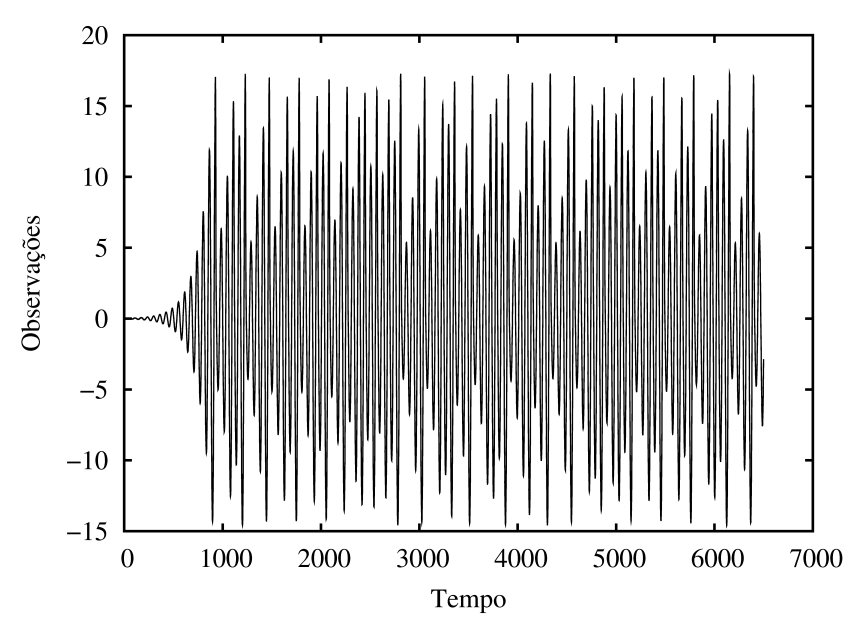

(a) Observações do sistema no tempo.

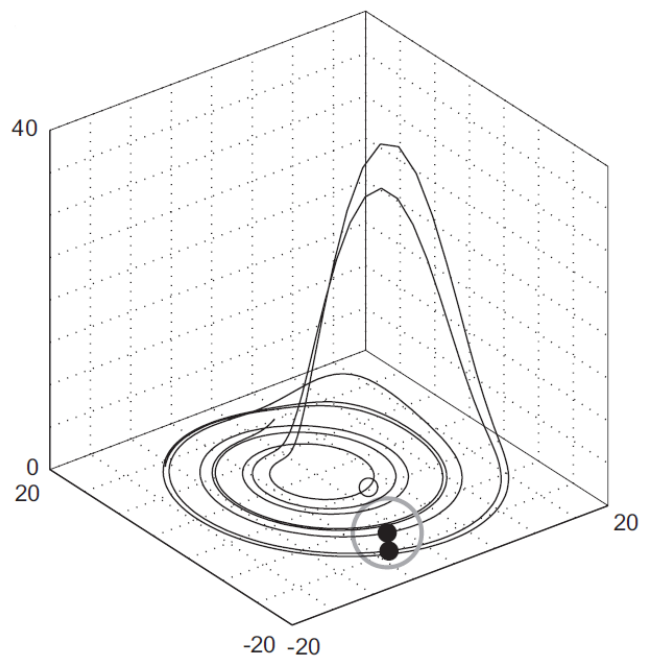

(b) Segmento de trajetória no espaço fase (Marwan et al., 2007).

Figura 3.3: Duas representações para o Sistema Rössler: a primeira ilustra como as observações estão dispostas no tempo (a) e a segunda (b), após sua reconstrução em espaço multidimensional.

A Figura 3.3(a) apresenta as observações do sistema Rössler no tempo, obtido a partir da Equação 3.12, na qual $a=0,15 ; b=0,20 ; c=10$. A avaliação visual desse sistema não permite conclusões a respeito da dificuldade de sua modelagem. Entretanto, na sua reconstrução em espaço fase (Figura 3.3(b)) é possivel identificar estados recorrentes no tempo. Após sua reconstrução, regressões da trajetória em espaço fase podem ser realizadas e, assim, determinar observações futuras com base nas anteriores.

$$
{ }^{5} \delta_{i j}=\{1|i=j ; 0| i \neq j\}
$$




$$
\begin{aligned}
& x=-y-z \\
& y=x+a y \\
& z=b+z(x-c)
\end{aligned}
$$

Além da reconstrução de séries temporais no espaço fase, é necessário verificar se essa série é determinística ou não. Uma abordagem comumente empregada na verificação do determinismo é a avaliação da propriedade de recorrência. Essa propriedade pode ser avaliada por meio do Recurrence Plot (RP) (Marwan et al., 2007). O RP avalia todos os estados de uma série que podem ser representados por uma trajetória $\left(\{\vec{x}\}_{i=1}^{N}\right.$, onde $N$ é a quantidade de estados) no espaço fase. Os valores de recorrência são armazenados em vetores, os quais são utilizados para compor uma matriz. Formalmente, essa matriz de recorrências é definida na Equação 3.13.

$$
\mathbf{R}_{i, j}=\left\{\begin{array}{l}
1: \vec{x}_{i} \approx \vec{x}_{j} \\
0: \vec{x}_{i} \not \vec{x}_{j}
\end{array} \quad i, j=1, \ldots, N\right.
$$

$\vec{x}_{i} \approx \vec{x}_{j}$ representa que um estado $x_{i}$ tende a outro $x_{j}$, segundo uma vizinhança com um limiar de distância $\varepsilon$. A matriz $\mathbf{R}_{i, j}$ compara os estados do sistema nos instantes $i$ e $j$. Se os estados são similares $\mathbf{R}_{i, j}=1$, caso contrário, os estados são diferentes $\mathbf{R}_{i, j}=0$. Um RP é definido na Equação 3.14, onde $N$ é o número de observações $\vec{x}_{i} \in \mathbb{R}^{d}$, $\varepsilon$ é um limiar de distância. $\Theta($.$) é uma$ função degrau, ou seja, $\Theta(x)=0$ se $x<0$, e $\Theta(x)=1$ caso contrário, e $\|$. \| é a norma ${ }^{6}$.

$$
\mathbf{R}_{i, j}(\varepsilon)=\Theta\left(\varepsilon-\left\|\vec{x}_{i}-\vec{x}_{j}\right\|\right), \quad i, j=1, \ldots, N
$$

Para estados $\varepsilon$-recorrentes, ou seja, estados que estão dentro de uma $\varepsilon$-vizinhança, é possível introduzir a notação definida na Equação 3.15.

$$
\vec{x}_{i} \approx \vec{x}_{j} \Longleftrightarrow \mathbf{R}_{i, j} \equiv 1
$$

O valor de $\varepsilon$ é muito importante para a determinação de recorrências no sistema. Segundo Zbilut e Webber Jr. (1992) uma estimativa razoável para $\varepsilon$ é utilizar $10 \%$ da média dos valores observados. Uma recorrência é identificada se dois segmentos de trajetória permanecem equidistantes (considerando um limiar de distância $\varepsilon$ ). A questão central é determinar o intervalo de tempo em que a trajetória permanece dentro de um $\varepsilon$-raio em torno de outra seção da trajetória (Figura 3.4). Esse intervalo depende da divergência da trajetória e do crescimento de órbitas.

\footnotetext{
${ }^{6}$ Função não negativa de valor real definida sobre um espaço vetorial, satisfazendo a desigualdade triangular.
} 


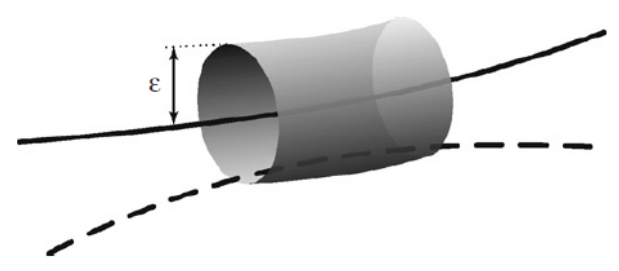

Figura 3.4: Uma diagonal no RP corresponde a uma seção da trajetória (linha tracejada) que permanece próxima à outra seção (linha sólida) ao redor de uma $\varepsilon$-vizinhança (Marwan et al., 2007).

Uma invariante ${ }^{7}$ numérica muito importante relacionada ao crescimento de órbitas é a entropia topológica $h_{D}$ (Marwan et al., 2007). Ela representa a taxa de crescimento exponencial para um certo número de órbitas distintas com uma precisão fina, porém finita. A entropia $h_{D}$ descreve a complexidade exponencial da estrutura de órbitas por meio de um único valor numérico.

A fim de definir a entropia $h_{D}$, considere $F: \mathbb{X} \rightarrow \mathbb{X}$ um mapa de espaço métrico compacto $\mathbb{X}$, com função de distância $D$. O crescimento de uma órbita $D_{n}^{F}, n=1,2,3, \ldots$, iniciando em $D_{1}^{F}=D$, é definido na Equação 3.16.

$$
D_{n}^{F}(x, y)=\max _{0 \leq i \leq n-1} D\left(F^{i}(x), F^{i}(y)\right) .
$$

$D_{n}^{F}$ mede a distância entre dois segmentos de órbita $I_{x}^{n}=\left\{x, \ldots, F^{(n-1)}(x)\right\}$ e $I_{y}^{n}=\left\{y, \ldots, F^{(n-1)}(y)\right\}$. Denota-se uma esfera ao redor de $x$ por meio de $B_{F}(x, \varepsilon, n)=\left\{y \in \mathbb{X} \mid D_{n}^{F}(x, y)<\varepsilon\right\}$.

Um conjunto $\mathbb{E} \subset \mathbb{X}$ é denominado $(n, \varepsilon)$-abrangente se $\mathbb{X} \subset \bigcup_{x \in \mathbb{E}} B_{F}(x, \varepsilon, n)$. Seja $S_{D}(F, \varepsilon, n)$ a cardinalidade mínima ou equivalente a um conjunto $(n, \varepsilon)$-abrangente. Ele representa o número mínimo de condições iniciais, cujo comportamento aproxima-se de $t$, a partir de qualquer estado inicial até $\varepsilon$.

A taxa de crescimento exponencial pode ser definida na Equação 3.17, onde $\varlimsup_{n \rightarrow \infty}$ denota o limite supremo.

$$
h_{D}(F, \varepsilon)=\varlimsup_{n \rightarrow \infty} \frac{1}{n} \log S_{D}(F, \varepsilon, n)
$$

Nota-se que $h_{D}(F, \varepsilon)$ não decai com $\varepsilon$ e a entropia topológica é definida pela Equação 3.18. Assumindo-se que $D^{\prime}$ seja uma outra métrica (ou distância) em $\mathbb{X}$, que define a mesma topologia de $D$, então, $h_{D^{\prime}}(F)=h_{D}(F)$, e a entropia topológica é uma invariante dessas entropias topológicas conjugadas (Katok e Hasselblatt, 1995).

$$
h_{D}(F)=\lim _{\varepsilon \rightarrow 0} h_{D}(F, \varepsilon) .
$$

\footnotetext{
${ }^{7}$ Uma entidade é considerada invariante sob um conjunto de transformações se a imagem transformada da entidade é indistinguivel da original. Por exemplo, a área de um triângulo é uma invariante em relação à isometria do plano Euclidiano.
} 
Isso demonstra que sistemas coordenados não apresentam variação nos valores de entropia. A partir disso, sugere-se que estruturas do RP não dependem de métricas específicas. A entropia $h_{D}$ permite caracterizar sistemas dinâmicos de acordo com sua previsibilidade, ou seja, sistemas periódicos têm $h_{D}=0$. Caso o sistema torne-se irregular, $h_{D}$ decai. Sistemas caóticos geralmente apresentam $0<h_{D}<\infty$, considerando que séries temporais originárias de processos estocásticos apresentam $h_{D}$ tendendo ao infinito.

Uma representação gráfica de $\mathrm{RP}$ é obtida por meio de sua matriz de recorrência, tal como apresentada na Figura 3.5. Um vetor de espaço fase (com pontos da trajetória) no instante $j$, que permanece dentro de uma $\varepsilon$-vizinhança (círculo cinza na Figura 3.3(b)) de outro vetor no instante $i$ é considerado um ponto de recorrência (ponto preto na trajetória da Figura 3.3(b)). Esse ponto é assinalado com um ponto preto no RP nas coordenadas $i, j$. Um vetor com valores fora da $\varepsilon$-vizinhança (círculo branco na Figura 3.3(b)) é representado por pontos brancos no RP. A Figura 3.5 apresenta o RP do sistema Rössler.

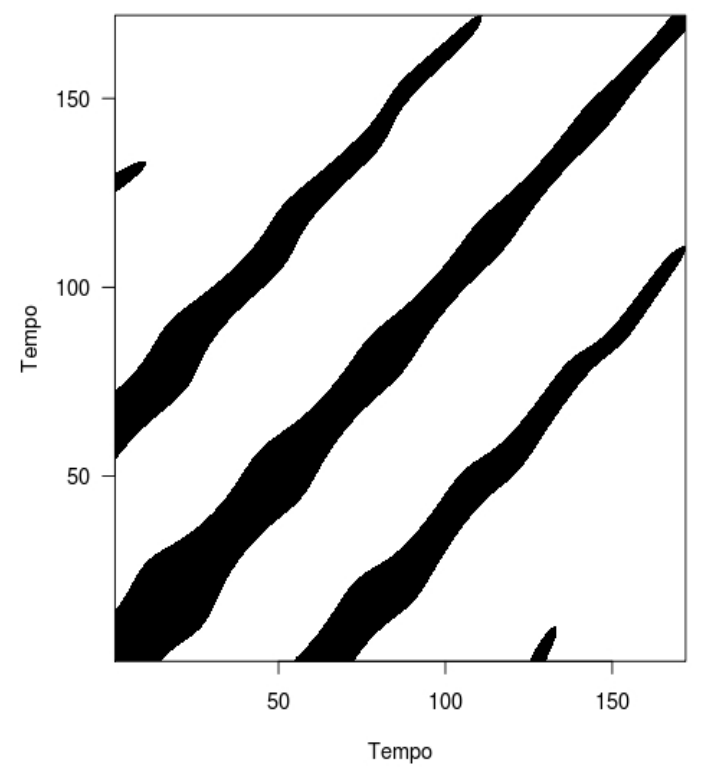

Figura 3.5: Uma representação gráfica de Recurrence Plot do sistema Rössler obtida a partir de sua matriz de recorrências.

Ambos os eixos do RP representam o tempo, caso $\left.\mathbf{R}_{i, i} \equiv 1\right|_{i=1} ^{N}$, o RP tem sempre uma linha diagonal principal denominada line of identity (LOI). Além disso, o RP é simétrico, por definição, caso apresente diagonais principais onde $\mathbf{R}_{i, j} \equiv \mathbf{R}_{j, i}$.

Ao analisar um RP, nota-se a presença de estruturas bem definidas, denominadas texturas, que podem ser classificadas em: pontos recorrentes isolados, linhas diagonais, verticais e horizontais. Os pontos isolados podem ocorrer quando os estados são raros ou persistem por um período muito curto de tempo. Um exemplo de pontos isolados é apresentado na Figura 3.8. 
As linhas diagonais ocorrem quando um segmento de trajetória permanece paralelo a outro, ou seja, localizam-se na mesma $\varepsilon$-vizinhança (Figura 3.4), por $l$ instantes de tempo. O comprimento das diagonais determina o intervalo de tempo em que segmentos da trajetória evoluem, mantendo-se equidistantes. As linhas verticais (horizontais) correspondem a um intervalo de tempo no qual o estado não se altera ou se modifica muito pouco.

Com base em texturas obtidas, Zbilut e Webber Jr. (1992) quantificam a estrutura do RP, definindo uma metodologia denominada Recurrence Quantification Analysis (RQA), que estabelece as seguintes medidas: Taxa ou percentual de recorrência (RR), percentual de determinismo (DET), comprimento máximo de linha ( $L_{\max }$ ), divergência (DIV), Entropia de Shannon da distribuição do comprimento de linha (ENTR).

Essas medidas são baseadas na densidade dos estados recorrentes, nas estruturas de linhas verticais e diagonais do RP. O cálculo dessas medidas, por meio de matrizes da trajetória ao longo do LOI, produz um comportamento dependente do tempo. Um dos objetivos dessas medidas é identificar pontos de bifurcação, especialmente, transições (Marwan et al., 2007).

A primeira medida RQA é a taxa ou percentual de recorrência, que mede a densidade dos estados recorrentes, definida na Equação 3.19, onde $N$ representa o número de observações.

$$
\operatorname{RR}(\varepsilon)=\frac{1}{N^{2}} \sum_{i, j=1}^{N} \mathbf{R}_{i, j}(\varepsilon)
$$

Na medida que $N \rightarrow \infty$, RR representa a probabilidade de um estado $x_{i}$ recorrer em sua $\varepsilon$-vizinhança no espaço fase. A Tabela 3.2 apresenta os valores de RR para as cinco séries temporais definidas previamente na Seção 3.2. Nota-se que, incrementando-se $\varepsilon$, o valor de RR também aumenta, isso porque o espaço Euclidiano torna-se mais abrangente e a probabilidade de estados recorrentes é maior.

Tabela 3.2: Valores de RR para cinco séries temporais.

\begin{tabular}{ccccc}
\hline Série & $\varepsilon=0,1$ & $\varepsilon=0,5$ & $\varepsilon=1,0$ & $\varepsilon=\frac{\mu}{10}$ \\
\hline \hline Caminhada Aleatória & 0,0 & 0,000184078 & 0,00115211 & 0,0121925 \\
AR(1) & 0,0 & 0,000233572 & 0,00618351 & 0,0 \\
ARIMA(1,1,1) & 0,0 & $2,20676 e-06$ & 0,07929 & $1,32406 e-05$ \\
Mapa Logístico & 0,0003026580 & 0,136895 & 0,857076 & $5,11848 e-05$ \\
Atrator de Lorenz & $1,15766 e-05$ & 0,00032013 & 0,00135383 & $2,04705 e-06$ \\
\hline
\end{tabular}

Medidas baseadas em estruturas de diagonais permitem quantificar o grau de determinismo (ou previsibilidade) de séries. Processos cujo comportamento é não correlacionado, fracamente correlacionado ou estocástico apresentam nenhum ou número reduzido de linhas diagonais. Por outro lado, processos 
determinísticos apresentam diagonais longas e formação de texturas, bem como pontos isolados de recorrência. Dessa maneira, a taxa de estados recorrentes que formam a estrutura de diagonais é definida na Equação 3.20, onde $P(\varepsilon, l)$ é o histograma obtido a partir do comprimento $l$ de linhas diagonais, definida na Equação 3.21.

$$
\begin{gathered}
\mathrm{DET}=\frac{\sum_{l=l_{\min }}^{N} l P(\varepsilon, l)}{\sum_{l=1}^{N} l P(\varepsilon, l)} \\
P(\varepsilon, l)=\sum_{i, j=1}^{N}\left(1-\mathbf{R}_{i-1, j-1} \varepsilon\right)\left(1-\mathbf{R}_{i+l, j+l} \varepsilon\right) \prod_{k=0}^{l-1} \mathbf{R}_{i+k, j+k} \varepsilon
\end{gathered}
$$

A medida DET representa o determinismo (ou previsibilidade) do sistema. O limite $l_{\min }$ exclui as linhas diagonais de comprimento inferior a $l_{\min }$. Para $l_{\min }=1$ o determinismo é 1 . A escolha de $l_{\min }$ deve considerar o histograma $P(\varepsilon, l)$, que pode tornar-se esparso, caso $l_{\min }$ seja muito grande, e, assim, a representatividade de DET decai.

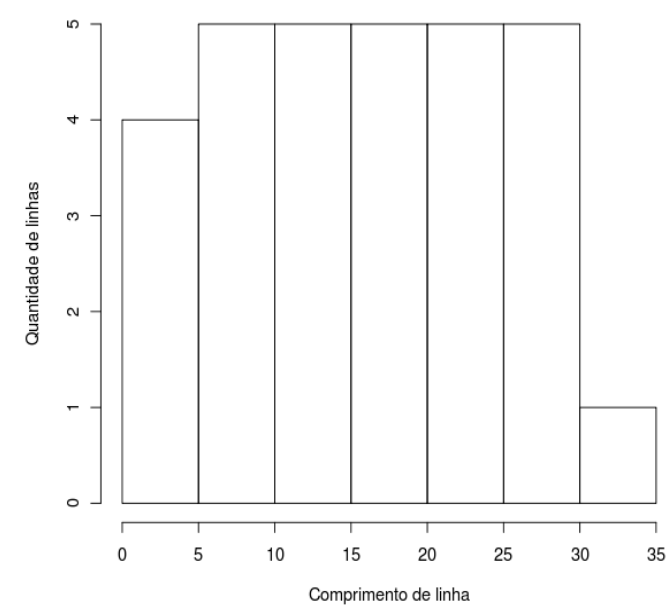

Figura 3.6: Um exemplo de histograma das diagonais de um RP.

A Figura 3.6 apresenta como um histograma $P(\varepsilon, l)$ é construído, considerando o comprimento das diagonais do RP. A Tabela 3.3 apresenta os valores de DET para as cinco séries temporais definidas previamente.

Uma diagonal de comprimento $l$ significa que um segmento de trajetória é muito próximo durante $l$ instantes de tempo de outro segmento. Dessa maneira, tais linhas estão relacionadas com a divergência da trajetória. O comprimento médio de diagonais é definido na Equação 3.22. 
Tabela 3.3: Valores de DET para cinco séries temporais com $L_{\min }=2$.

\begin{tabular}{ccccc}
\hline Série & $\varepsilon=0,1$ & $\varepsilon=0,5$ & $\varepsilon=1,0$ & $\varepsilon=\frac{\mu}{10}$ \\
\hline \hline Caminhada Aleatória & 0,0 & 0,000184078 & 0,00115211 & 0,0121925 \\
AR(1) & 0,0 & 0,000233572 & 0,00618351 & 0,0 \\
ARIMA(1,1,1) & 0,0 & 0,0 & 0,0434783 & 0,0 \\
Mapa Logístico & 0,242647 & 0,475063 & 0,991169 & 0,0869565 \\
Atrator de Lorenz & 0,951578 & 0,948876 & 0,95001 & 0,963325 \\
\hline
\end{tabular}

$$
L=\frac{\sum_{l=l_{\min }}^{N} l P(\varepsilon, l)}{\sum_{l=l_{\min }}^{N} P(\varepsilon, l)}
$$

Esse comprimento representa o período em que duas trajetórias permanecem próximas uma da outra, e pode ser interpretado como o tempo médio previsível. Outra medida RQA considera o maior comprimento $L_{\max }$ das diagonais definida na Equação 3.23. Essa medida representa o maior período de tempo em que duas trajetórias permanecem $\varepsilon$ equidistantes, onde, $N_{l}=\sum_{l>l_{\min }} P(\varepsilon, l)$ é o comprimento total das diagonais.

$$
L_{\max }=\max \left(\left\{l_{i}\right\}_{i=1}^{N_{l}}\right)
$$

A próxima medida refere-se ao grau de divergência obtido aplicando-se o inverso da medida $L_{\max }$, definido na Equação 3.24.

$$
\mathrm{DIV}=\frac{1}{L_{\max }}
$$

Essas medidas estão relacionadas com a divergência do espaço fase da trajetória. Quanto mais rápido os segmentos de trajetória divergem, menores são as linhas diagonais e maior é o valor de DIV. A Tabela 3.4 apresenta os valores de DIV para as cinco séries temporais definidas previamente (Tabela 3.1).

Tabela 3.4: Valores de DIV para as cinco séries temporais em estudo.

\begin{tabular}{ccccc}
\hline Série & $\varepsilon=0,1$ & $\varepsilon=0,5$ & $\varepsilon=1,0$ & $\varepsilon=\frac{\mu}{10}$ \\
\hline \hline Caminhada Aleatória & 0,0 & 0,5 & 0,25 & 0,0102041 \\
AR(1) & 0,0 & 1,0 & 0,5 & 0,0 \\
ARIMA(1,1,1) & 0,0 & 1,0 & 0,5 & 1,0 \\
Mapa Logístico & 0,333333 & 0,1 & 0,00740741 & 0,5 \\
Atrator de Lorenz & 0,007194 & 0,003876 & 0,003759 & 0,010101 \\
\hline
\end{tabular}

Além dessas medidas, uma abordagem eficiente para medir a quantidade de informação e, consequentemente, a complexidade embutida em séries 
temporais, é a Entropia de Shannon definida na Equação 3.25, onde $p(l)=\frac{P(l)}{N_{l}}$.

$$
\mathrm{ENTR}=-\sum_{l=l_{\min }}^{N} p(l) \log p(l) .
$$

A Entropia reflete a complexidade do RP em termos da formação das linhas diagonais, ou seja, para estados não correlacionados o valor de ENTR é baixo, indicando menor complexidade. Nota-se que essa medida permite analisar o quanto cada comprimento de linha contribui no total $L_{\max }$. Quanto maior o valor de ENTR, mais segmentos de tamanho distintos compõem a textura do RP. A Tabela 3.5 apresenta os valores de ENTR para as cinco séries temporais previamente definidas.

Tabela 3.5: Valores de ENTR para as cinco séries temporais em estudo.

\begin{tabular}{ccccc}
\hline Série & $\varepsilon=0,1$ & $\varepsilon=0,5$ & $\varepsilon=1,0$ & $\varepsilon=\frac{\mu}{10}$ \\
\hline \hline Caminhada Aleatória & 0,0 & 0,0 & 0,585966 & 1,68495 \\
AR(1) & 0,0 & 0,0 & 0,0 & 0,0 \\
ARIMA(1,1,1) & 0,0 & 0,0 & 0,0 & 0,0 \\
Mapa Logístico & 1,673 & 1,955 & 2,891 & 0,0 \\
Atrator de Lorenz & 2,538 & 2,481 & 2,498 & 1,77118 \\
\hline
\end{tabular}

Todas as medidas RQA descritas previamente contribuem para análise de estocasticidade em séries temporais. Por exemplo, nas séries Caminhada Aleatória e $\operatorname{ARIMA}(1,1,1)$, os respectivos valores de RR demonstram baixo percentual de recorrência, o que de fato é esperado, assumindo-se que tais séries são oriundas de processos geradores estocásticos (ver Tabela 3.1). Entretanto, o RR para o atrator de Lorenz é baixo, o que não condiciona a classificá-lo como estocástico, para isso outras medidas RQA devem ser analisadas.

Essa relação entre estocasticidade e o RR pode ser confirmada na análise de outras medidas para a série Caminhada Aleatória, sumarizadas na Tabela 3.6. Assim, ao avaliar os valores da Tabela 3.6, nota-se que essa série tem baixo grau de determinismo (DET), alto grau de divergência (DIV) e baixa complexidade (ENTR).

Além disso, ao analisar o gráfico RP dessa série, apresentado na Figura 3.7, confirma-se a baixa frequência de diagonais. Dadas essas avaliações, a série temporal Caminhada Aleatória pode ser classificada como estocástica. 
Tabela 3.6: Valores das medidas RQA para série Caminhada Aleatória com dimensão de separação 1 , embutida 3 e distância $\varepsilon=$ 1,0 .
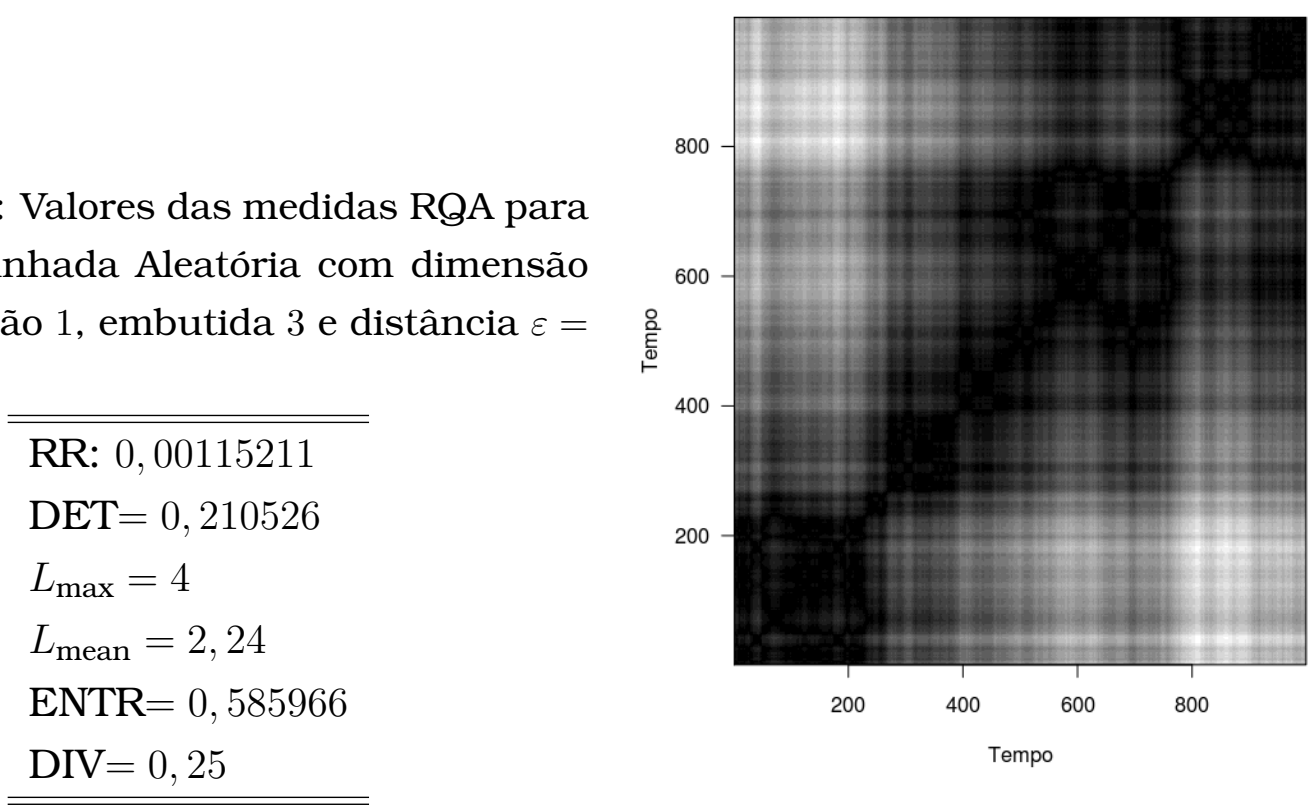

Figura 3.7: Gráfico RP da série Caminhada Aleatória.

Dada a série temporal AR(1), descrita na Seção 3.2, e analisando os resultados das quatro medidas RQA, sumarizadas na Tabela 3.7, pode-se confirmar que AR(1) tem baixo percentual de estados recorrentes (RR). Portanto, essa série tem baixo grau de determinismo (DET), elevado grau de divergência (DIV) e baixa complexidade ENTR.

Além disso, ao analisar o gráfico RP da série, apresentado na Figura 3.8, confirma-se a baixa frequência de diagonais. Dados os resultados das métricas RQA (Tabela 3.7) e do gráfico RP (Figura 3.8), AR(1) pode ser classificada como uma série estocástica.

Tabela 3.7: Valores das medidas RQA para série AR(1) com dimensão de separação 3, embutida 5 e distância $\varepsilon=1,0$.
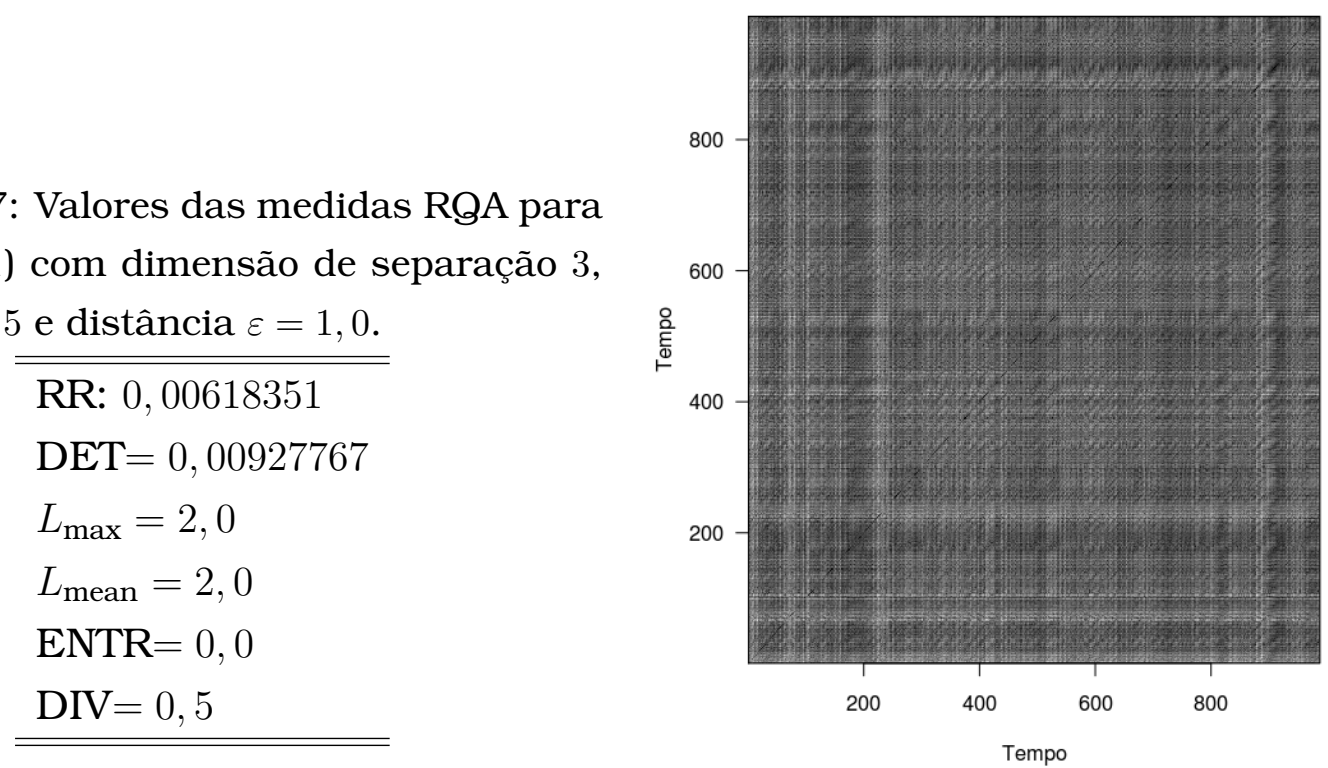

Figura 3.8: Gráfico RP da série AR(1).

Considere a série temporal $\operatorname{ARIMA}(1,1,1)$, descrita na Seção 3.2, e seus resultados para as medidas RQA, sumarizadas na Tabela 3.8. Pode-se 
observar que essa série tem baixo percentual de estados recorrentes (RR), portanto, ela apresenta baixo grau de determinismo (DET), elevado grau de divergência (DIV) e complexidade ENTR nula. Acredita-se que esse valor de ENTR está relacionado com a integração dos modelos AR e MA na composição de $\operatorname{ARIMA}(1,1,1)$.

Além disso, ao analisar o gráfico RP dessa série, apresentado na Figura 3.9, confirma-se a baixa frequência de diagonais e formação de textura. Entretanto, o formato da textura não apresenta diagonais longas, mas sim regiões do gráfico com maior concentração de estados recorrentes. Dados os resultados das métricas RQA e do gráfico RP, $\operatorname{ARIMA}(1,1,1)$ pode ser classificada como uma série estocástica.

Tabela 3.8: Valores das medidas RQA para série $\operatorname{ARIMA}(1,1,1)$ com dimensão de separação 12 , embutida 5 e distância $\varepsilon=$ $1,0$.

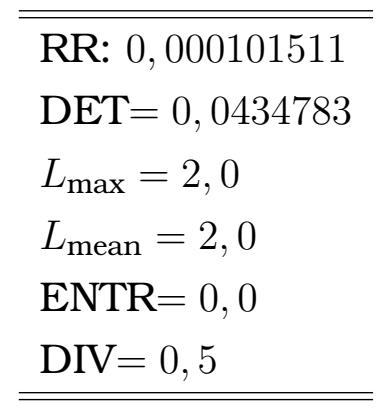

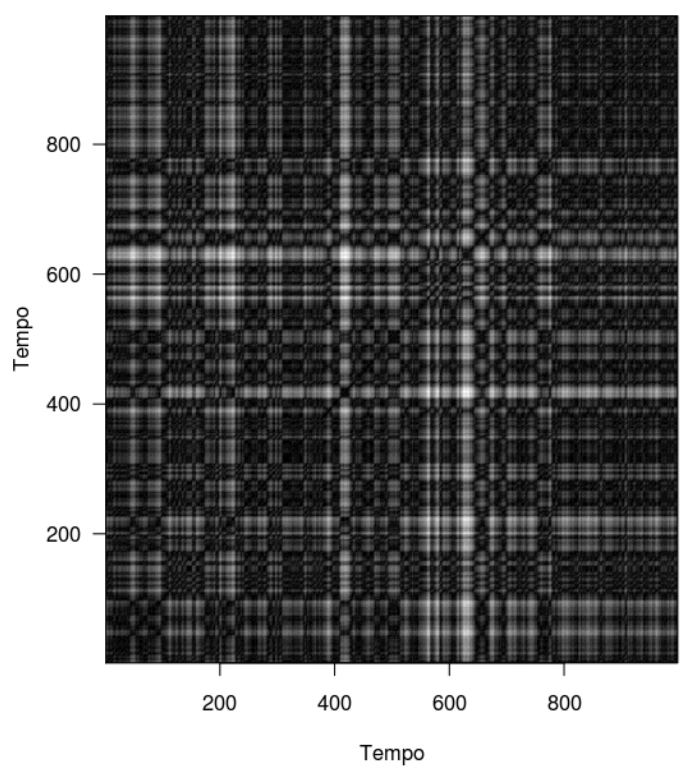

Figura 3.9: Gráfico RP da série $\operatorname{ARIMA}(1,1,1)$.

Considere uma série temporal cujas observações foram extraídas de um mapa Logístico, descrita na Tabela 3.1, e analisando os resultados das quatro medidas RQA, sumarizadas na Tabela 3.9, pode-se confirmar que essa série tem grande percentual de estados recorrentes (RR). Portanto, essa série tem alto grau de determinismo (DET), baixo grau de divergência (DIV) e complexidade (ENTR) elevada.

Além disso, ao analisar seu gráfico RP, apresentado na Figura 3.10, confirma-se a baixa frequência de diagonais, no entanto há formação de uma textura bem definida. O formato dessa textura apresenta uma estrutura uniforme, o que caracteriza um comportamento determinístico. Dados os resultados das métricas RQA e do gráfico RP, as observações extraídas do mapa Logístico podem ser classificadas como uma série temporal determinística. 
Tabela 3.9: Valores das medidas RQA para série mapa Logístico com dimensão de separação 3 , embutida 5 e distância $\varepsilon=1,0$.

$$
\begin{aligned}
& \hline \hline \text { RR: } 0,857076 \\
& \text { DET }=0,991169 \\
& L_{\text {max }}=135 \\
& L_{\text {mean }}=7,9072 \\
& \text { ENTR }=1,388 \\
& \text { DIV }=2.65431
\end{aligned}
$$

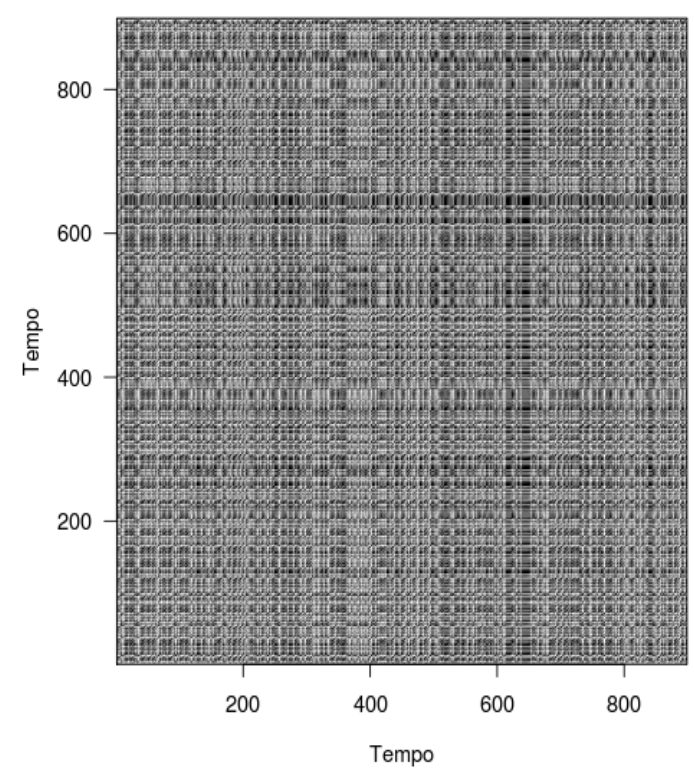

Figura 3.10: Gráfico RP da série mapa Logístico.

Considere uma série temporal cujas observações foram extraídas do atrator de Lorenz, descrito na Tabela 3.1. Analisando os resultados das quatro medidas RQA, sumarizadas na Tabela 3.10, pode-se confirmar que essa série tem grande percentual de estados recorrentes (RR). Portanto, o atrator de Lorenz é caracterizado por alto grau de determinismo (DET), baixo grau de divergência (DIV) e complexidade (ENTR) elevada.

Além disso, ao analisar o gráfico RP, apresentado na Figura 3.11, confirma-se a grande frequência de linhas diagonais e a formação de uma textura bem definida. Dados os resultados das métricas RQA e do gráfico $\mathrm{RP}$, a série formada pelas observações extraídas do atrator de Lorenz pode ser classificada como determinística. 
Tabela 3.10: Valores das medidas RQA para série atrator de Lorenz com dimensão de separação 5 , embutida 3 e distância $\varepsilon=1,0$.
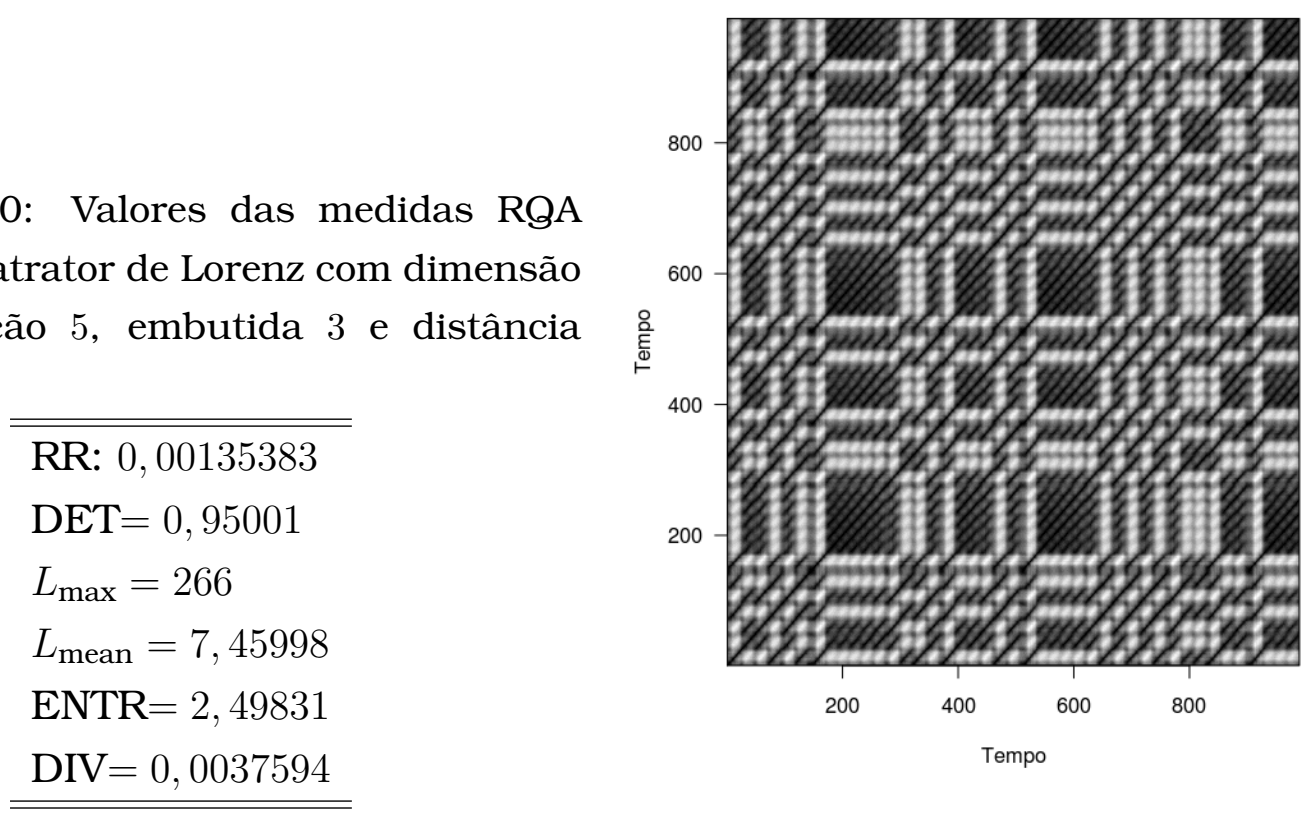

Figura 3.11: Gráfico RP da série atrator de Lorenz.

Os resultados apresentados pela abordagem RP proveem mecanismos que favorecem a escolha de modelos mais adequados para a análise de comportamento de séries temporais. Pode-se inferir, por exemplo, que séries com alto grau de determinismo são modeladas adequadamente utilizando-se ferramentas de sistemas dinâmicos. Tomando-se, ainda, como exemplo as séries Lorenz e AR(1), os resultados da métrica DET são 0,95001 e 0,00927767, respectivamente. A partir desses resultados pode-se optar por técnicas determinísticas, disponíveis pela área de sistemas dinâmicos e teoria do caos, para análise da série Lorenz e por ferramentas estatísticas para a análise da série $\operatorname{AR}(1)$.

\subsubsection{Avaliação de linearidade}

A determinação da componente aleatória em séries temporais, conforme apresentado na Subseção 3.3.2, auxilia na escolha do modelo, determinístico ou estocástico, a ser adotado. No entanto, além da determinação dessa componente, séries temporais podem ser classificadas de acordo com seu processo gerador em: linear ou não linear.

Em sistemas que apresentam comportamento linear, o valor de uma observação está diretamente relacionado, por meio de uma função de transferência $v(t)$, com valores passados (Morettin e Toloi, 2006). Formalmente, uma observação $x_{t}$ pode ser determinada por um filtro linear definido por $x_{t}=\sum_{k=0}^{\infty} v(t) x_{t-k}$, sendo $k$ o número de observações passadas consideradas. Nesse tipo de série, um dos desafios da modelagem é estimar a função de transferência $v(t)$, que permite predizer valores futuros e estudar o 
comportamento do sistema (Morettin e Toloi, 2006).

Em diversas situações, principalmente, na análise de sistemas reais, séries temporais podem ter comportamento diferente de um processo linear. Observações dessas séries, denominadas não lineares, tem comportamento variável no decorrer da coleta, tal como mudanças repentinas, irreversibilidade ou, ainda, variância condicional. Séries não lineares são geralmente representadas por modelos específicos ou por meio da aplicação de filtros, que suavizam e/ou removem a componente não linear, transformando a série em um processo linear (Morettin e Toloi, 2006). No entanto, uma das grandes dificuldades no processo de modelagem de séries temporais é determinar se o processo gerador da série é ou não definido por uma função linear.

Nesse sentido, Lee et al. (1993) propuseram um teste de linearidade, baseado em White (1989, 1990), denominado White Neural Network (WNN), para séries temporais que emprega conceitos de redes neurais. Dado um processo estocástico $Z=\left\{Z_{t}, t=1, \ldots, n\right\}, Z_{t}=\left(Y_{t}, X_{t}\right)$, onde $Y_{t}$ é um escalar e $X_{t}$ é um vetor, investiga-se o relacionamento entre esses dois componentes em termos de probabilidade condicional, ou seja, a probabilidade de ocorrer $Y_{t}$ dado $X_{t}$, denotado $E\left(Y_{t} \mid X_{t}\right)$. Formalmente, isso pode ser representado por uma função regressiva $g(x)=E\left(Y_{t} \mid X_{t}\right)$. O objetivo do teste é verificar a adequabilidade da arquitetura da rede neural WNN para a representação do mapeamento da função $g($.$) .$

WNN emprega um modelo de rede neural supervisionada (feedforward) com uma única camada intermediária (ou escondida), tal como apresentada na Figura 3.12.

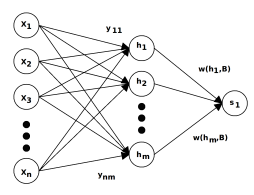

Figura 3.12: Um exemplo de rede neural supervisionada com uma única camada escondida.

Nessa rede, as unidades de entrada $x_{i} i=1, \ldots, n$ enviam sinais através das conexões, que atenuam ou amplificam o sinal original por um fator $\gamma_{j i}$ (pesos). Uma unidade de processamento intermediária (ou neurônio) $j$ soma os sinais recebidos da camada de entrada no formato $\hat{x}^{\prime} \gamma_{j}$, onde $\hat{x}\left(1, x_{1}, \ldots, x_{n}\right)^{\prime}, \gamma_{j} \approx$ $\left(\gamma_{j 0}, \gamma_{j 1}, \ldots, \gamma_{j k},\right)^{\prime}$, e produz uma saída $\psi\left(\hat{x}^{\prime} \gamma_{j}\right)$, onde a função de ativação $\psi$ é um mapeamento não linear de $\mathbb{R}$ em $\mathbb{R}$. Geralmente, $\psi$ é uma função de distribuição cumulativa (c.d.f), neste trabalho foi adotada uma logística não linear, $\psi(\lambda)=\left(1+e^{-\lambda}\right)^{-1}, \lambda \in \mathbb{R}$. A camada de saída faz um somatório dos sinais recebidos da camada intermediária, ou seja, $\psi\left(\hat{x}^{\prime} \gamma_{j}\right), j=1, \ldots, m$, 
representada na Equação 3.26, onde, $\theta \equiv \theta\left(\beta^{\prime}, \gamma^{\prime}\right)^{\prime} . \beta=\left(\beta_{0}, \ldots, \beta_{m}\right)^{\prime}$ são pesos das conexões entre as camadas intermediária e de saída e $\gamma=\left(\gamma_{1}, \ldots, \gamma_{m}\right)^{\prime}$ correspondem aos pesos iniciais fornecidos à rede.

$$
f(x, \theta)=\beta_{0}+\sum_{j=1}^{m} \beta_{j} \psi\left(\hat{x}^{\prime} \gamma_{j}\right) \quad m \in \mathbb{N}
$$

Para um dado conjunto $\theta$ de pesos, tem-se $Y_{t}=f\left(X_{t}, \theta\right)+\left[g\left(X_{t}-f\left(X_{t}, \theta\right)\right]+\left[Y_{t}-\right.\right.$ $\left.g\left(X_{t}\right)\right]=f\left(X_{t}, \theta\right)+a\left(X_{t}, \theta\right)+\epsilon_{t}=f\left(X_{t}, \theta\right)+\mu\left(X_{t}, \theta\right)$, onde $a\left(X_{t}, \theta\right) \equiv g\left(X_{t}\right)-f\left(X_{t}, \theta\right)$ é o erro aproximado da saída da rede para $g\left(X_{t}\right)$. O termo $\epsilon_{t} \equiv Y_{t}-g\left(X_{t}\right)$ consiste no erro aleatório, no qual $E\left(\epsilon_{t} \mid X_{t}\right)=0$ e, por fim, $\mu\left(X_{t}, \theta\right) \equiv a\left(X_{t}, \theta\right)+\epsilon_{t}$ é o componente residual, que consiste dos dois erros: aproximado e aleatório.

A rede WNN considera uma função de ativação não linear. Portanto, essa rede é capaz de representar uma função $g($.$) , ou seja, o erro aproximado$ é pouco representativo. Neste caso, existe um vetor de pesos $\theta^{*}$ tal que $g\left(X_{t}\right)=f\left(X_{t}, \theta^{*}\right)$, com probabilidade 1. Isso significa que, após o treinamento da rede, erros de aproximação tendem a ser reduzidos ou removidos por completo. Entretanto, erros aleatórios (outro componente residual) podem estar presentes, indicando que as entradas fornecidas à rede tem um certo grau de comportamento linear associado.

Formalmente, podem-se definir testes de hipóteses a fim de verificar a linearidade de séries temporais. A hipótese nula é representada na Equação 3.27. A hipótese alternativa define que a rede WNN não representa $g($.$) e pode ser expressa na Equação 3.28.$

$$
\begin{gathered}
H_{0}: P\left[g\left(X_{t}\right)=f\left(X_{t}, \theta\right)\right]=1 \text { para algum } \theta^{*} \\
H_{a}: P\left[g\left(X_{t}\right)=f\left(X_{t}, \theta\right)\right]<1 \text { para todo } \theta^{*}
\end{gathered}
$$

Alguns estudos Stinchcombe e White (1989); White (1989, 1990) demonstram que funções não lineares similares a $\psi$ (Equação 3.26) fornecem aproximações precisas para a determinação de não linearidade. Além disso, na medida que $m$ tende a zero determinadas funções podem, erroneamente, serem classificadas como lineares ao invés de não lineares e vice-versa. Gallant e White (1992) adotam em seu estudo sobre o modelo Mackey-Glass cinco neurônios na camada intermediária. Neste trabalho, adotou-se $m=10$ nos testes de aderência Hegger et al. (1998).

A WNN tem bons resultados se $\sum_{j=1}^{m} \beta_{j} \psi\left(\hat{x}^{\prime} \gamma_{j}\right)$ são capazes de extrair valores com erro Chi-Quadrado $e_{t}^{*}=Y_{t}-\hat{X}_{t}^{\prime} \theta^{*}$, onde $\theta^{*}$ é um vetor de parâmetros de aproximação linear quadrática para $E\left(Y_{t} \mid X_{t}\right)$, dado por $\theta^{*}=\left(\beta_{i}^{*}, \gamma_{j}^{*}\right)$. Stinchcombe e White (1989) demostram que ao utilizar $\psi$ como uma c.d.f. 
logística, o termo $\psi\left(\hat{x}^{\prime} \gamma_{j}\right)$ é capaz de extrair valores de erro que, posteriormente, podem ser aplicados em testes de hipóteses.

Sabendo que a rede neural WNN aproxima funções não lineares, ela pode ser empregada na verificação de linearidade em séries temporais. A fim de demonstrar o funcionamento do teste WNN, considere as cinco séries temporais apresentadas na Tabela 3.1. Para cada uma dessas séries, emprega-se o teste Chi-Quadrado que verifica se a saída da WNN satisfaz a hipótese alternativa, $H_{a}$, ou seja, a série é linear. Neste caso, o nível de significância é $\alpha=0,05$ ou $5 \%$. Dessa maneira, tem-se as seguintes hipóteses:

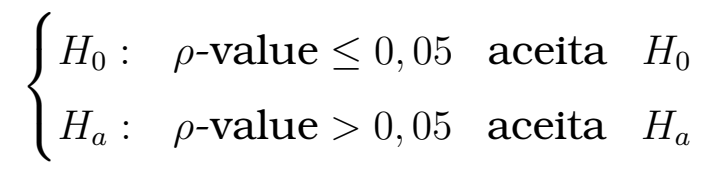

A Tabela 3.11 sumariza os resultados do teste WNN para as séries temporais $\operatorname{AR}(1), \operatorname{ARIMA}(1,1,1)$, Caminha Aleatória, Mapa Logístico e Atrator de Lorenz. Cada linha dessa tabela corresponde aos resultados de $\rho$-value, hipótese nula e a classificação (linear ou não linear).

Tabela 3.11: Resultados do teste WNN para identificação linearidade em cinco séries temporais.

\begin{tabular}{c|ccc}
\hline Série & $\rho$-value(WNN) & Hipótese nula $H_{0}$ & Linearidade \\
\hline \hline AR(1) & 0,8 & Rejeita & Linear \\
$\begin{array}{c}\text { ARIMA(1,1,1) } \\
\text { Caminhada }\end{array}$ & 0,3 & Rejeita & Linear \\
$\begin{array}{c}\text { Aleatória } \\
\text { Mapa }\end{array}$ & 0,5 & Rejeita & Linear \\
$\begin{array}{c}\text { Logístico } \\
\text { Atrator } \\
\text { Lorenz }\end{array}$ & $<0,0001$ & Aceita & Não linear \\
\hline
\end{tabular}

\subsubsection{Avaliação de estacionariedade}

Após a aplicação da abordagem RP, uma série temporal pode ser classificada em determinística ou estocástica. Para séries determinísticas, a utilização de ferramentas disponíveis pela área de sistemas dinâmicos e teoria do caos fornecem resultados satisfatórios. Entretanto, para séries classificadas como estocásticas, apenas essa informação não garante uma modelagem eficiente.

Dessa maneira, o teste WNN (apresentado na Subseção 3.3.2) visa identificar a presença ou não de componentes lineares em séries temporais estocásticas, o que auxilia na modelagem. Além disso, outro fator importante para análise de séries temporais é a determinação de sua estacionariedade. 
Séries estacionárias são caracterizadas por equilíbrio estatístico em torno de uma média $\mu$ e uma dispersão constante $\sigma^{2}$. Existem duas categorias de séries estacionárias: fracamente estacionária ou de segunda ordem e estritamente estacionária. Uma série estacionária de segunda ordem tem média e variância constantes. Séries estritamente estacionárias apresentam, além de média e variância constantes, uma estrutura de autocovariância também constante. Se uma série apresenta estacionariedade de covariância, tal comportamento se repete ao longo do tempo, ou seja, a autocovariância permanece a mesma, independentemente do ponto de referência temporal.

Dadas essas circunstâncias, a autocovariância depende apenas da diferença entre dois estados de referência temporal, ou seja $\left|x_{t_{2}}-x_{t_{1}}\right|$ e não dos valores absolutos $x_{1}$ e $x_{2}$. Portanto, se uma série é estacionária, a magnitude de autocorrelação atenua rapidamente, entretanto, se uma série é não estacionária, a autocorrelação diminui gradualmente ao longo do tempo.

Considere uma série temporal discreta com valores observados $x_{t_{1}}, x_{t_{2}}, \ldots, x_{t_{n}}$, e os pares de pontos $\left(x_{1}, x_{k+1}\right),\left(x_{2}, x_{k+2}\right), \ldots,\left(x_{k-n}, x_{n}\right)$, que podem ser representadas por $(n-k)$ observações de uma f.d.p. (função de densidade de probabilidade) definida por $f_{12}\left(x_{1}, x_{2}\right)$ conjunta, similar em todos os $k \Delta$ $(k \in[1, n-k])$ instantes futuros. De maneira geral, uma condição de equilíbrio implica que as propriedades multivariáveis das f.d.p's associadas com qualquer conjunto $x_{t_{1}}, x_{t_{2}}, \ldots, x_{t_{n}}$ dependem somente das diferenças $\left|t_{i}-t_{j}\right|$, definida na Equação 3.29. Para todos os conjuntos de séries e para todos os desdobramentos $k$, um processo estocástico que satisfaça as condições da Equação 3.29 é considerado estritamente estacionário.

$$
F_{X_{t_{1}} \ldots X_{t_{n}}}\left(x_{t_{1}}, x_{t_{2}}, \ldots, x_{t_{n}}\right)=F_{X_{t_{1+k}} \ldots X_{t_{n+k}}}\left(x_{t_{1}}, x_{t_{2}}, \ldots, x_{t_{n}}\right)
$$

Duas estratégias diferentes podem ser aplicadas na verificação de estacionariedade: considerando ferramentas de sistemas dinâmicos ou estatísticas. A área de sistemas dinâmicos busca identificar estacionariedade por meio de técnicas que avaliam trajetórias e complexidade de informações, tais como o expoente de Lyapunov, correlações, e entropias (Kolmogorov, etc.). Ferramentas estatísticas visam avaliar se a probabilidade estrutural de uma série é ou não afetada após deslocamentos no tempo.

Uma ferramenta de sistemas dinâmicos comumente aplicada na avaliação de estacionariedade é o Space-Time-Separation Plot (STP). O STP é um método de correlação que visa identificar se uma série tem trajetória geométrica (denominada global) ou estrutura de fractais (denominada local). Para isso, empregam-se estimativas associadas à dimensão de correlação.

Do ponto de vista estatístico, pode-se considerar uma função de autocorrelação (ACF) que mede o grau de correlação de uma série temporal, 
ou seja, calcula a distância de separação de uma observação no instante $t$ com uma outra no instante $t+k$, onde $k$ é um deslocamento. Isso permite analisar o grau de irregularidade do conjunto de dados. Formalmente, considere uma série $X$, com média $\mu$, a autocorrelação $\operatorname{ACF}(k)$ é definida pela Equação 3.30, onde, $E[]$ é o valor esperado médio da expressão e $\sigma^{2}$ é a variância de $X$.

$$
\operatorname{ACF}(k)=\frac{E\left[\left(X_{t}-\mu\right)\left(X_{t+k}-\mu\right)\right]}{\sigma^{2}}
$$

O STP considera a separação de tempo dos estados de forma explícita, ou seja, por meio da dispersão (distância) entre dois estados no espaço versus sua separação no tempo. Ele gera contornos que determinam os intervalos de correlação para uma dada dimensão embutida de uma série. Essa correlação representa a probabilidade de ocorrência de um par de estados, aleatoriamente escolhido, ser menor que uma distância $r$ no espaço fase. Essa distância e a dimensão de separação refletem o posicionamento na vizinhança em um dado instante de tempo.

Em particular, todos os pares de estados separados no tempo $\Delta t$ estão separados no espaço fase por uma distância $\operatorname{ACF}_{p}(\Delta t)$ com probabilidade $p$. Dessa maneira, os contornos mapeiam uma fração de pontos mais próximos (em termos da distância $r$ ) para uma dada separação $\Delta t$, equivalente a $P(\mid x(t+$ $\Delta t)-x(t) \mid<r)$ para um $t$ arbitrário.

A fim de demonstrar os conceitos previamente discutidos e relacionados à avaliação de estacionariedade, são aplicados os testes STP e ACF sobre as cinco séries temporais definidas na Seção 3.2.

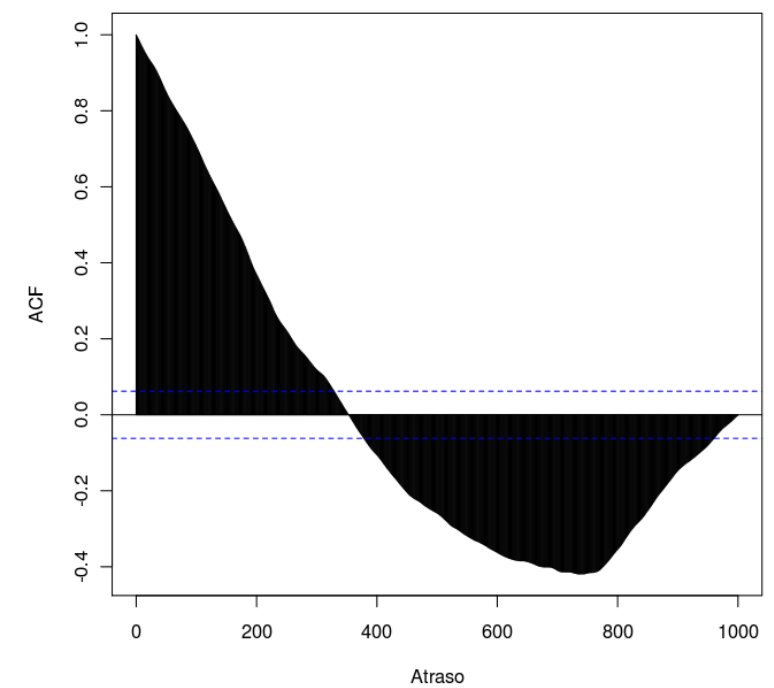

(a) Autocorrelação

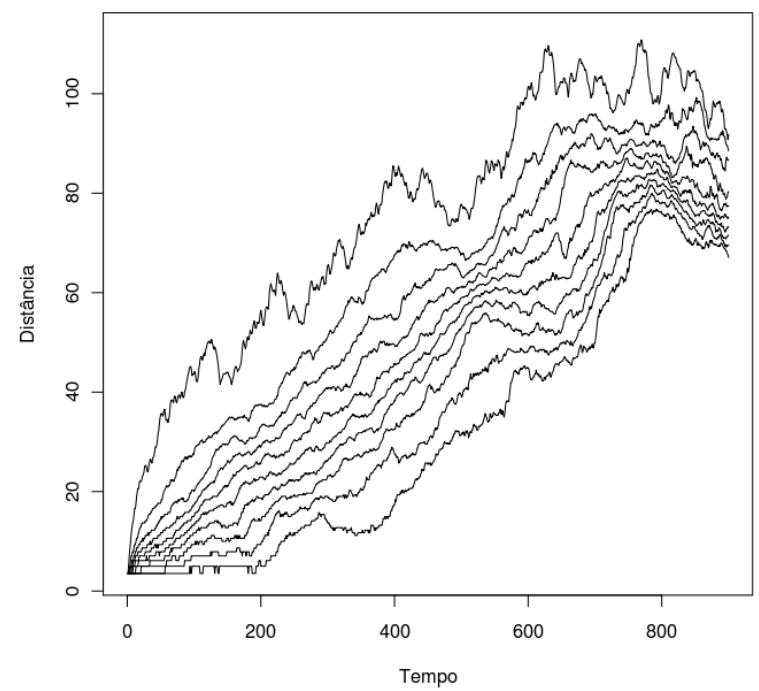

(b) STP

Figura 3.13: Teste de estacionariedade para série temporal Caminhada Aleatória.

A Figura 3.13 apresenta o teste de estacionariedade para a série Caminhada Aleatória. A Figura 3.13(a) apresenta a função de autocorrelação 
dessa série versus o atraso no tempo (ou lag). Nesse tipo de gráfico, se os valores são próximos de zero, para todo atraso no tempo, tem-se um processo aleatório (mas não necessariamente estacionário). Entretanto, processos não aleatórios apresentam valores de autocorrelação, significativamente, diferentes de zero.

Além da aleatoriedade, pode-se aplicar o STP sob uma série a fim de identificar sua estacionariedade. A Figura 3.13(b) apresenta o STP para essa série, onde o eixo das abscissas representa a separação no tempo e o eixo das ordenadas, a separação no espaço. Cada contorno representa uma probabilidade $p$ que revela a periodicidade da série. Na Figura 3.13(b), a separação no tempo demonstra-se pouco correlacionada e a distância aproximada tem variação entre $[1: 100]$ unidades no espaço. Considerando qualquer estado no tempo, tem-se um alto valor no espaço, para qualquer probabilidade $p$, o que caracteriza uma série não estacionária.

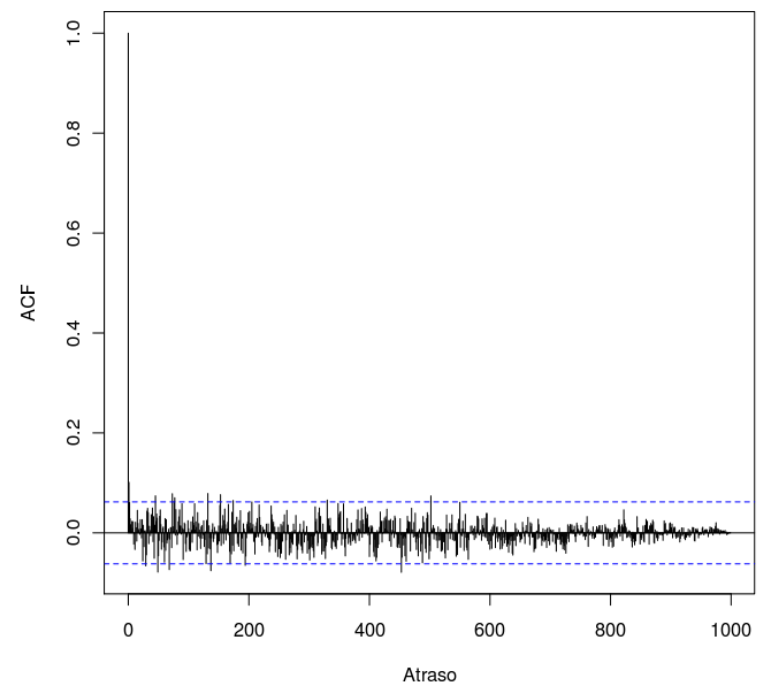

(a) Autocorrelação

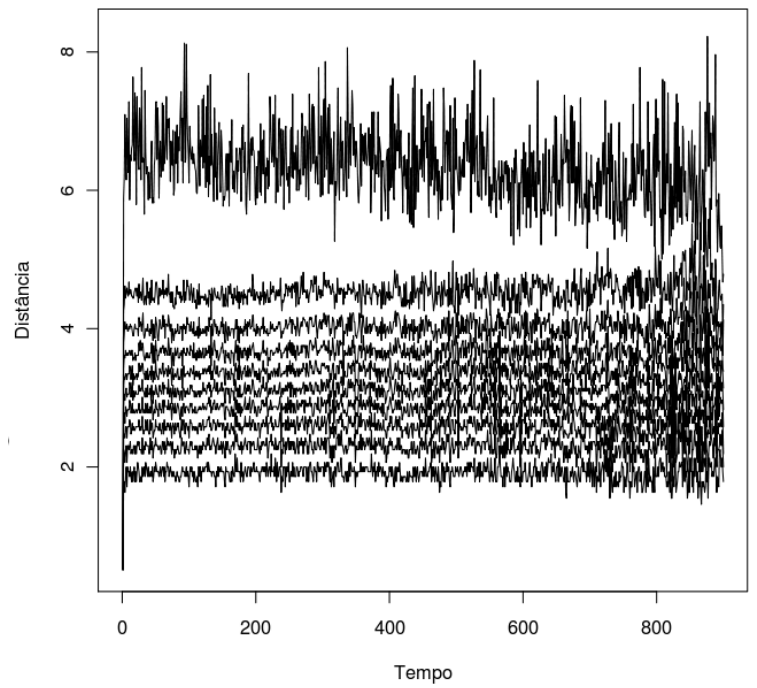

(b) STP

Figura 3.14: Teste de estacionariedade para série temporal AR(1).

A Figura 3.14 apresenta o teste de estacionariedade para a série AR(1). Na Figura 3.14(a), os valores da ACF orbitam em torno de 0. A Figura 3.14(b) apresenta o STP para essa série, onde a separação no tempo apresenta alta correlação e a distância aproximada tem variação entre [1:8] unidades no espaço. Essa série tem valores baixos para quaisquer estados no tempo e quaisquer probabilidades $p$, o que caracteriza uma série estacionária.

A Figura 3.15 apresenta o teste de estacionariedade para a série ARIMA(1,1,1). Segundo a Figura 3.15(a), os valores da ACF são significativamente diferentes de zero. A Figura 3.15(b) apresenta o STP para essa série, onde a separação no espaço tem distância aproximada com variação entre [1 : 15]. Tomando-se quaisquer valores no tempo, tem-se, 


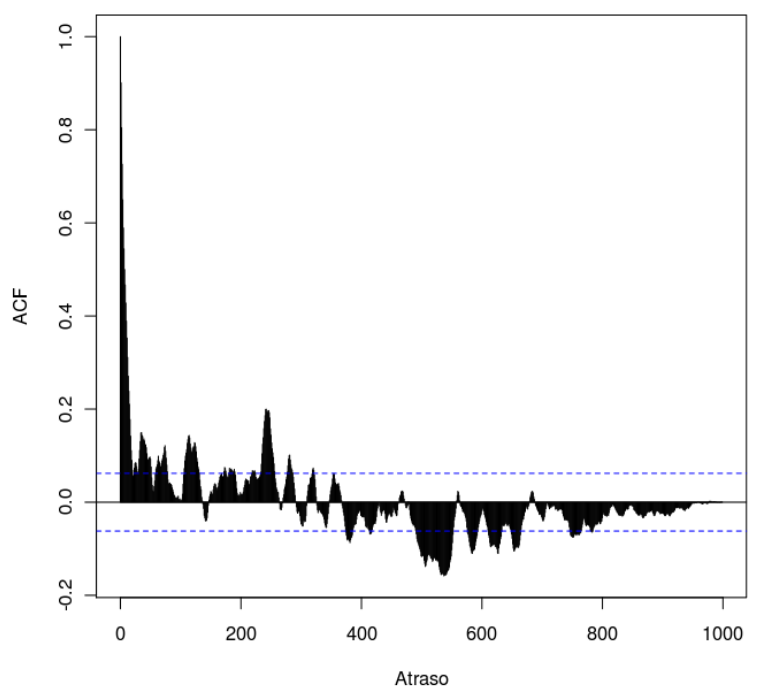

(a) Autocorrelação

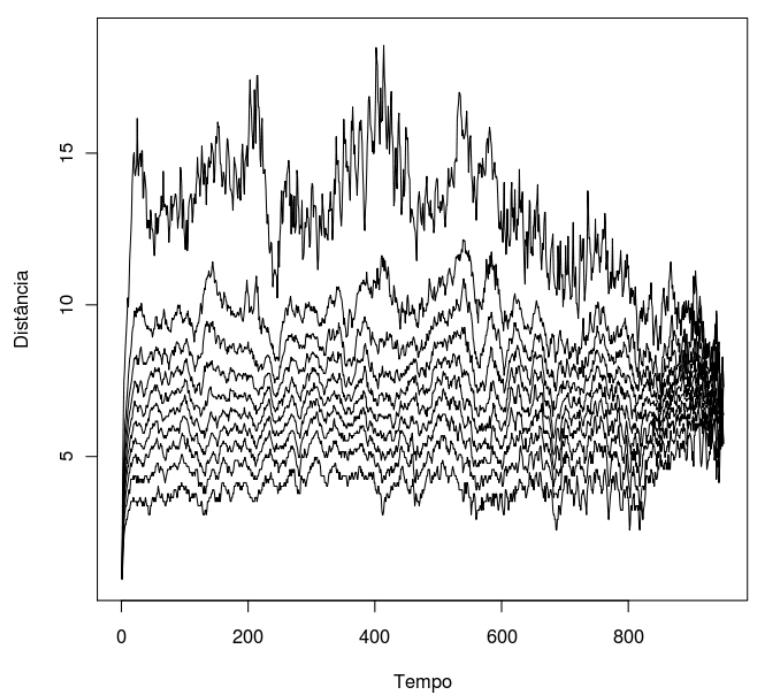

(b) STP

Figura 3.15: Teste de estacionariedade para série temporal $\operatorname{ARIMA}(1,1,1)$.

sempre, altos valores no espaço, o que caracteriza uma série não estacionária.

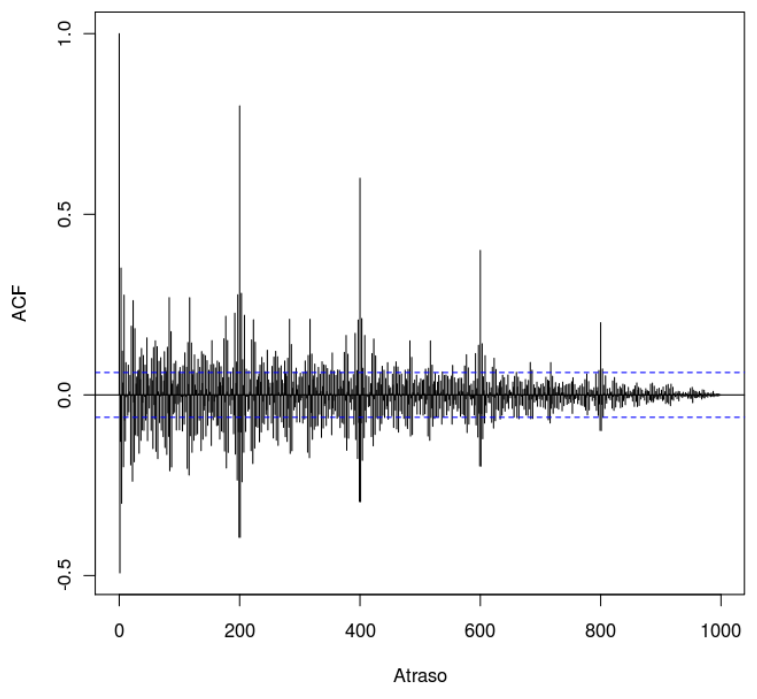

(a) Autocorrelação

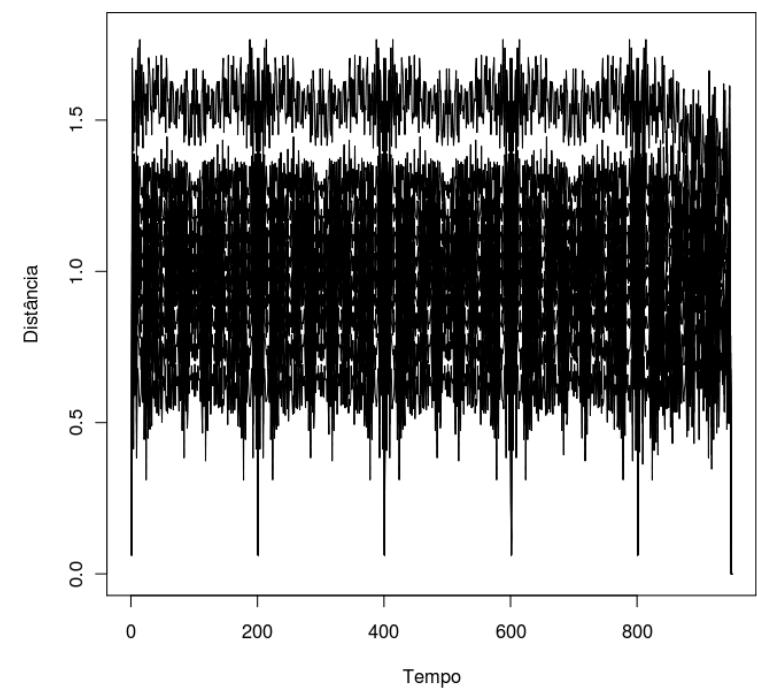

(b) STP

Figura 3.16: Teste de estacionariedade para o mapa Logístico.

A Figura 3.16 apresenta o teste de estacionariedade para a série com observações extraídas de um mapa Logístico. A Figura 3.16(a) apresenta o ACF cujos valores das autocorrelações são próximos de zero. A Figura 3.16(b) apresenta o STP para essa série, onde a separação no espaço tem distância aproximada com variação entre $[1: 1,5]$. Tomando-se quaisquer valores no tempo, tem-se baixos valores no espaço, o que caracteriza uma série estacionária.

A Figura 3.17 apresenta o teste de estacionariedade para a série temporal cujas observações foram extraídas do atrator de Lorenz. Na Figura 3.17(a) 


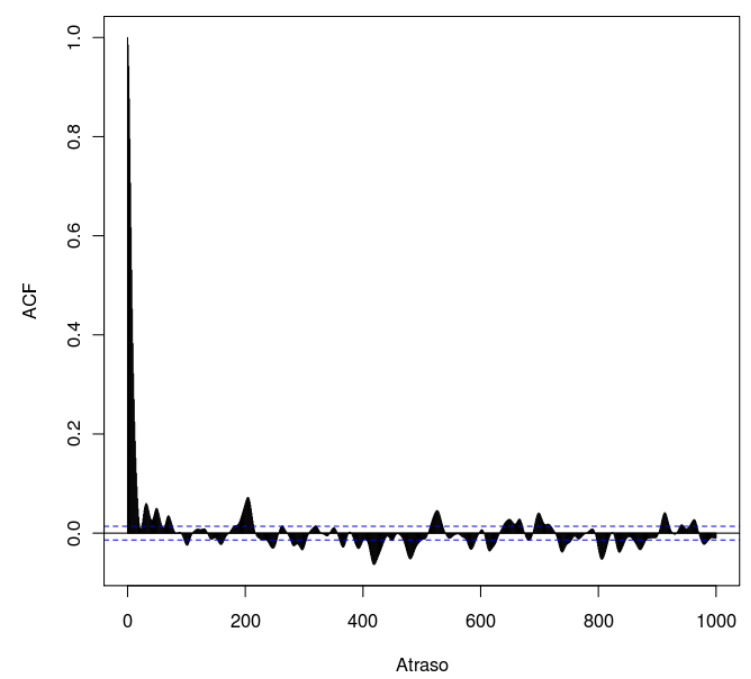

(a) Autocorrelação

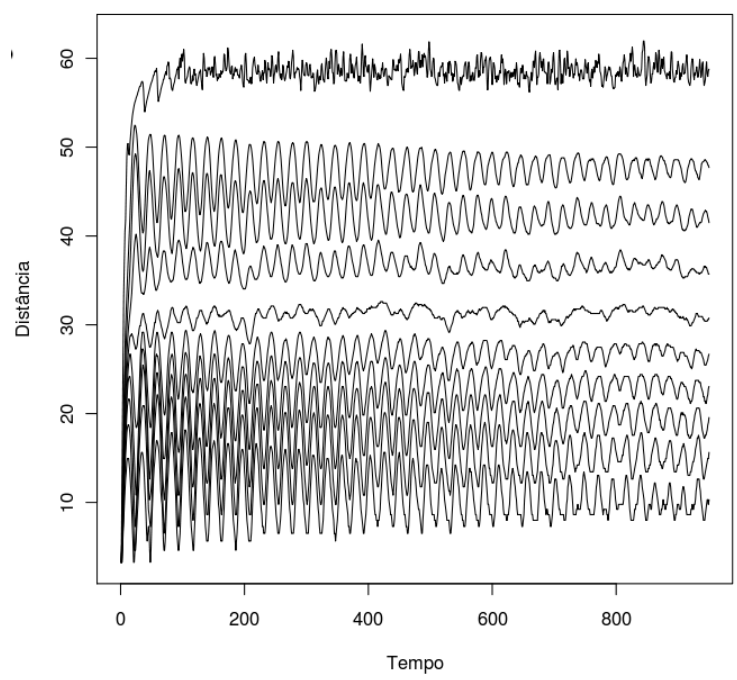

(b) STP

Figura 3.17: Teste de estacionariedade para o atrator de Lorenz.

os valores da ACF são significativamente diferentes de zero. A Figura 3.17(b) apresenta o STP para essa série, onde a separação no espaço tem variação entre [1:60] unidades. Considerando quaisquer valores no tempo, obtém-se altos valores no espaço, o que caracteriza uma série não estacionária.

\subsection{Experimentos com dados reais}

Os testes de aderência conduzidos na Seção 3.5 confirmam a hipótese que séries podem ser avaliadas visando a identificação de propriedades essenciais (tais como a estocasticidade, linearidade e estacionariedade) com o objetivo de classificar seus processos geradores. Entretanto, esses testes foram realizados sobre séries sintéticas e, portanto, outras avaliações são necessárias para sua adoção em situações reais.

A metodologia de classificação de séries temporais, proposta nesta tese, visa avaliar características dos processos geradores de séries temporais, o que pode aumentar a acurácia da modelagem. A fim de validar essa metodologia, experimentos sobre conjuntos de dados reais, obtidos a partir do SNIA (Narayanan et al., 2008) foram realizados. Esses conjuntos de dados foram gerados a partir de servidores de dados (Data Centers da Microsoft Research Cambridge) durante uma semana de monitoração. Esse sistema é constituído por 13 servidores, 36 volumes lógicos e 179 discos rígidos. A Tabela 3.12 descreve o sistema monitorado.

O processo de monitoramento foi realizado em cada volume lógico e foram capturadas todas as operações de leitura e escrita em termos de blocos transferidos. Os traços de execução foram coletados por meio da ferramenta Event Tracing for Windows (ETW), durante 168 horas (uma semana). Cada 
Tabela 3.12: Característica do sistema utilizado no experimentos. Um Data Center com: 13 servidores, 36 volumes lógicos e 179 discos rígidos.

\begin{tabular}{l|lc} 
Servidor & Função & Número de Volumes \\
\hline \hline usr & Diretórios dos usuários & 3 \\
proj & Diretórios de projetos & $\mathbf{5}$ \\
prn & Servidor de impressão & 2 \\
hm & Monitoramento de hardware & $\mathbf{2}$ \\
rsrch & Projetos de pesquisa & 3 \\
prxy & Firewall/web proxy & 2 \\
src1 & Configuração de software & 3 \\
src2 & Configuração de software & 3 \\
stg & Servidor p/ testes de páginas Web & 2 \\
ts & Servidor de terminal & 1 \\
web & Servidor Web/SQL & 4 \\
mds & Servidor Multimidia & $\mathbf{2}$ \\
wdev & Servidor p/ testes de servidores Web & 4 \\
\hline
\end{tabular}

evento capturado descreve uma requisição de E/S (entrada/saída), que inclui o instante, número do disco, primeiro número do bloco lógico, quantidade de blocos transferidos e tipo da operação (leitura ou escrita). Entretanto, de acordo com os propósitos deste trabalho, tais informações foram filtradas e considerou-se, apenas, o instante, a quantidade de blocos transferidos e o tipo da operação. Esses traços de execução são representativos para um grande número de servidores de dados, desde micro e pequenas empresas até empresas médias (Narayanan et al., 2008).

Por razões de simplicidade, espaço e pela maioria dos traços de execução terem comportamentos similares, foram considerados três conjuntos de dados (volumes) para experimentos (linhas em negrito da Tabela 3.12): 1 volume proj com operações somente de escrita, 1 volume hm com operações de leitura e 1 volume mds com operações de leitura. Esses traços foram selecionados para atender cada conjunto de técnicas de predição (ramo da árvore) definida na Figura 3.2.

A metodologia proposta visa classificar traços de execução, considerando a estocasticidade, linearidade e estacionariedade. Assim, essas características foram avaliadas por meio das abordagens descritas na Seção 3.5, onde o RP e as medidas RQA são utilizados para avaliar a estocasticidade (Subseção 3.5.1), WNN avalia a linearidade (Subseção 3.5.2) e STP/ACF verificam a estacionariedade (Subseção 3.5.3).

O primeiro traço avaliado é o proj, cujas operações são somente de escrita. As medidas RQA de proj estão sumarizadas na Tabela 3.13. A partir dos resultados da Tabela 3.13, nota-se que essa série tem baixa taxa de estados recorrentes, estrutura de diagonal com baixa divergência, complexidade e determinismo. Além disso, ao analisar o gráfico RP dessa série (Figura 3.18), 
confirma-se a baixa frequência de diagonais. Dadas essas avaliações, proj pode ser classificada como uma série estocástica ${ }^{8}$.

Tabela 3.13: Medidas RQA para a série proj com dimensão de separação 6, embutida 14 e $\varepsilon=1,0$.

$$
\begin{aligned}
& \text { RR: } 0,3749 \\
& \mathrm{DET}=0,6282 \\
& L_{\text {max }}=21 \\
& L_{\text {mean }}=2,628 \\
& \mathrm{ENTR}=1,082 \\
& \mathrm{DIV}=0,04762
\end{aligned}
$$

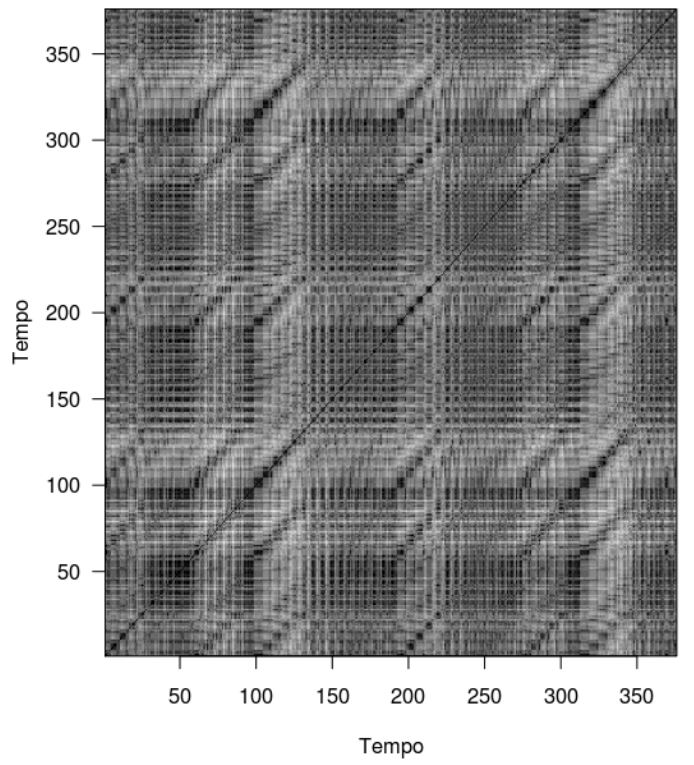

Figura 3.18: Gráfico RP da série proj.

A Tabela 3.14 apresenta medidas RQA para a série hm, que considera operações somente de leitura. A partir dos resultados da Tabela 3.14, nota-se que hm tem baixa quantidade de estados recorrentes. Medidas de estrutura diagonal confirmam que essa série tem divergência moderada, baixa complexidade e grau de determinismo. Além disso, ao analisar seu gráfico RP (Figura 3.19), verifica-se a baixa frequência de linhas diagonais. Ao considerar todas essas avaliações, hm pode ser classificada como estocástica.

Tabela 3.14: Medidas RQA para a série hm com dimensão de separação 4 , embutida 2 $\mathrm{e} \varepsilon=1,0$.

$$
\begin{aligned}
& \text { RR: } 0,19511 \\
& \text { DET }=0,610596 \\
& L_{\text {max }}=3,1275 \\
& L_{\text {mean }}=1,39192 \\
& \mathrm{ENTR}=0,04 \\
& \mathrm{DIV}=26
\end{aligned}
$$

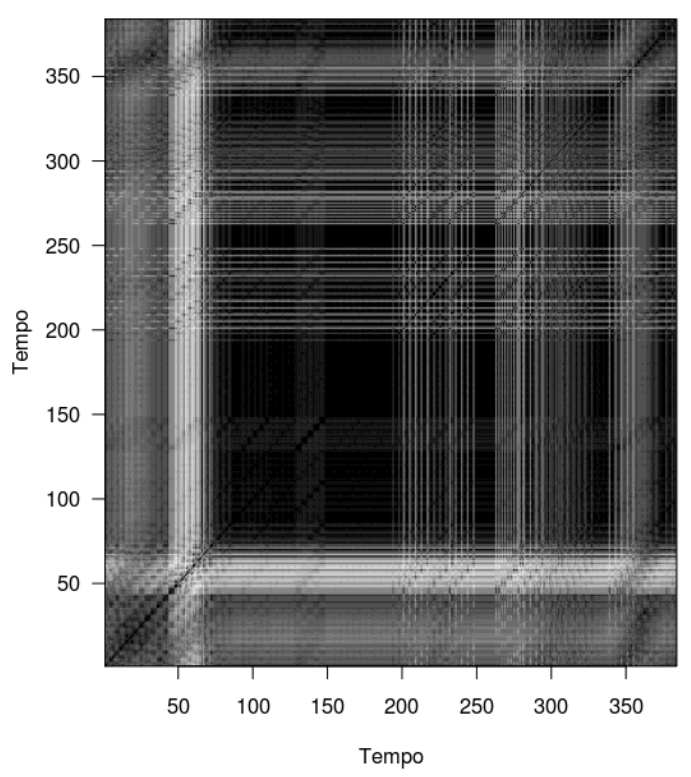

Figura 3.19: Gráfico RP da série hm.

A Tabela 3.15 apresenta medidas RQA para a série mds, que considera

\footnotetext{
${ }^{8}$ Assume-se, no contexto desta tese, que uma série é determinística somente se DET $\geq 0,95$ ou $95 \%$, caso contrário, ela tende a comportamento estocástico.
} 
operações somente de leitura. A partir dos resultados da Tabela 3.15, nota-se que mds tem alta quantidade de estados recorrentes. Medidas de estrutura diagonal confirmam que essa série tem baixa divergência, complexidade moderada e alto grau de determinismo. Além disso, ao analisar seu gráfico RP (Figura 3.20), verifica-se a alta frequência de linhas diagonais. Dadas essas circunstâncias, mds pode ser classificada como uma série determinística.

Tabela 3.15: Medidas RQA para a série mds com dimensão de separação 5 , embutida 2 e $\varepsilon=1,0$.

\begin{tabular}{l}
\hline \hline RR: 0,3749 \\
DET $=0,6282$ \\
$L_{\text {max }}=21$ \\
$L_{\text {mean }}=2,628$ \\
ENTR $=1,082$ \\
DIV $=0,04762$ \\
\hline
\end{tabular}

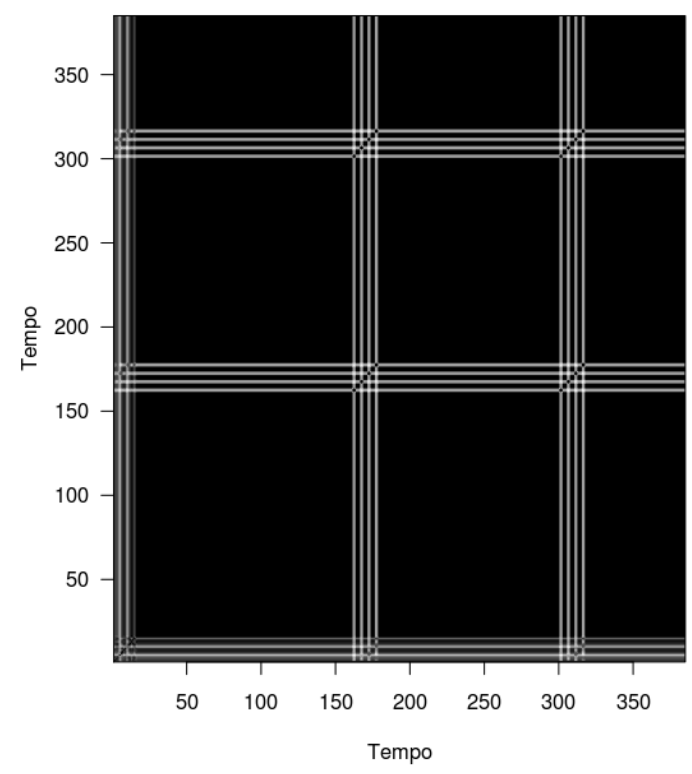

Figura 3.20: Gráfico RP da série mds.

Tabela 3.16: Resultados da aplicação do teste de linearidade WNN sobre três séries temporais obtidas a partir de traços de execução do repositório SNIA.

\begin{tabular}{c|ccc}
\hline Série temporal & $\rho$-value(WNN) & $H_{0}$ & Linearidade \\
\hline \hline proj & 0,05205 & Rejeita & Linear \\
hm & 0,5637 & Rejeita & Linear \\
mds & $9,498 e-07$ & Aceita & Não linear \\
\hline
\end{tabular}

A próxima etapa da metodologia consiste na aplicação do teste WNN (descrito na Subseção 3.3.2) a fim de avaliar os graus de linearidade de todas as séries. A Tabela 3.16 sumariza os resultados dos testes WNN e indica uma classificação para cada série. Neste caso, duas séries são lineares e uma, não linear.

Após a aplicação do teste de linearidade, busca-se identificar os graus de estacionariedade para cada série temporal. Para tanto, os testes STP e ACF, descritos na Seção 3.2, são aplicados sobre as séries proj, hm e mds.

A Figura 3.21 apresenta um teste de estacionariedade para a série proj. Na Figura 3.21(a), os valores da ACF são significativamente próximos de zero. A Figura 3.21(b) apresenta o STP para a mesma série, onde a separação no espaço tem distância aproximada com baixa variação em todos os contornos (cada linha representa um contorno no gráfico da Figura 3.21(b)). Tomando-se 


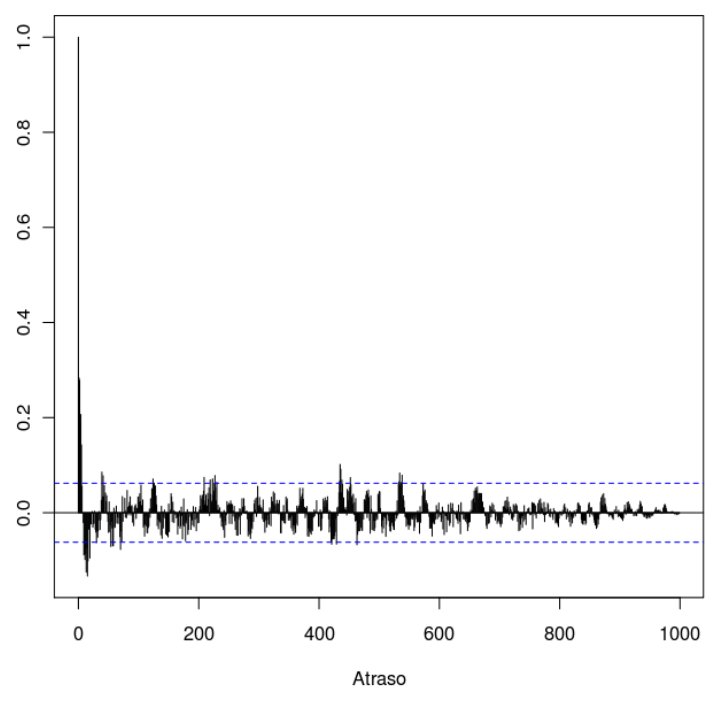

(a) Autocorrelação

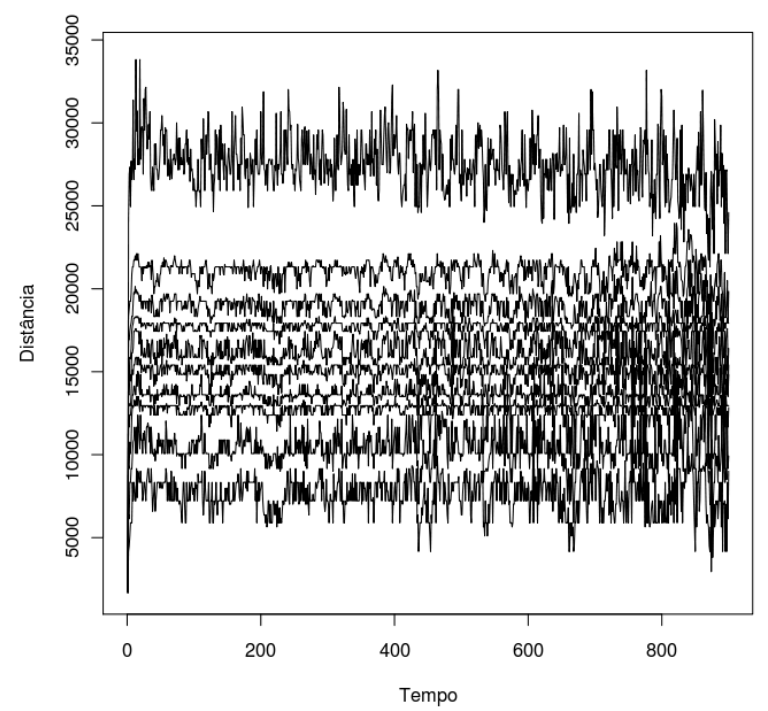

(b) STP

Figura 3.21: Teste de estacionariedade para a série proj.

quaisquer valores no tempo, tem-se poucas variações no espaço, o que caracteriza uma série estacionária.

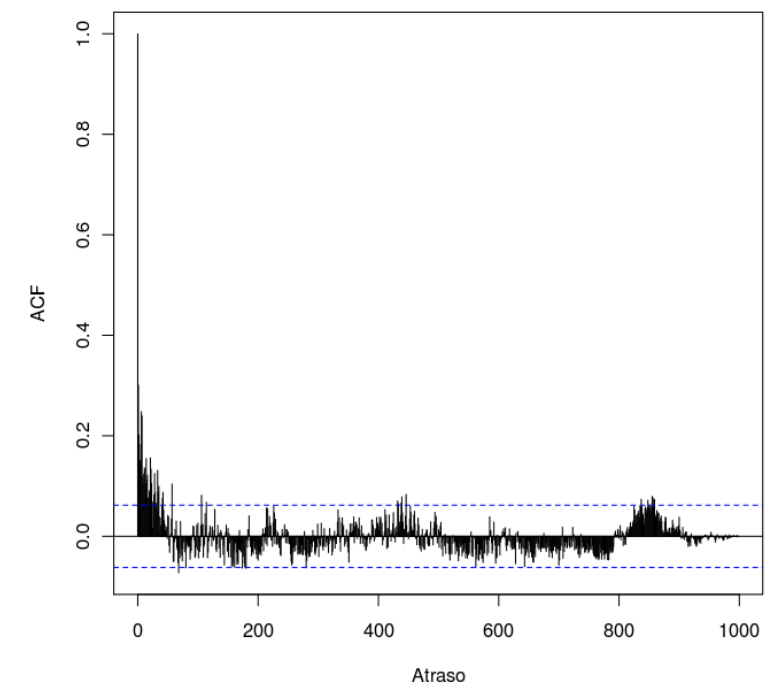

(a) Autocorrelação

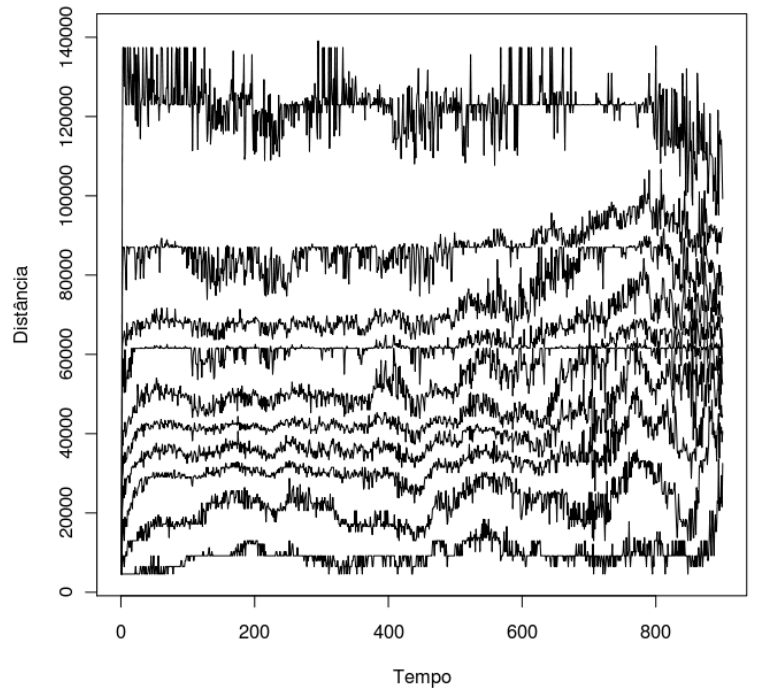

(b) STP

Figura 3.22: Teste de estacionariedade para a série $\mathbf{h m}$.

A Figura 3.22 apresenta um teste de estacionariedade para a série $\mathbf{h m}$. Na Figura 3.22(a), os valores da ACF são significativamente diferentes de zero. A Figura 3.22(b) apresenta o STP para essa série, onde a separação no espaço tem distância com variação acentuada em todos os contornos. Tomando-se quaisquer valores no tempo, tem-se altos valores no espaço, o que caracteriza uma série não estacionária.

A Figura 3.23 apresenta um teste de estacionariedade para a série mds. Na Figura 3.23(a), os valores da ACF apresentam comportamento explosivo 


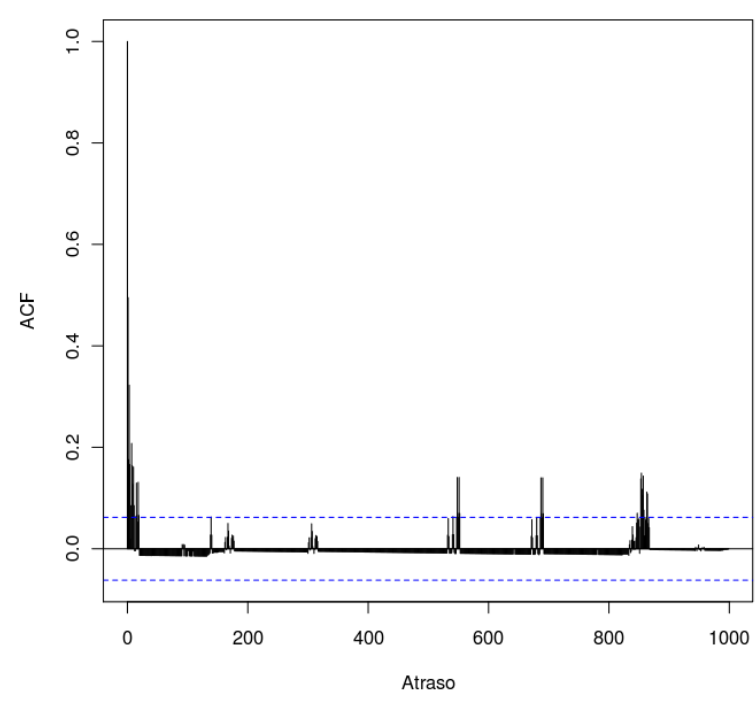

(a) Autocorrelação

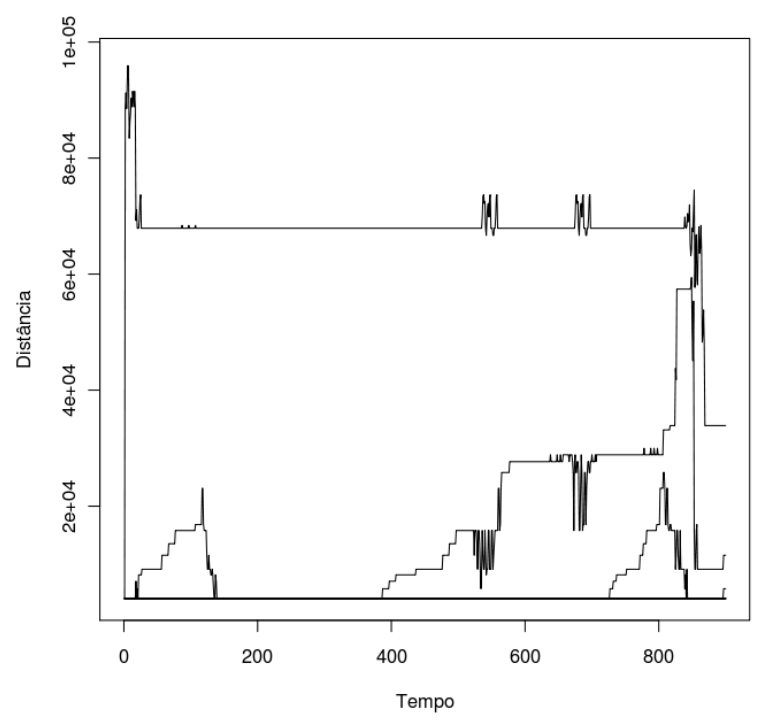

(b) STP

Figura 3.23: Teste de estacionariedade para a série mds.

(presença de picos). A Figura 3.23(b) apresenta o STP para essa série, onde a separação no espaço tem distância com variação acentuada em todos os contornos. Tomando-se quaisquer valores no tempo, tem-se altos valores no espaço, o que caracteriza uma série não estacionária.

\subsection{Avaliação da modelagem}

A metodologia proposta neste capítulo visa a investigação e a classificação de processos geradores de séries temporais. Isso permite que informações relevantes sobre características específicas tais como estocasticidade, linearidade e estacionariedade, contribuam para escolha de técnicas de modelagem mais eficientes e precisas. A Seção 3.6 apresenta resultados que validam a classificação de processos geradores a partir de conjuntos de dados reais. No entanto, a fim de confirmar essa metodologia de classificação, o modelo indicado é submetido à predição de comportamento de processos e sua acurácia, avaliada.

Um conjunto de técnicas de modelagem foram avaliadas considerando séries reais (obtidas a partir de traços de execução do repositório SNIA), a fim de validar a classificação proposta na Figura 3.2 e descrita na Seção 3.4. Para isso, cada série temporal foi subdividida em duas partes: $80 \%$ das observações iniciais são destinadas ao treinamento da abordagem de predição e os $20 \%$ restantes são utilizados para validação. A métrica empregada para a avaliação dos resultados de predição é a Normalized Root Mean Squared Error (NRMSE), definida na Equação 3.31, onde $x_{\max }$ e $x_{\min }$ são valores de observações máximo e mínimo, respectivamente. NRMSE depende da Equação 3.32, que define o 
Root Mean Squared Error (RMSE) (Anderson e Woessner, 1992)), onde $x_{i}$ é o valor esperado no instante $i, \hat{x}_{i}$ é o valor obtido no instante $i$ e $n$ a quantidade de observações preditas.

$$
\begin{aligned}
\text { NRMSE } & =\frac{\text { RMSE }}{x_{\max }-x_{\min }} \\
\text { RMSE } & =\sqrt{\frac{\sum_{i=1}^{n}\left(\hat{x}_{i}-x_{i}\right)^{2}}{n}}
\end{aligned}
$$

A série temporal proj foi a primeira avaliada. Nos testes conduzidos sobre essa série (Seção 3.6), ela é classificada como estocástica, linear e estacionária. Ao considerar essa classificação e de acordo com a abordagem proposta (apresentada na Seção 3.4), essa série pode ser modelada adequadamente por ferramentas estatísticas, tais como processos AR e ARMA (discutidos na Seção 3.3). A Figura 3.24 apresenta os resultados que confirmam essa hipótese, ou seja, essa série modelada por meio de ferramentas estatísticas obtém resultados satisfatórios. Neste sentido, $\operatorname{ARMA}(1,2)$ foi capaz de modelar a série proj com menor erro dentre todas as técnicas avaliadas, indicando uma predição adequada. Para a obtenção das ordens do modelo $\operatorname{ARMA}(1,2)$ foi adotado o pacote auto.arima incluso no software $R^{9}$. Esse pacote encontra a ordem do modelo que melhor se ajusta frente as observações de uma série temporal.

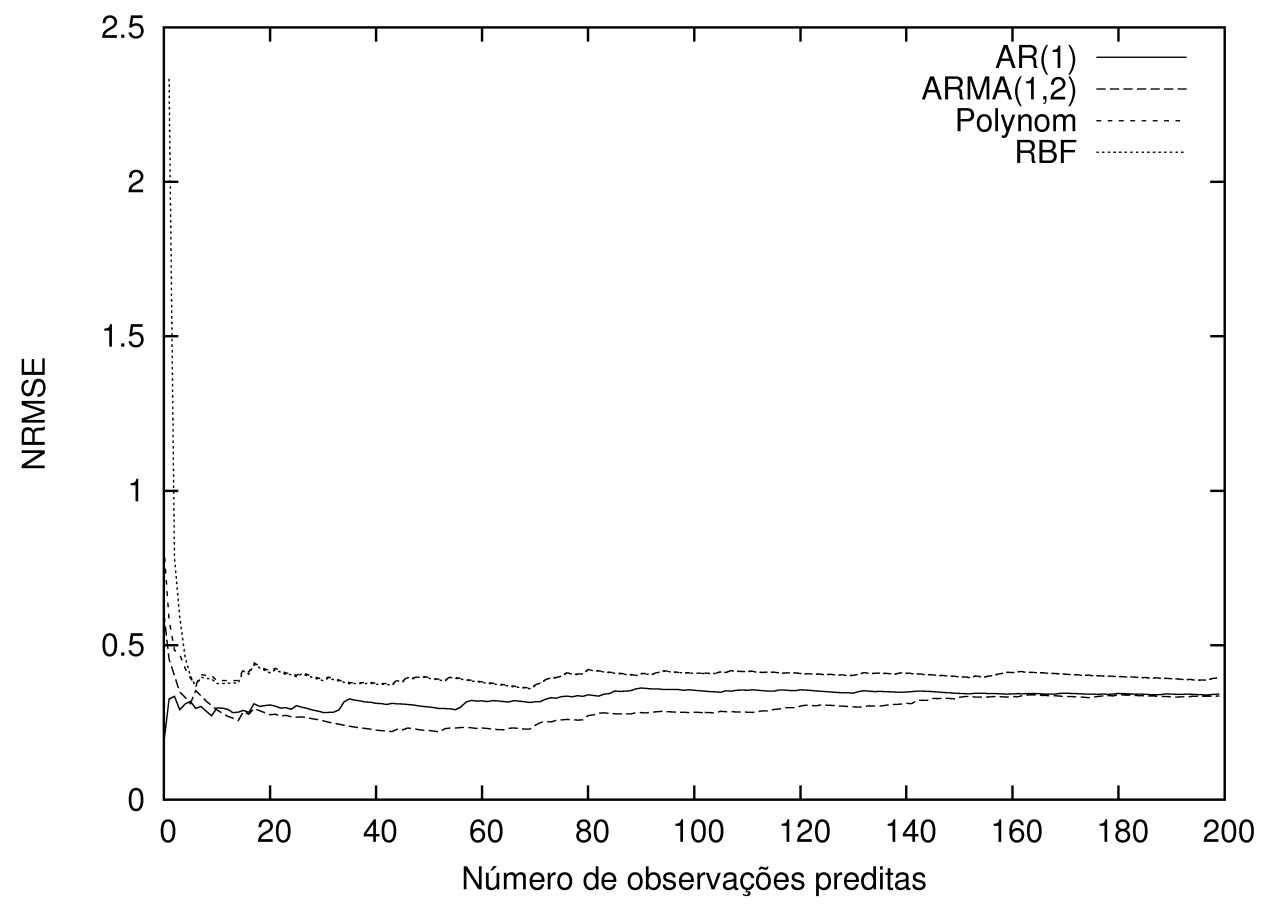

Figura 3.24: Série temporal proj: resultados da métrica NRMSE.

\footnotetext{
${ }^{9}$ http://www.r-project.org/
} 
Além disso, foram conduzidos testes estatísticos sobre todos os resultados de predição. Testes de hipótese foram realizados a fim de confirmar se $\operatorname{ARMA}(1,2)$ é, estatisticamente, melhor que as outras técnicas, ou seja, $\mu\left(\operatorname{NRMSE}_{\mathrm{ARMA}(1,2)}\right)<\mu\left(\mathrm{NRMSE}_{\mathrm{AR}(1)}\right)$ e $\mu\left(\operatorname{NRMSE}_{\mathrm{ARMA}(1,2)}\right)<\mu\left(\mathrm{NRMSE}_{\text {Polynom }}\right)$ e $\mu\left(\mathrm{NRMSE}_{\mathrm{ARMA}(1,2)}\right)<\mu\left(\mathrm{NRMSE}_{\mathrm{RBF}}\right)$.

A fim de comparar $\operatorname{ARMA}(1,2)$ com $\operatorname{AR}(1)$, foram definidas as seguintes hipóteses:

$$
\begin{cases}H_{0}: & \mu\left(\mathrm{NRMSE}_{\mathrm{ARMA}(1,2)}\right)=\mu\left(\mathrm{NRMSE}_{\mathrm{AR}(1)}\right) \\ H_{a}: & \mu\left(\mathrm{NRMSE}_{\mathrm{ARMA}(1,2)}\right)<\mu\left(\mathrm{NRMSE}_{\mathrm{AR}(1)}\right)\end{cases}
$$

A Tabela 3.17 apresenta as médias $(\mu)$ e os desvios padrão $(\sigma)$, em termos de NRMSE, para $\operatorname{AR}(1)$ e $\operatorname{ARMA}(1,2)$. A estatística $z$ foi adotada devido à independência das amostras de dados (Schefler, 1988).

Tabela 3.17: $\mu$ e $\sigma$ de NRMSE para técnica ARMA(1,2) e AR(1).

\begin{tabular}{ccc}
\hline & ARMA(1,2) & AR(1) \\
\hline \hline$\mu$ & 0,2921915 & 0,3308134 \\
$\sigma$ & 0,04644697 & 0,02452782 \\
\hline
\end{tabular}

Ao substituir os valores obtidos da Tabela 3.17 na Equação 3.33, obtém-se:

$$
\begin{gathered}
z=\frac{\mu\left(\operatorname{NRMSE}_{\mathrm{ARMA}(1,2)}\right)-\mu\left(\operatorname{NRMSE}_{\mathrm{AR}(1)}\right)}{\sqrt{\frac{\sigma_{\mathrm{ARMA}(1,2)}^{2}}{n_{\mathrm{ARMA}(1,2)}}+\frac{\sigma_{\mathrm{AR}(1)}^{2}}{n_{\mathrm{AR}(1)}}}} \\
z=\frac{0,2921915-0,3308134}{\sqrt{\frac{0,04644697^{2}}{200}+\frac{0,02452782^{2}}{200}}}=-10,39867
\end{gathered}
$$

Alternativamente, o valor crítico da estatística $z$ é 1,96 para um nível de significância $\alpha=0,05$. Ao aplicar um teste unilateral à esquerda tem-se $\rho$-value $<2,2 e-16$, portanto, pode-se rejeitar $H_{0}$ e, concluir que há evidências suficientes que NRMSE é reduzido ao utilizar a técnica $\operatorname{ARMA}(1,2)$ ao invés de $\operatorname{AR}(1)$.

A fim de comparar $\operatorname{ARMA}(1,2)$ com Polynom, foram definidas as seguintes hipóteses:

$$
\begin{cases}H_{0}: & \mu\left(\operatorname{NRMSE}_{\mathrm{ARMA}(1,2)}\right)=\mu\left(\mathrm{NRMSE}_{\text {Polynom }}\right) \\ H_{a}: & \mu\left(\mathrm{NRMSE}_{\mathrm{ARMA}(1,2)}\right)<\mu\left(\mathrm{NRMSE}_{\text {Polynom }}\right)\end{cases}
$$

A Tabela 3.18 apresenta as médias $(\mu)$ e os desvios padrão $(\sigma)$ da métrica NRMSE para as técnicas ARMA $(1,2)$ e Polynom.

Ao substituir os valores obtidos da Tabela 3.18 na Equação 3.33, obtém-se: 
Tabela 3.18: $\mu$ e $\sigma$ de NRMSE para ARMA(1,2) e Polynom.

$$
\begin{aligned}
& \begin{array}{ccc}
\hline & \text { ARMA(1,2) } & \text { Polynom } \\
\hline \hline \mu & 0,2921915 & 0,404535
\end{array} \\
& \sigma \quad 0,04644697 \quad 0,03574264 \\
& z=\frac{0,2921915-0,404535}{\sqrt{\frac{0,04644697^{2}}{200}+\frac{0,03574264^{2}}{200}}}=-27,10868
\end{aligned}
$$

O teste foi realizado considerando $\alpha=0,05$, assim, o valor crítico da estatística $z$ foi 1,96. Novamente, aplicando-se um teste unilateral à esquerda, tem-se $\rho$-value $<2,2 e-16$, portanto, rejeita-se $H_{0}$ e, conclui-se que há evidências suficientes que $\operatorname{ARMA}(1,2)$ tem menor erro NRMSE que a técnica Polynom.

A fim de comparar $\operatorname{ARMA}(1,2)$ com $\mathrm{RBF}$, foram definidas as seguintes hipóteses:

$$
\begin{cases}H_{0}: & \mu\left(\mathrm{NRMSE}_{\mathrm{ARMA}(1,2)}\right)=\mu\left(\mathrm{NRMSE}_{\mathrm{RBF}}\right) \\ H_{a}: & \mu\left(\mathrm{NRMSE}_{\mathrm{ARMA}(1,2)}\right)<\mu\left(\mathrm{NRMSE}_{\mathrm{RBF}}\right)\end{cases}
$$

A Tabela 3.19 apresenta as médias $(\mu)$ e os desvios padrão $(\sigma)$ da métrica NRMSE para ARMA $(1,2)$ e RBF.

Tabela 3.19: $\mu$ e $\sigma$ de NRMSE para ARMA(1,2) e RBF.

\begin{tabular}{ccc}
\hline & ARMA(1,2) & RBF \\
\hline \hline$\mu$ & 0,2921915 & 0,4117446 \\
$\sigma$ & 0,04644697 & 0,1409526 \\
\hline
\end{tabular}

Ao substituir os valores obtidos da Tabela 3.19 na Equação 3.33, obtém-se:

$$
z=\frac{0,2921915-0,4117446}{\sqrt{\frac{0,04644697^{2}}{200}+\frac{0,1409526^{2}}{200}}}=-11,36674
$$

O teste foi realizado considerando $\alpha=0,05$, assim, o valor crítico da estatística $z$ foi 1,96. Aplicando-se um teste unilateral à esquerda, obtém-se $\rho$-value $<2,2 e-16$, portanto. rejeita-se $H_{0}$ e, conclui-se que há evidências suficientes que $\operatorname{ARMA}(1,2)$ fornece erro menor que a técnica RBF.

Agora, considere a série temporal hm que é classificada como estocástica, linear e não estacionária. De acordo com a classificação proposta, essa série é modelada adequadamente por meio de ferramentas estatísticas, tal como ARIMA. A Figura 3.25 confirma a hipótese de que essa série pode modelada por ferramentas estatísticas. A técnica $\operatorname{ARIMA}(2,1,2)$ foi capaz de modelar a série hm com menor erro. 


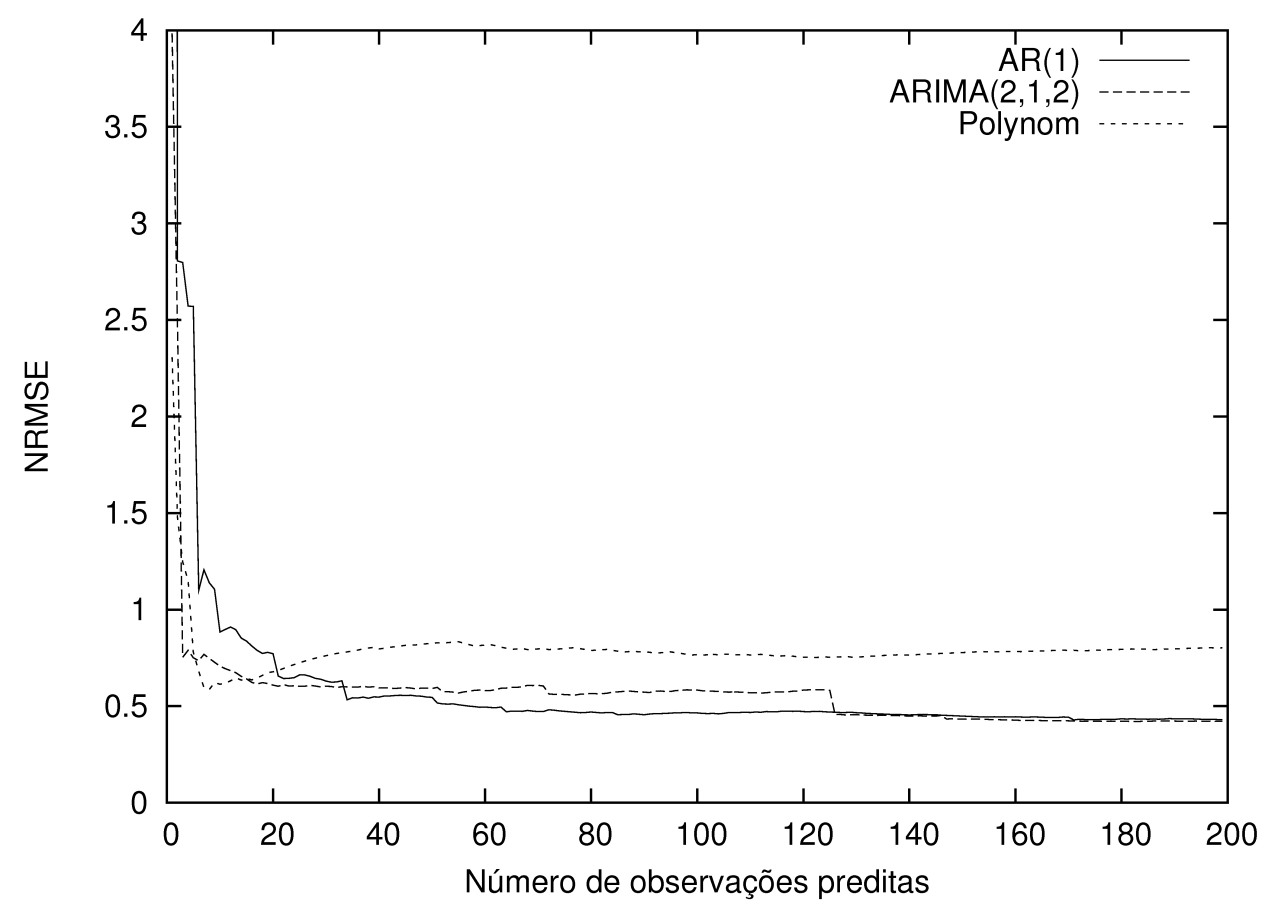

Figura 3.25: Série temporal hm: resultados da métrica NRMSE.

Além disso, foram conduzidos testes estatísticos sobre esses resultados de predição. Um teste de hipótese foi realizado a fim de confirmar se $\operatorname{ARIMA}(2,1,2)$ é estatisticamente melhor que outras técnicas, isto é, $\mu\left(\operatorname{NRMSE}_{\operatorname{ARIMA}(2,1,2)}\right)<$ $\mu\left(\mathrm{NRMSE}_{\mathrm{AR}(1)}\right)$ e $\mu\left(\mathrm{NRMSE}_{\mathrm{ARIMA}(2,1,2)}\right)<\mu\left(\mathrm{NRMSE}_{\text {Polynom }}\right) . \quad$ A técnica $\operatorname{ARIMA}(2,1,2)$ não foi comparada com RBF, pois esta técnica não foi capaz de predizer eventos futuros, mesmo considerando diferentes valores para os centros das funções radiais.

A fim de comparar $\operatorname{ARIMA}(2,1,2)$ com $\operatorname{AR}(1)$, foram definidas as seguintes hipóteses:

$$
\begin{cases}H_{0}: & \mu\left(\mathrm{NRMSE}_{\mathrm{ARIMA}(2,1,2)}\right)=\mu\left(\mathrm{NRMSE}_{\mathrm{AR}(1)}\right) \\ H_{a}: & \mu\left(\mathrm{NRMSE}_{\mathrm{ARIMA}(2,1,2)}\right)<\mu\left(\mathrm{NRMSE}_{\mathrm{AR}(1)}\right)\end{cases}
$$

A Tabela 3.20 apresenta as médias $(\mu)$ e os desvios padrão $(\sigma)$ da métrica NRMSE para ARIMA(2,1,2) e AR(1).

Tabela 3.20: $\mu$ e $\sigma$ de NRMSE para ARIMA(2,1,2) e AR(1).

\begin{tabular}{ccc}
\hline & ARMA(2,1,2) & AR(1) \\
\hline \hline$\mu$ & 0,5636638 & 0,7270487 \\
$\sigma$ & 0,2929577 & 2,399923 \\
\hline
\end{tabular}

Ao substituir os valores obtidos da Tabela 3.20 na Equação 3.33, obtém-se:

$$
z=\frac{0,5636638-0,7270487}{\sqrt{\frac{0,2929577^{2}}{200}+\frac{2,399923^{2}}{200}}}=-0,9532999
$$


O valor crítico considerado para a estatística $z$ foi de 1,96, correspondendo ao nível de significância $\alpha=0,05$. Aplicando-se um teste unilateral à esquerda, obtém-se $\rho$-value $=0.3416$, portanto, aceita-se $H_{0}$ e, conclui-se que $\operatorname{ARIMA}(2,1,2)$ tem erro de predição estatisticamente similar à técnica $\operatorname{AR}(1)$.

A fim de comparar ARIMA $(2,1,2)$ com Polynom, foram definidas a seguintes hipóteses:

$$
\begin{cases}H_{0}: & \mu\left(\mathrm{NRMSE}_{\mathrm{ARIMA}(2,1,2)}\right)=\mu\left(\mathrm{NRMSE}_{\text {Polynom }}\right) \\ H_{a}: & \mu\left(\mathrm{NRMSE}_{\mathrm{ARIMA}(2,1,2)}\right)<\mu\left(\mathrm{NRMSE}_{\text {Polynom }}\right)\end{cases}
$$

A Tabela 3.21 apresenta as médias $(\mu)$ e os desvios padrão $(\sigma)$ da métrica NRMSE para ARIMA $(2,1,2)$ e Polynom.

Tabela 3.21: $\mu$ e $\sigma$ da NRMSE para $\operatorname{ARIMA}(2,1,2)$ e Polynom.

\begin{tabular}{ccc}
\hline & ARIMA(2,1,2) & Polynom \\
\hline \hline$\mu$ & 0,5636638 & 0,7864895 \\
$\sigma$ & 0,2929577 & 0,1344380 \\
\hline
\end{tabular}

Ao substituir os valores obtidos da Tabela 3.21 na Equação 3.33, obtém-se:

$$
z=\frac{0,5636638-0,7864895}{\sqrt{\frac{0,2929577^{2}}{200}+\frac{0,1344380^{2}}{200}}}=-9,7519
$$

Considera-se, novamente, o valor crítico da estatística $z$ como 1,96 e $\alpha=$ 0,05 . Aplica-se um teste unilateral à esquerda e obtém-se $\rho$-value $<2,2 e-16$, portanto, rejeita-se $H_{0}$ e, conclui-se que $\operatorname{ARIMA}(2,1,2)$ tem erro de predição menor que a técnica Polynom.

Finalmente, considere a série temporal mds que é classificada como determinística, não linear e não estacionária. De acordo com a classificação proposta, essa série pode ser modelada adequadamente por ferramentas de sistemas dinâmicos e teoria do caos. A Figura 3.26 confirma essa hipótese, na qual essa série é melhor modelada por ferramentas de teoria do caos (desdobramento de comportamentos no espaço fase e modelagem por meio de RBF). A técnica Espaço Fase/RBF foi capaz de modelar a série mds com menor erro, resultando em predição satisfatória.

Além disso, foram conduzidos testes estatísticos sobre esses resultados de predição. Testes de hipótese foram realizados com objetivo de confirmar se a técnica Espaço Fase/RBF é estatisticamente melhor que outras, ou seja, $\mu\left(\mathrm{NRMSE}_{\text {Phase Space/RBF }}\right)<\mu\left(\mathrm{NRMSE}_{\mathrm{AR}(1)}\right)$ e $\mu\left(\mathrm{NRMSE}_{\text {Phase Space/RBF }}\right)<$ $\mu\left(\mathrm{NRMSE}_{\text {Polynom }}\right)$ e $\mu\left(\mathrm{NRMSE}_{\text {Phase }} \mathrm{Space}_{\mathrm{RBF}}\right)<\mu\left(\mathrm{NRMSE}_{\mathrm{ARMA}(5,5)}\right)$.

A fim de comparar as técnicas Espaço Fase/RBF e AR(1), foram definidas as seguintes hipóteses: 


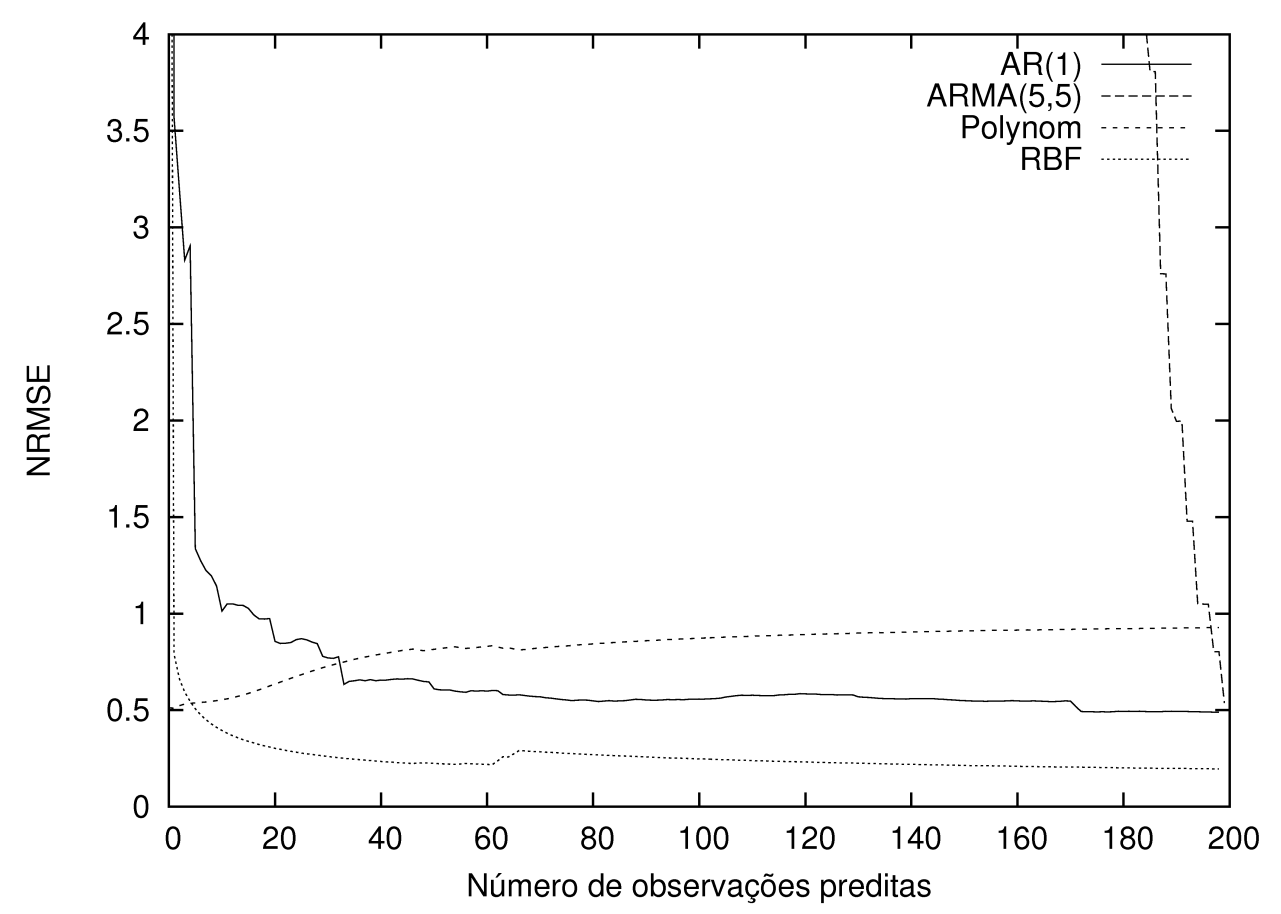

Figura 3.26: Série temporal mds: resultados da métrica NRMSE.

$$
\begin{cases}H_{0}: & \mu\left(\mathrm{NRMSE}_{\text {Phase Space/RBF }}\right)=\mu\left(\mathrm{NRMSE}_{\mathrm{AR}(1)}\right) \\ H_{a}: & \mu\left(\mathrm{NRMSE}_{\text {Phase Space/RBF }}\right)<\mu\left(\mathrm{NRMSE}_{\mathrm{AR}(1)}\right)\end{cases}
$$

A Tabela 3.22 apresenta as médias $(\mu)$ e os desvios padrão $(\sigma)$ da métrica NRMSE para as técnicas Espaço Fase/RBF e AR(1).

Tabela 3.22: $\mu$ e $\sigma$ da NRMSE para as técnicas Espaço Fase/RBF e AR(1).

\begin{tabular}{ccc}
\hline & Espaço Fase/RBF & AR(1) \\
\hline \hline$\mu$ & 0,2979402 & 0,6365301 \\
$\sigma$ & 0,7498916 & 1,175815 \\
\hline
\end{tabular}

Ao substituir os valores obtidos da Tabela 3.22 na Equação 3.33, obtém-se:

$$
z=\frac{0,2979402-0,6365301}{\sqrt{\frac{0,7498916^{2}}{200}+\frac{1,175815^{2}}{200}}}=-4,768364
$$

O valor crítico considerado para a estatística $z$ foi de 1,96 (para $\alpha=0,05$ ). Aplicando-se um teste unilateral à esquerda, obtém-se $\rho$-value $=2,882 e-06$, portanto, rejeita-se $H_{0}$ e, conclui-se que a técnica Espaço Fase/RBF tem erro de predição menor que $\operatorname{AR}(1)$.

A fim de comparar as técnicas Espaço Fase/RBF e Polynom, foram definidas as seguintes hipóteses: 


$$
\begin{cases}H_{0}: & \mu\left(\mathrm{NRMSE}_{\text {Phase Space/RBF }}\right)=\mu\left(\mathrm{NRMSE}_{\text {Polynom }}\right) \\ H_{a}: & \mu\left(\mathrm{NRMSE}_{\text {Phase Space/RBF }}\right)<\mu\left(\mathrm{NRMSE}_{\text {Polynom }}\right)\end{cases}
$$

A Tabela 3.23 apresenta as médias $(\mu)$ e os desvios padrão $(\sigma)$ da métrica NRMSE para as técnicas Espaço Fase/RBF e Polynom.

Tabela 3.23: $\mu$ e $\sigma$ da NRMSE para as técnicas Espaço Fase/RBF e Polynom.

\begin{tabular}{ccc}
\hline & Espaço Fase/RBF & Polynom \\
\hline \hline$\mu$ & 0,2979402 & 0,8305897 \\
$\sigma$ & 0,6365301 & 0,1102992 \\
\hline
\end{tabular}

Ao substituir os valores obtidos da Tabela 3.23 na Equação 3.33, obtém-se:

$$
z=\frac{0,2979402-0,8305897}{\sqrt{\frac{0,6365301^{2}}{200}+\frac{0,1102992^{2}}{200}}}=-11,63121
$$

O valor crítico considerado para a estatística $z$ foi de 1,96 (para $\alpha=0,05$ ). Aplicando-se um teste unilateral à esquerda, obtém-se $\rho$-value $<2,2 e-16$, portanto, rejeita-se $H_{0}$ e conclui-se que a técnica Espaço Fase/RBF tem erro de predição menor que a técnica Polynom.

A fim de comparar as técnicas Espaço Fase/RBF e $\operatorname{ARMA}(5,5)$, foram definidas as seguintes hipóteses:

$$
\begin{cases}H_{0}: & \mu\left(\mathrm{NRMSE}_{\text {Phase Space } / \mathrm{RBF}}\right)=\mu\left(\mathrm{NRMSE}_{\mathrm{ARMA}(5,5)}\right) \\ H_{a}: & \mu\left(\mathrm{NRMSE}_{\text {Phase Space } / \mathrm{RBF}}\right)<\mu\left(\mathrm{NRMSE}_{\mathrm{ARMA}(5,5)}\right)\end{cases}
$$

A Tabela 3.24 apresenta as médias $(\mu)$ e os desvios padrão $(\sigma)$ da métrica NRMSE para as técnicas Espaço Fase/RBF e ARMA(5,5).

Tabela 3.24: $\mu$ e $\sigma$ da NRMSE para as técnicas Espaço Fase/RBF e ARMA(5,5).

\begin{tabular}{ccc}
\hline & Espaço Fase/RBF & ARMA(5,5) \\
\hline \hline$\mu$ & 0,2979402 & $1,817834 e+06$ \\
$\sigma$ & 0,6365301 & 3130255 \\
\hline
\end{tabular}

Ao substituir os valores obtidos da Tabela 3.24 na Equação 3.33, obtém-se:

$$
z=\frac{0,2979402-1,817834 e+06}{\sqrt{\frac{0,6365301^{2}}{200}+\frac{3130255^{2}}{200}}}=-8,192208
$$

O valor crítico considerado para a estatística $z$ foi de 1,96 (assumindo nível de significância $\alpha=0,05$ ). Aplicando-se um teste unilateral à esquerda, obtém-se $\rho$-value $=3.130 e-14$, portanto, rejeita-se $H_{0}$ e, conclui-se que a técnica Espaço Fase/RBF tem erro de predição menor que a técnica $\operatorname{ARMA}(5,5)$. 


\subsection{Considerações Finais}

Este capítulo apresentou uma metodologia para a classificação de processos geradores de séries temporais. Testes de aderência foram utilizados para identificar níveis de estocasticidade, linearidade e estacionariedade. Após a classificação da série, um modelo que melhor se ajusta ao comportamento das observações é obtido. A partir desse modelo, predições podem ser realizadas sobre eventos de leitura e escrita em arquivos. Essa metodologia permite maior acurácia na modelagem e elimina a necessidade de um especialista para analisar as séries temporais em estudo.

Resultados apresentados na Seção 3.7 confirmam que as técnicas de modelagem indicadas pela taxonomia de classificação, proposta na Seção 3.4, têm erro de predição reduzido quando comparadas às demais técnicas. Esses resultados permitem a proposta de abordagens on-line que visam otimizar operações de acesso a dados. Uma abordagem que utiliza essa metodologia é apresentada na Seção 4.5. 


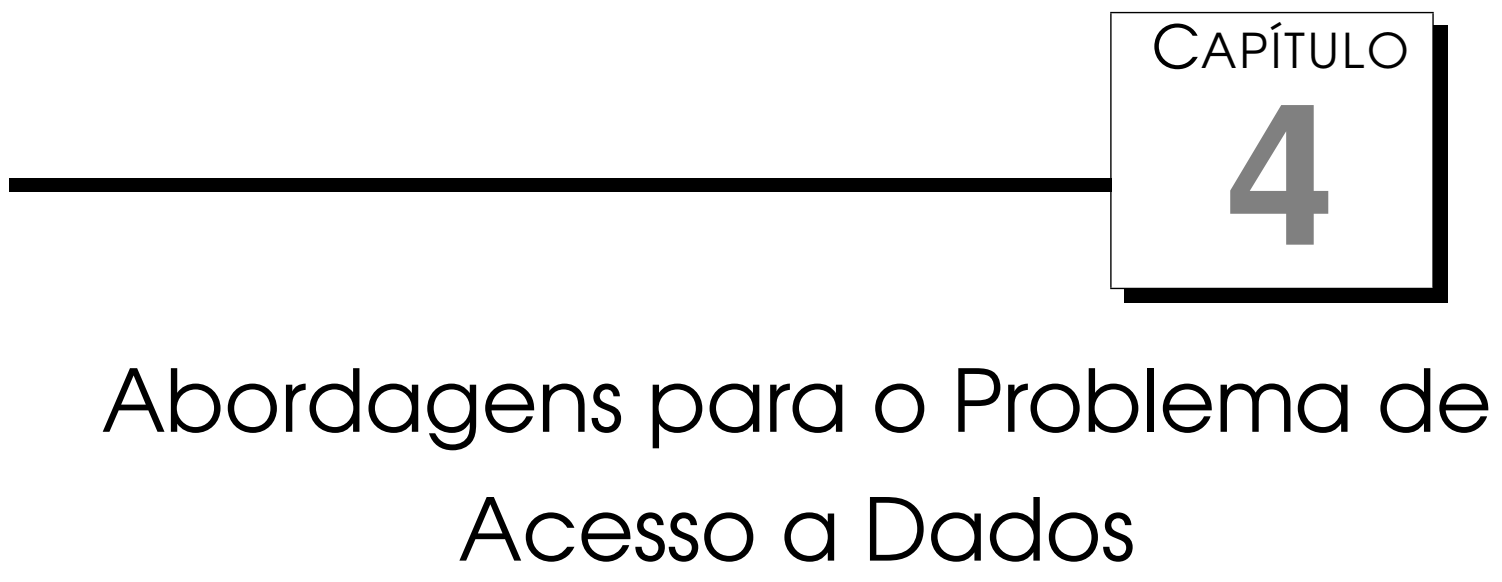

Este capítulo apresenta duas abordagens para solucionar o problema de acesso a dados (PAD). Uma definição empírica desse problema é apresentada na Seção 4.2. A Seção 4.3 define, formalmente, esse problema e demonstra uma prova de sua NP-Completude. Em seguida, na Seção 4.4, é apresentada uma primeira abordagem de otimização de acesso a dados baseada em histórico, a metodologia adotada, experimentos e resultados. Na Seção 4.5 é apresentada a segunda abordagem proposta no contexto desta tese, que emprega predição on-line de comportamento de aplicações como forma de otimizar o acesso a dados distribuídos. Em seguida, é apresentada a metodologia adotada, experimentos e os resultados obtidos.

\subsection{Considerações Iniciais}

O volume de dados gerados por aplicações data-intensive tem aumentado intensamente nas últimas décadas (Cochrane et al., 2009). Mesmo incluindo novos dispositivos de armazenamento, ainda há gargalos, os quais se encontram, principalmente, nas taxas de transferência de dados entre diferentes regiões de ambientes distribuídos. Por outro lado, quando se trata de ambientes distribuídos de larga escala, problemas adicionais surgem tal como a manutenção de redundância (backup). Esses problemas encontram alternativas em técnicas de replicação, migração de dados, acesso paralelo, etc (Gray, 2009).

A necessidade de acesso eficiente por aplicações data-intensive motivou 
esta tese cuja hipótese é: a antecipação de operações de leitura e escrita, por meio de estimativas, predições e análise de séries temporais, pode aumentar o desempenho de aplicações que manipulam grandes volumes de dados.

Para confirmar essa hipótese, são propostas abordagens para otimizar operações de entrada e saída, ou seja, operações de leitura e escrita em arquivos distribuídos. Técnicas atuais (discutidas no Capítulo 2) apresentam limitações, principalmente por não extraírem e utilizarem informações comportamentais de aplicações a fim de otimizar acessos. Neste contexto, projetou-se abordagens que empregam conhecimentos sobre processos para tomada de decisões relativas à replicação, migração e consistência de dados distribuídos.

\subsection{Problema de acesso a dados: Definição Empírica}

A fim de definir empiricamente o problema de acesso a dados (PAD), considere o seguinte estudo de caso hipotético: dadas duas aplicações paralelas compostas por processos descritos na Tabela 4.1, onde MI representa milhões de instruções executadas por um processo $p(p \in P)$; ML e ME são, respectivamente, as quantidades de KBytes/s lidos e escritos da memória principal; HDL e HDE são, respectivamente, as quantidades de KBytes/s lidos e escritos do disco rígido (ou memória secundária); NETR e NETE são, respectivamente, as quantidades de KBytes/s recebidos e enviados através da rede de comunicação - nesse caso, são representados os processos emissor (NETE) e receptor (NETR). Por exemplo, a linha 1 da Tabela 4.1 indica que o processo $p_{0}$ consome $1.234 \mathrm{MI}$, lê $123 \mathrm{KBytes} / \mathrm{s}$ (e não escreve dados na memória, ou seja, $\mathrm{ME}=0$ ), além disso, lê $78 \mathrm{KBytes} / \mathrm{s}$ do disco rígido (e não escreve dados no disco, ou seja, $\mathrm{HDE}=0$ ), recebe $12 \mathrm{KBytes} / \mathrm{s}$ do processo $p_{1} \mathrm{e}$ envia $532 \mathrm{KBytes} / \mathrm{s}$ para o processo $p_{1}$.

Tabela 4.1: Comportamento de Processos

Aplicação 0

\begin{tabular}{|c|c|c|c|c|c|c|c|}
\hline Processo & MI & ML & ME & HDL & HDE & NETR & NETE \\
\hline$p_{0}$ & 1.234 & 123 & 0 & 78 & 0 & $12-p_{1}$ & $532-p_{1}$ \\
\hline$p_{1}$ & 1.537 & 23 & 89 & 0 & 12 & $532-p_{0}$ & $12-p_{0}$ \\
\hline \multicolumn{8}{|c|}{ Aplicação 1} \\
\hline Processo & MI & ML & ME & HDL & HDE & NETR & NETE \\
\hline$p_{2}$ & 1.221 & 823 & 70 & 78 & 543 & $10-p_{3}$ & $321-p_{4}$ \\
\hline$p_{3}$ & 1.137 & 223 & 179 & 324 & 212 & $423-p_{4}$ & $10-p_{2}$ \\
\hline$p_{4}$ & 2.237 & 23 & 17 & 12 & 0 & $321-p_{2}$ & $423-p_{3}$ \\
\hline
\end{tabular}


Agora, considere que os processos descritos na Tabela 4.1 foram alocados em um conjunto de computadores $C$, descritos na Tabela 4.2, onde EP é um elemento de processamento; MIPS representa a capacidade de processamento em milhões de instruções por segundo; TML e TME são, respectivamente, as vazões (throughput) de leitura e escrita na memória principal (em KBytes/s); THDL e THDE são, respectivamente, as vazões de leitura e escrita em disco (também em KBytes/s). Considere, ainda, que $\propto$ é um operador que define a alocação de processos sobre uma infraestrutura computacional. Seja, por exemplo, uma aplicação composta por 5 processos, a alocação poderia ser definida tal como $p_{0} \propto \mathrm{EP}_{0}, p_{1} \propto \mathrm{EP}_{1}, p_{2} \propto \mathrm{EP}_{2}, p_{3} \propto \mathrm{EP}_{3}$ e $p_{4} \propto \mathrm{EP}_{4}$. Considere, também, que os elementos de processamento em $C$ estão interconectados de acordo com a Figura 4.1.

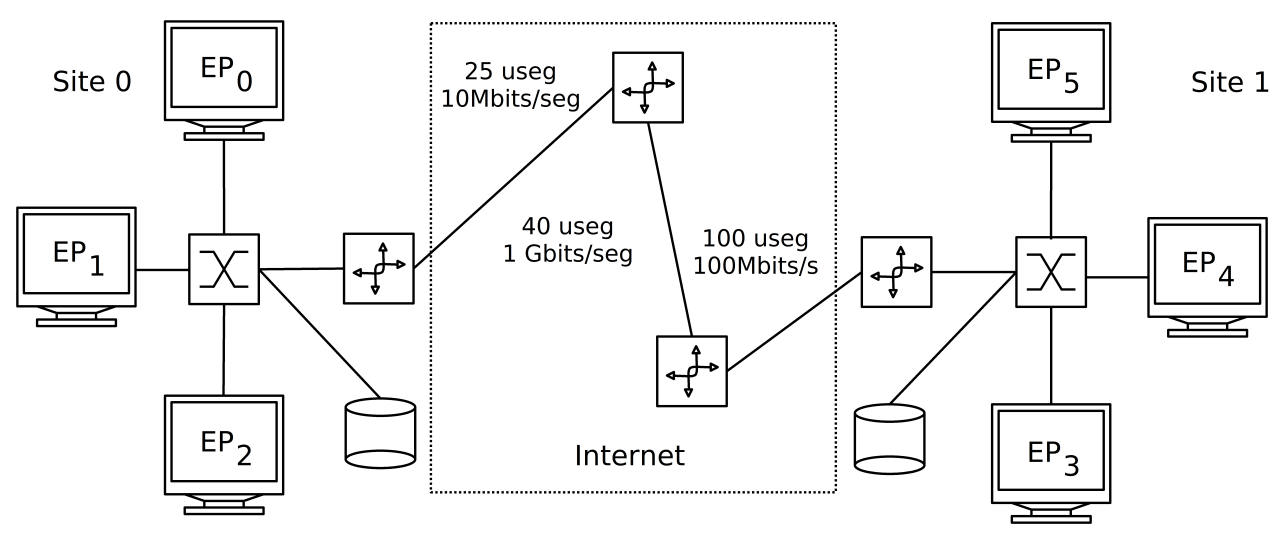

Figura 4.1: Exemplo de um ambiente distribuído com respectivos elementos de processamento e rede de comunicação.

Tabela 4.2: Características dos elementos de processamento da grade apresentada na Figura 4.1 .

\begin{tabular}{lccccc}
\hline & MIPS & TML & TME & THDL & THDE \\
\hline \hline $\mathrm{EP}_{0}$ & 1.200 & 100.000 & 40.000 & 32.000 & 17.000 \\
$\mathrm{EP}_{1}$ & 2.100 & 120.000 & 50.000 & 42.000 & 19.000 \\
$\mathrm{EP}_{2}$ & 1.800 & 100.000 & 30.000 & 22.000 & 9.000 \\
$\mathrm{EP}_{3}$ & 1.700 & 95.000 & 20.000 & 25.000 & 11.000 \\
$\mathrm{EP}_{4}$ & 2.500 & 110.000 & 60.000 & 62.000 & 30.000 \\
$\mathrm{EP}_{5}$ & 2.000 & 110.000 & 45.000 & 40.000 & 17.000 \\
\hline
\end{tabular}

Considere que todos os 5 processos (descritos na Tabela 4.1) das duas aplicações paralelas acessam um conjunto de arquivos $F$ composto por $\left\{f_{0}, f_{1}, \ldots, f_{9}\right\}$. Para resolver o problema de alocação dos arquivos em $F$ sobre os elementos de $C$, é necessário explorar todas as possibilidades de distribuição de arquivos e, além disso, avaliar os custos de acesso para todos 
os processos em $P$. Nessa situação, permutações podem ser realizadas com objetivo de investigar todas as soluções possiveis. Por exemplo, uma solução cujo subconjunto de arquivos $\left\{f_{0}, \ldots, f_{6}\right\}$ está alocado no $\mathrm{EP}_{0}$ e todos os outros $\left(\left\{f_{7}, f_{8}, f_{9}\right\}\right)$ estão alocados no $\mathrm{EP}_{1}$. O conjunto de todas as soluções possíveis, para qualquer instância desse problema, é obtido por meio do cálculo de $n^{z}$, onde $n$ é o número de elementos de processamento e $z$ a quantidade de arquivos. Dessa maneira, considerando a instância previamente mencionada, o espaço de soluções é correspondente a $6^{10}=60.466 .176$.

Além desse exemplo, é necessário investigar o problema em condições reais. Atualmente, aplicações acessam arquivos cujos tamanhos variam na ordem de GBytes a TBytes. Apenas com propósitos ilustrativos, assuma que os trechos dos arquivos tenham em torno de 32 KBytes e que o ambiente alvo contenha mais que 256 elementos de processamento. Nessas condições, o espaço de soluções esperadas pode alcançar ordens de magnitude definidas tal como na Tabela 4.3. Em condições reais, espera-se que a grade seja composta por milhares de computadores armazenando milhões de arquivos, onde cada arquivo apresenta tamanho de GBytes a TBytes.

Tabela 4.3: Espaço de soluções para o problema de distribuição de arquivos sobre os elementos de processamento de uma grade.

\begin{tabular}{ccc}
\hline EP's & $\begin{array}{c}\text { Número } \\
\text { de arquivos }\end{array}$ & Soluções: $n^{z}$ \\
\hline \hline 256 & 320 & $\approx 4 \times 10^{770}$ \\
256 & 3.200 & $\approx 2 \times 10^{7.706}$ \\
256 & 32.768 & $\approx 1 \times 10^{78.913}$ \\
512 & 320 & $\approx 9 \times 10^{866}$ \\
512 & 3.200 & $\approx 4 \times 10^{8.669}$ \\
512 & 32.768 & $\approx 2 \times 10^{88.777}$ \\
1.024 & 320 & $\approx 1 \times 10^{963}$ \\
1.024 & 3.200 & $\approx 9 \times 10^{9.632}$ \\
1.024 & 32.768 & $\approx 3 \times 10^{98.641}$ \\
\hline
\end{tabular}

\subsection{Problema de acesso a dados: Definição Formal}

Um ambiente de grade computacional pode ser representado por um grafo não direcionado $G=(S, L)$, onde o conjunto de vértices $S$ representa regiões, ou sites (um domínio composto por estações de trabalho, máquinas paralelas, 
aglomerados, etc.), e o conjunto de arestas $L$ é constituído por canais de comunicação entre sites. Considere também um conjunto de arquivos $F$ com $z$ elementos, cujo tamanho dos arquivos pode ser modelado por uma função $\theta():. F \rightarrow \mathbb{Z}^{+}$. Cada site $s \in S$ tem um ou mais elementos com capacidade de armazenamento definida por uma função $\Omega: S \rightarrow \mathbb{Z}^{+}$, que representa o tamanho, em MBytes, desses elementos em um site $s$. A função $\alpha_{j, i}: F \times S \rightarrow \mathbb{Z}^{+}$ define o custo de armazenamento do arquivo $j$ no site $s_{i}$. Da mesma maneira, a função $\delta():. F \times S \rightarrow \mathbb{Q}^{+}$considera os canais em $L$, a fim de modelar custos de comunicação entre os sites. A função $\alpha$ tem restrição definida por $\alpha_{j, i} \leq \Omega_{i} \forall j, i$.

Considere um conjunto $A$ constituído por $k$ aplicações executando em um ambiente de larga escala. Seja a função $\phi():. A \rightarrow \mathbb{Z}^{+}$o número de processos para cada aplicação. Considere também o conjunto $P$ composto por todos os processos de todas as $k$ aplicações, ou seja, o número de elementos em $P$ é igual a $h=\sum_{a \in A} \phi(a)$. Todos os processos foram previamente alocados nesse ambiente de acordo com qualquer política de escalonamento ${ }^{1}$.

O custo para transferir um arquivo $f$, do site $s$ para $s^{\prime}$, pode ser representado por meio da Equação 4.1, que depende do tamanho do arquivo $\theta(f)$ e da distância $d\left(s, s^{\prime}\right)$ entre os sites ${ }^{2}$. O custo $\psi$ é atribuído caso exista alguma restrição (segurança, falha, etc.) que impeça a replicação do arquivo $f$ no site $s^{\prime}$.

$$
\delta(s, f)= \begin{cases}\theta\left(f_{j}\right) d\left(s, s^{\prime}\right) & \text { onde } s \in S, j=1, \ldots, z \\ \psi=\infty & \text { caso contrário }\end{cases}
$$

Um arquivo $f$ deve ser transferido a partir do site $s \in S$ que apresente menor caminho, ou seja, o custo de transferência definido na função $d\left(s, s^{\prime}\right)$ é mínimo. O custo de leitura de um arquivo $f$, armazenado em um site s, é portanto, dado pela Equação 4.2. Operações de escrita sobre os dados implicam em atualizações de réplicas para cada arquivo $f \in F$ e o custo pode ser descrito por meio da Equação 4.3.

$$
\begin{gathered}
r_{\text {cost }}(f)=\delta\left(s^{\prime}, f\right)=\arg \min \left(\theta(f) d\left(s, s^{\prime}\right)\right) \forall s \in S \\
w_{\text {cost }}(f)=\sum_{j} \delta\left(s^{\prime}, f_{j}\right) \forall j
\end{gathered}
$$

O custo total de acesso (leitura/escrita) a um arquivo $f$ pode ser determinado por meio da Equação 4.4, que unifica a Equação 4.2 e a

\footnotetext{
${ }^{1} \mathrm{O}$ problema do escalonamento de processos não faz parte do escopo desta tese.

${ }^{2}$ Essa distância é calculada em termos de largura de banda e atraso dos canais de comunicação.
} 
Equação 4.3, considerando todos os processos em P. A Tabela 4.4 descreve todos os parâmetros do modelo proposto.

$$
\Lambda(f)=\sum_{p \in P} \sum_{j} r_{\text {cost }}\left(f_{j}\right)+w_{\text {cost }}\left(f_{j}\right)
$$

Considere um conjunto de processos $P$ previamente alocados sobre um conjunto de sites $S$ com capacidade de armazenamento definido por $\alpha$ e custo de transferência $\delta\left(s, s^{\prime}\right)$. Seja o conjunto de arquivos $F$ com uma dada distribuição inicial $x_{i j}$ e tamanho $\theta(f)$. Assumindo-se um conjunto de quíntuplas TR, que descrevem operações de leitura e escrita sobre os arquivos em $F$, o problema de otimização de acesso a dados, PAD, consiste em determinar uma nova distribuição de arquivos $y_{i j}$ de acordo com a função objetivo definida na Equação 4.5, cujas restrições estão definidas na Equação 4.6 e na Equação 4.7, que apresentam os seguintes domínios: $x_{i j} \in\{0,1\}, \forall i, j$ e $y_{i j} \in\{0,1\} \forall i, j$.

$$
\begin{gathered}
\Gamma(\mathbf{P A D})=\min \sum_{i}^{n} \sum_{j}^{z}\left(x_{i j}-y_{i j}\right) \Lambda\left(f_{j}\right) \\
\sum_{i}^{n} x_{i} f_{j} \leq n \\
\sum_{j}^{z} y_{i j}=1, \quad i=1, \ldots, n
\end{gathered}
$$

A função objetivo (Equação 4.5) visa a redução dos custos de acesso aos arquivos, considerando a distância entre os sites, a quantidade de informação e a capacidade de armazenamento. A restrição definida na Equação 4.6 limita a quantidade de réplicas de um arquivo em $n$ sites, ou seja, se $j=n$ todos os sites da grade tem uma cópia local do arquivo $f$. Na restrição definida na Equação 4.7, toda réplica deve estar alocada somente em um único site, ou seja, os elementos de armazenamento não podem ter mais de uma cópia do arquivo $f$. Todo elemento de processamento conectado a um site acessa réplicas dos arquivos armazenados no próprio site local.

A fim de provar que o problema de acesso a dados é NP-Completo, é necessário demonstrar que ele pertence ao conjunto NP e que ele é NP-Difícil. Para demonstrar que PAD está em NP, uma redução a partir do problema de alocação de múltiplas cópias de um mesmo arquivo em ambientes distribuídos pode ser realizada. Esse problema é denominado Multiple File Allocation (MFA), no qual é NP-Completo de acordo com Garey e Johnson (1990). O problema MFA pode ser definido como:

Instância: Dado o grafo $G(V, E)$, para cada $v \in V$ tem-se uma utilização 
Tabela 4.4: Parâmetros utilizados na definição do problema de acesso a dados.

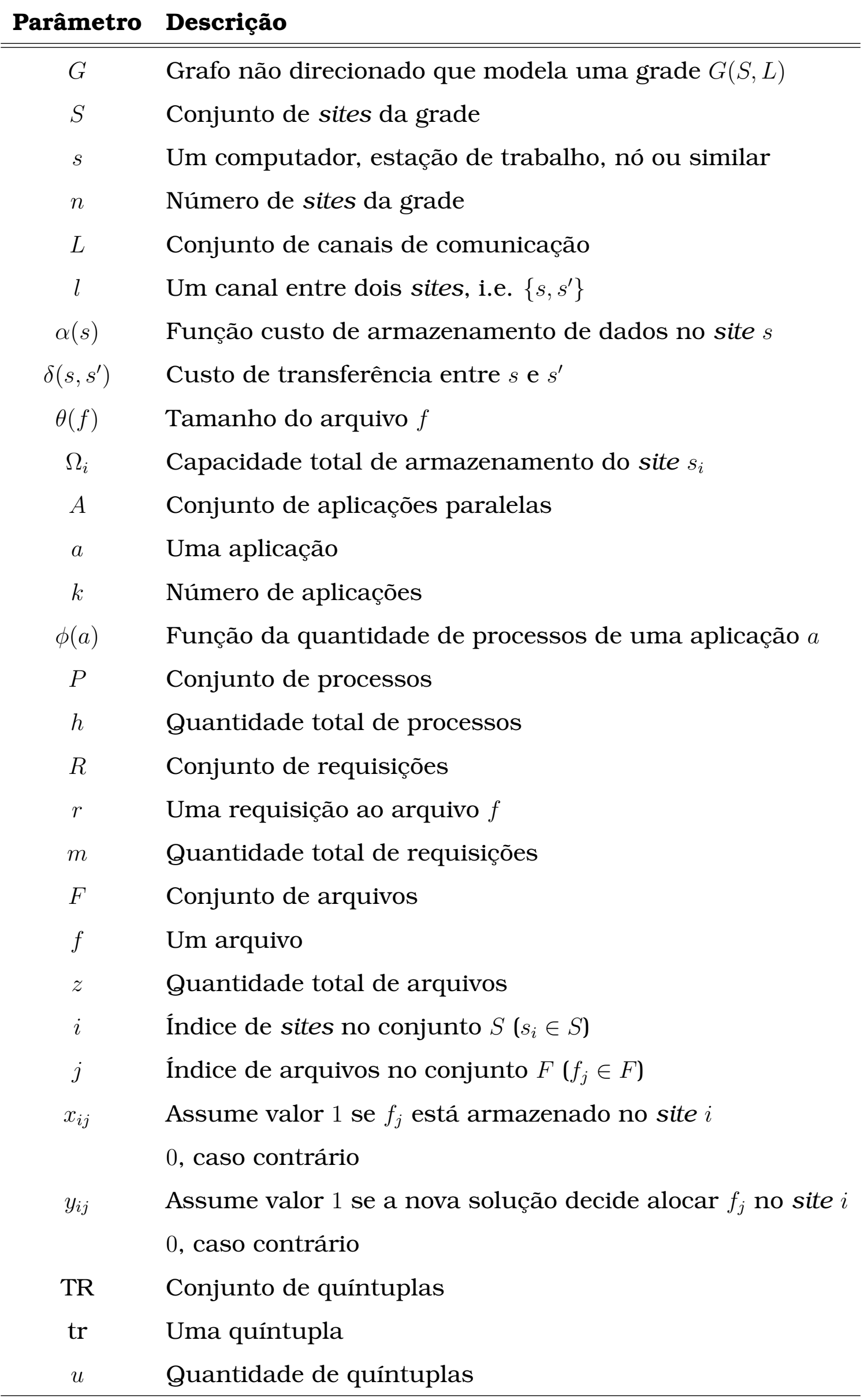


$u(v) \in \mathbb{Z}^{+}$, um custo de armazenamento $s(v) \in \mathbb{Z}^{+}$e um inteiro positivo $K$.

Questão: Há um subconjunto $V^{\prime} \subseteq V$ tal que, se para cada $v \in V$ tem-se $d(v)$ que denota o número de arestas no menor caminho em $G$ a partir de $v$ com destino em um membro de $V^{\prime}$, tal que

$$
\sum_{v \in V^{\prime}} s(v)+\sum_{v \in V^{\prime}} d(v) \times u(v) \leq K ?
$$

Teorema 1. PAD é NP-Completo.

Garey e Johnson (1990) definem três abordagens diferentes para a demonstração de problemas NP-Completos: restrição, substituição local e projeto de componentes. Considere um problema $\Pi \in \mathrm{NP}$, uma prova que utilize a abordagem de restrição consiste em demonstrar que existe em $\Pi$ um caso especial de problema NP-Completo conhecido $\Pi^{\prime}$. A ideia principal está na especificação de restrições adicionais em instâncias de $\Pi$ e, portanto, o problema resultante é semelhante a $\Pi^{\prime}$. O problema $\Pi$ não necessita ser exatamente idêntico a $\Pi^{\prime}$, mas ele deve preservar as respostas correspondentes (sim ou não) em suas saídas.

Na substituição local, deve-se, inicialmente, identificar componentes ou blocos, os quais integram uma instância bem conhecida do problema NP-Completo $\Pi^{\prime}$. A fim de demonstrar que o problema $\Pi$ é NP-Completo, busca-se por similaridades de componentes básicos de $\Pi^{\prime}$, relacionando-os com П. A última abordagem é o projeto de componentes que considera elementos importantes do problema para projetar componentes que são combinados e representam uma instância de um problema NP-Completo bem conhecido.

Demonstração. Para demonstrar que PAD é NP-Difícil foi utilizada a abordagem de restrição. PAD pertence a NP se é possível construir, de forma não determinística, e verificar o grafo $G(S, L)$, em tempo polinomial. Na fase de construção, define-se de maneira não determinística: para cada $s \in S$ uma alocação $x_{i j}$ na qual um arquivo $f_{j}$ é mapeado, e, para cada $l \in L \mathrm{um}$ custo associado $\alpha\left(s, s^{\prime}\right)$. A fase de verificação checa, para cada $f \in F$, se o mapeamento no site é válido, ou seja, se $\sum_{j}^{n} x_{j} \leq n$. Finalmente, a condição $\sum_{j}^{n} y_{i j}=1, \quad i=1, \ldots, n$ é avaliada para a nova alocação dos arquivos em $F$.

Consequentemente, uma instância de PAD é traduzida em uma de MFA. Inicialmente, cada elemento do conjunto $V$ é mapeado em um elemento de $S$, bem como define-se a quantidade de arquivos $z$. Esses arquivos são, inicialmente, alocados nos sites de $S$ de maneira aleatória. Tal alocação deve satisfazer a restrição de que cada site $s \in S$ tem associado capacidade máxima 
de armazenamento $s(v)$. Além disso, $d(v)$ é mapeado em $d\left(s, s^{\prime}\right)$, que representa o custo de transferência de um arquivo $f \in F$ entre dois sites $s$ e $s^{\prime}$. A função $u(v)$ é mapeada em $\theta(f)$, que modela o tamanho dos arquivos. Para cada elemento $e \in E$ existe um correspondente $l \in L$ com largura de banda e latência de rede associadas.

No problema MFA, a Equação 4.8 define a função objetivo que visa minimizar custos relacionados com a alocação de múltiplas cópias de um mesmo arquivo. Essa equação é delimitada pela constante $K$. Similarmente, no problema PAD, a função objetivo é definida na Equação 4.5 que também visa minimizar custos de acesso a dados.

Por fim, considere uma instância de PAD onde: dada uma requisição $r \in$ $R$, realizada por um processo $p \in P$, solicitando uma operação de leitura ou escrita sobre um arquivo $f \in F$. Para qualquer instância de $\mathrm{PAD}$, onde $|S| \geq 2$ e $|L| \geq 2$, o problema apresenta características exponenciais e, portanto, pode ser considerado NP-Completo.

Existem diferentes maneiras de abordar o problema de acesso a dados, dentre elas há técnicas exatas e aproximadas. Abordagens exatas garantem uma solução ótima para o problema. Entretanto, elas podem consumir muito tempo de processamento, dependendo das características do problema e do tamanho de suas instâncias. Para instâncias reduzidas, podem fornecer tempo de execução aceitável, mas, para instâncias maiores, podem ser proibitivas (Voß, 2001). Isso motivou o desenvolvimento de estratégias aproximadas, que consideram heurísticas e meta-heurísticas. Uma heurística é um algoritmo que, baseado no conhecimento ou experiência sobre o problema, leva a soluções adequadas, porém, sem garantias da obtenção de soluções ótimas. As heurísticas são comumente empregadas, pois consideram relações aceitáveis entre a qualidade da solução e a complexidade de tempo. Em contrapartida, as meta-heurísticas são heurísticas generalizadas que resolvem uma classe de problemas e não um problema específico. Exemplos de meta-heurísticas são: Algoritmos Genéticos (AG) (Goldberg, 1989), Colônia de Formigas (ACO - Ant Colony Optimization (Dorigo e Di Caro, 1999)) e Simulated Annealing (SA) (Kirkpatrick et al., 1983).

Nesse sentido, algumas das principais meta-heurísticas foram estudadas e seus conceitos apresentados no Apêndice A. Essas técnicas foram inicialmente investigadas e consideradas para a otimização de acesso a dados, motivadas por resultados relevantes obtidos em trabalhos como de Mello et al. (2007); Souza et al. (2006); Ishii et al. (2007). No entanto, ao avaliar essas abordagens observou-se que as funções objetivo levam tempo considerável para avaliar informações de acessos. Para evitar isso, buscou-se agrupar 
uma janela contígua de acessos e, portanto, otimizar com base em um grupo consecutivo de operações, o que, por outro lado, gerou otimizações em função de comportamentos médios das operações. Esse tipo de otimização não produz bons resultados, uma vez que há variação considerável no comportamento dos processos. Soluções mais eficientes seriam obtidas ao compreender variações comportamentais dos processos e adaptar decisões de otimização de acesso, antecipando operações de leitura e escrita em arquivo.

A fim de propor uma abordagem com essas características, é necessária a identificação da dinâmica comportamental de processos que executam sobre o sistema.

\subsection{Primeira abordagem proposta: utilização de informações históricas}

Dadas as limitações apresentadas por abordagens que empregam meta-heurísticas e algoritmos exatos, esta tese propõe uma nova heurística para otimizar acesso a dados que, inicialmente, utilizou somente informações históricas sobre o comportamento de aplicações. Essa heurística avalia o momento atual de execução dos processos de aplicações, verifica similaridades com o histórico de informações monitoradas e, define uma janela de possiveis eventos futuros (leitura e escrita em arquivos) a serem executados.

O comprimento dessa janela de eventos foi inicialmente definido de maneira fixa. Experimentos foram conduzidos e observou-se o impacto causado pela variação no comprimento da janela (Ishii e de Mello, 2009). Nesse primeiro trabalho, o administrador do sistema ficava responsável por definir o tamanho da janela, o que, comprovadamente, variava muito os resultados. Por outro lado, observou-se que para determinados comprimentos de janela, os resultados de otimização eram bons e, portanto, reduzia o tempo de resposta de aplicações sobre ambientes distribuídos. Isso motivou a proposta de uma abordagem de janela deslizante adaptativa, definida na Subseção 4.4.2. Essa heurística utiliza, portanto, o histórico de informações de comportamento para estimar e, finalmente, antecipar operações de leitura e escrita em arquivos distribuídos.

Os eventos futuros estimados são, em seguida, utilizados pela heurística para tomar decisões de replicação, migração e manutenção de consistência dos dados. Ao migrar ou replicar dados de maneira antecipada, pode-se reduzir custos de acesso, ou seja, dados previamente disponíveis no site local evitam atrasos de acesso.

A abordagem proposta segue a metodologia descrita na Figura 4.2, cujos módulos são: 1) captura e formação da base de conhecimento das aplicações; 
2) janela deslizante adaptativa de eventos futuros; e 3) heurística.

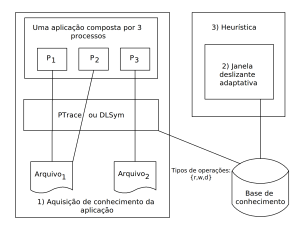

Figura 4.2: Descrição da metodologia proposta para otimização de acesso a dados composta por três módulos: base de conhecimento, janela deslizante e heurística.

\subsubsection{Captura e composição da base de conhecimento}

Há duas abordagens principais para a extração de comportamento de aplicações: monitoramento e interceptação. A primeira solicita, periodicamente, informações de estado para o sistema em estudo. Exemplos de ferramentas de monitoração são o Self-Monitoring Analysis and Reporting Technology (SMART) (SMART, 2010), o Linux Vmstat (Ware e Frederick, 2010) e o Tcpdump (Jacobson et al., 2010). Os sistemas de monitoramento são, geralmente, baseados em contadores, isto é, variáveis que capturam a utilização do sistema dentro de intervalos de tempo específicos. Considere como exemplo o SMART, em um dado instante de tempo foi solicitada a quantidade de leituras em disco e obteve-se 0 como resposta. Após 10 segundos, foi requisitada, novamente, a mesma informação e o sistema retornou 5, o que significa que cinco leituras foram executadas entre o primeiro e o segundo ponto de monitoramento. Essa abordagem captura a quantidade de dados e qual tipo de evento ocorreu no intervalo de tempo, mas não informa "quando" esses eventos ocorreram.

A segunda abordagem intercepta eventos de processos e chamadas de procedimento, capturando informações relevantes tais como o momento exato de ocorrência do evento, id do processo, quantidade de bytes lidos ou escritos, id do arquivo acessado etc. Entretanto, dependendo da complexidade do evento (custos envolvidos), a interceptação pode tornar-se proibitiva. Exemplos de ferramentas de interceptação são Unix DLSym (Jung et al., 2007) e Ptrace (Spillane et al., 2007).

DLSym permite a interceptação de chamadas a procedimentos dinâmicos (a partir de bibliotecas dinâmicas). Assim que um programa invoca uma função, ao invés de executar o código interno dessa função, carrega-se uma biblioteca compartilhada. Isso evita o desenvolvimento de um novo procedimento e também permite a interceptação de chamadas. Consequentemente, qualquer procedimento em uma biblioteca dinâmica compartilhada pode ser interceptado, o que auxilia e suporta a construção de ferramentas de monitoramento. 
A Ptrace intercepta, transparentemente, sinais de processos e chamadas de sistema. Ela é, usualmente, empregada no desenvolvimento de ferramentas de diagnóstico e depuração. Essa ferramenta não utiliza bibliotecas dinâmicas e pode interceptar quaisquer aplicativos baseados em Unix (Spillane et al., 2007).

A fim de escolher a melhor abordagem para um dado sistema, pesquisadores devem avaliar alternativas entre monitoramento e interceptação. Certos sistemas podem somente ser monitorados, enquanto outros, somente interceptados. Dadas suas características físicas, hardware são geralmente monitorados, enquanto software podem ser monitorados ou interceptados. Nesta tese, a abordagem de interceptação foi escolhida, pois permite a extração de comportamento de aplicações de maneira mais precisa (quando o evento ocorre no tempo). Além disso, o monitoramento pode ocultar detalhes de comportamento entre intervalos de coleta.

Após a extração do comportamento de aplicações é necessário transformar a sequência de eventos obtidos em séries com valores numéricos que representem instantes de tempo. Nesta tese, todo evento é representado por uma quíntupla $\mathrm{tr}=\{\mathrm{pid}$, inode, qtd, time, op $\}$ onde, pid é a identificação do processo que realizou uma operação sobre os dados, inode é a identificação do arquivo, qt d é a volume de dados lidos ou escritos, time é o intervalo entre operações e op é o tipo de operação, leitura (r), escrita (w) ou ausência de operação ( $\mathbf{d}$, i.e. estado idle).

\subsubsection{Janela deslizante adaptativa}

O comprimento da janela deslizante define a quantidade de eventos futuros que a heurística irá analisar a fim de tomar decisões de otimização, impactando, significativamente, no desempenho global do sistema. O impacto que a janela deslizante causa no desempenho de uma aplicação é apresentado na Figura 4.3, que considera uma janela deslizante de comprimento fixo. No gráfico da Figura 4.3, quatro técnicas são avaliadas LRU, LFU, Modelo econômico (ver Subseção 4.4.4), bem como uma heurística que considera comprimento fixo da janela deslizante (identificada pela sigla HEU na Figura 4.3) (Ishii e de Mello, 2009).

É possível observar que, na janela de comprimento 4 o tempo de execução da aplicação é maior. Isso ocorre, principalmente, devido à heterogeneidade e à interposição das operações de leitura e escrita. Esse fato também pode ser observado nas janelas de comprimento 200 e 500, demonstrando elevado grau de heterogeneidade no comportamento da aplicação estudada. Os experimentos permitiram observar que, quanto mais homogênea é a ordem de execução das operações de leitura e escrita (i.e., operações do 
Tabela 4.5: Exemplo de um arquivo de traços de execução, onde cada linha representa uma operação do processo $p_{i}$ sobre um arquivo identificado por $f_{j}$.

\begin{tabular}{cccccc}
\hline & pid & inode & qtd & time & op \\
\hline \hline 1 & $p_{41}$ & $f_{0}$ & 313 & 24 & $\mathbf{r}$ \\
2 & $p_{89}$ & $f_{3}$ & 94 & 171 & $\mathbf{w}$ \\
3 & $p_{10}$ & $f_{9}$ & 92 & 80 & $\mathbf{w}$ \\
4 & $p_{32}$ & $f_{0}$ & 826 & 132 & $\mathbf{w}$ \\
5 & $p_{69}$ & - & - & 76 & $\mathbf{d}$ \\
6 & $p_{1}$ & $f_{5}$ & 292 & 70 & $\mathbf{w}$ \\
7 & $p_{39}$ & $f_{7}$ & 26 & 32 & $\mathbf{r}$ \\
8 & $p_{100}$ & $f_{2}$ & 6 & 32 & $\mathbf{r}$ \\
9 & $p_{4}$ & $f_{6}$ & 126 & 32 & $\mathbf{w}$ \\
10 & $p_{13}$ & $f_{5}$ & 5.286 & 32 & $\mathbf{r}$ \\
11 & $p_{22}$ & $f_{2}$ & 64 & 32 & $\mathbf{w}$ \\
\hline
\end{tabular}

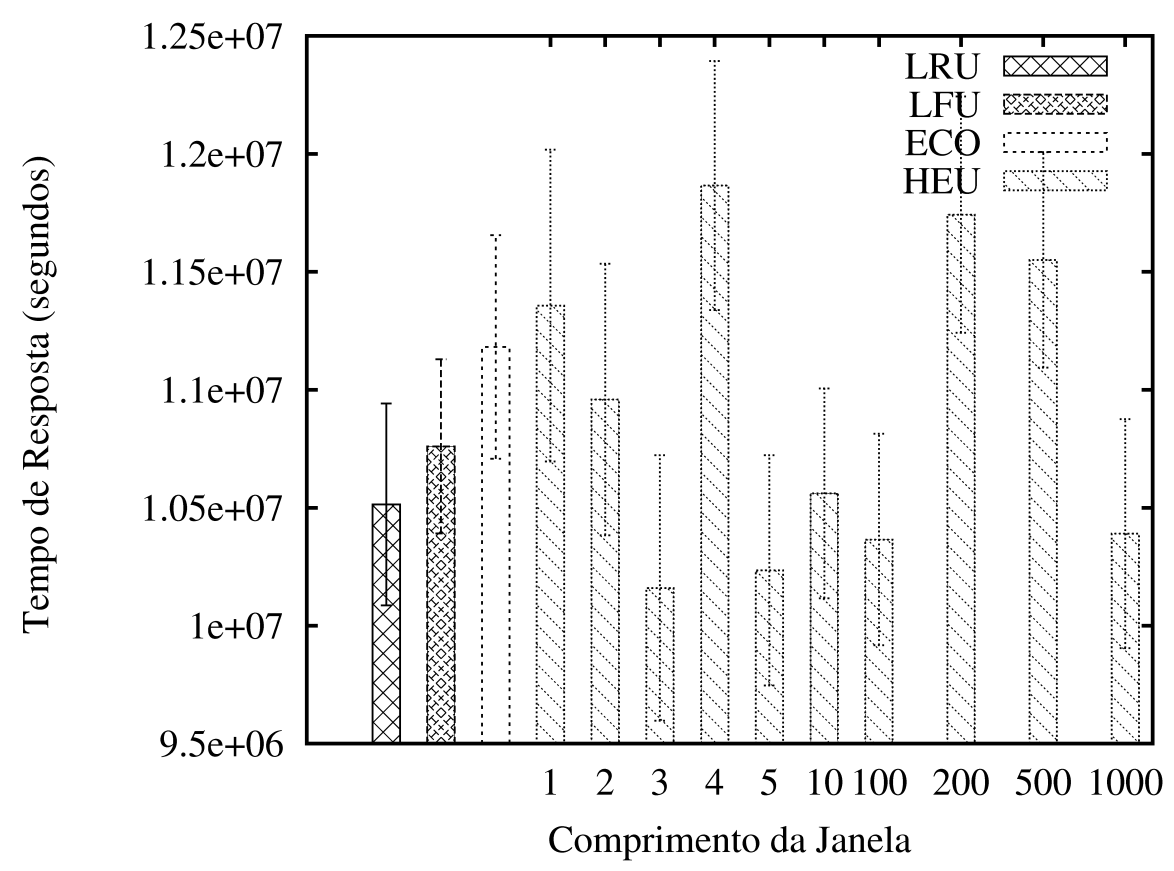

Figura 4.3: Gráfico com resultados de experimentos comparativos entre estratégias LRU, LFU, ECO e HEU com janela de comprimento fixo. É interessante observar os impactos proporcionados com a variação no comprimento da janela. 
mesmo tipo estão organizadas consecutivamente), pode-se definir um maior horizonte de estimação, ou seja, mais eventos futuros podem ser estimados e, consequentemente, o comprimento da janela pode ser ajustado para um valor maior.

Esse fato motivou a proposta de uma abordagem com janela deslizante adaptativa que considera a quantidade e o tipo das operações. Assim, foi proposto o parâmetro $\beta$ que é utilizado para adaptar o comprimento da janela, de acordo com a quantidade de operações homogêneas e consecutivas (i.e, sequências de leituras ou de escritas). A Equação 4.9 define a Janela Deslizante Adaptativa (JDA) proposta, onde $J_{t+1}$ é o comprimento da próxima janela, $J_{t}$ o comprimento da janela atual, Op é a quantidade de operações homogêneas e $\beta$ é o parâmetro que determina a característica da atualização (se mais agressiva ou moderada) no comprimento da janela.

$$
J_{t+1}=J_{t} \times(1-\beta)+\mathrm{Op}^{2 \times \frac{\mathrm{Op}}{J_{t}}} \times \beta
$$

Considere como exemplo $J_{1}=10$, Op $=4$ e $\beta=0,10$, o qual resulta em uma próxima janela dada por $J_{2}=9$. Note que o comprimento da janela adapta-se de acordo com a quantidade de operações do mesmo tipo, tal como apresentado na Figura 4.4. Em outras palavras, se Op é igual a $J_{t}$ (assim como a janela $J_{4}$ da Figura 4.4) o comprimento da próxima janela aumenta consideravelmente, ou seja, $J_{5}=11$. O parâmetro $\beta$ foi definido experimentalmente e uma discussão mais elaborada é apresentada na Subseção 4.4.8.

A Figura 4.4 apresenta um exemplo de arquivo de traços com informações de comportamento de processos (nesse exemplo as informações estão restritas às operações de leitura e de escrita) obtidas através de interceptação (Seção 4.4). A primeira parte da Figura 4.4 mostra o comprimento inicial da janela deslizante $J_{1}=10$ e todas as operações (ou eventos) subsequentes. A segunda parte demonstra os passos para o cálculo da próxima janela baseado na Equação 4.9. Finalmente, a última parte dessa figura apresenta todas as janelas obtidas a partir do arquivo de traços original, ou seja, $J_{2}=9, J_{3}=$ $8, J_{4}=7$ e $J_{5}=11$. Esses resultados demonstram que os comprimentos de janela adaptam-se de acordo com o comportamento das operações de leitura e escrita.

Por meio desse mecanismo, é possível calcular a quantidade de operações similares e o comprimento da próxima janela de eventos, limitando o número de eventos analisados no futuro (na próxima janela). Esses eventos são considerados pela heurística proposta para transferir, antecipadamente e em meio de armazenamento local, dados que serão utilizados por processos. Essas operações reduzem custos de acessos futuros. 


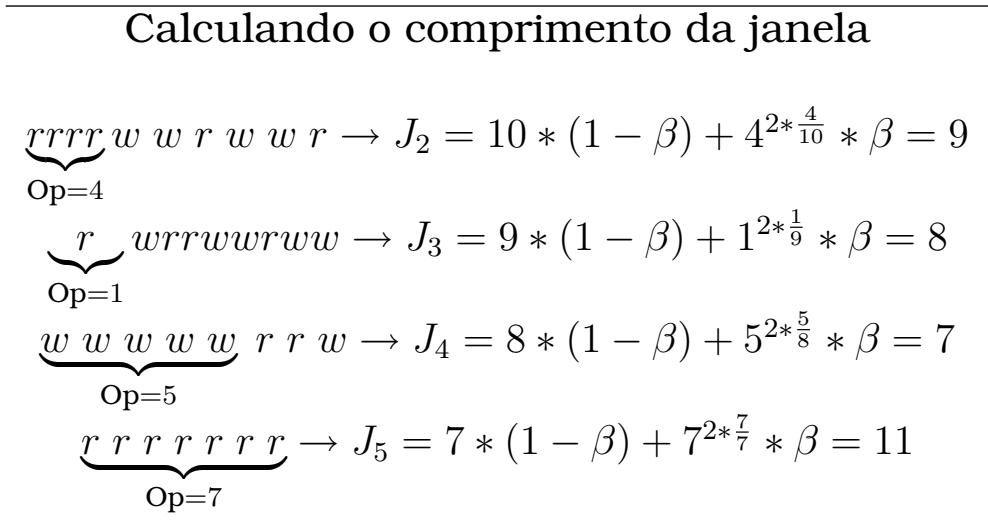

Comprimento das janelas obtido após execução da JDA

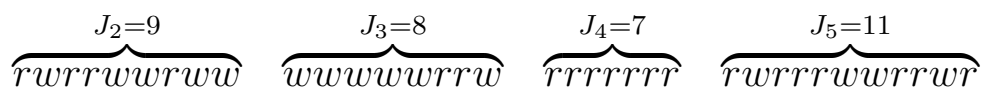

Figura 4.4: Exemplo de funcionamento da abordagem JDA com $\beta=0,10$.

Sem o mecanismo de adaptação é necessário atribuir manualmente o comprimento da janela deslizante (Ishii e de Mello, 2009). Isso requer a avaliação experimental de diferentes valores para escolha do comprimento mais adequado. Além disso, um comprimento de janela fixo pode ser adequado para uma aplicação específica, enquanto outras podem apresentar queda de desempenho. Portanto, o comprimento é um atributo que requer adaptação dinâmica de acordo com o comportamento instantâneo dos processos que compõem determinada aplicação. Dessa maneira, motiva-se o uso do mecanismo adaptativo no qual o comprimento da janela acompanha o comportamento de cada processo no tempo, deixando o mecanismo mais eficiente e flexível às mudanças no padrão de acesso aos dados.

\subsubsection{Heurística proposta}

Considere o conjunto $P=\left\{p_{0}, p_{1}, \ldots, p_{h-1}\right\}$ que contém todos os processos previamente escalonados nos sites da grade, o conjunto $F=\left\{f_{0}, f_{1}, \ldots, f_{z-1}\right\}$ de arquivos acessados, o conjunto de operações $O=\{r, w, d\}$, o conjunto de eventos TR que modela séries temporais de comportamento dos processos, onde cada elemento $\operatorname{tr} \in$ TR descreve uma operação op sobre um arquivo $f$, executado pelo processo $p$.

Assim, o objetivo é antecipar operações de acesso a dados sobre TR por meio de uma janela deslizante adaptativa JDA $\rightarrow\left[t_{y}, t_{x}\right]$ (considerando $x>y$ ). 
Essa janela define a quantidade de eventos futuros estimados (comportamento da aplicação) que auxilia na otimização de operações de leitura e escrita.

O mecanismo de antecipação visa a transferência de arquivos antes que sejam solicitados por processos em execução. Isso reduz custos de acesso, pois o arquivo é requisitado e replicado, localmente, antes de sua utilização, reduzindo o tempo de execução das aplicações. Na Figura 4.5, um arquivo é requisitado no instante $t_{2} \mathrm{e}$, neste caso, totalmente transferido antes de seu uso. Em outro exemplo, Figura 4.6, o arquivo é solicitado no instante $t_{2}$, entretanto, a transferência não é completamente finalizada e, neste caso, a heurística ainda necessita de tempo hábil para transferir os dados remanescentes. Nessa situação, o custo total de acesso é dado por $t_{3}-t_{2}$.

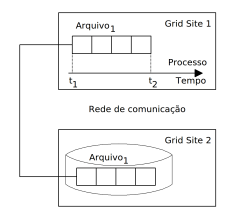

Figura 4.5: Exemplo de transferência completa de um arquivo.

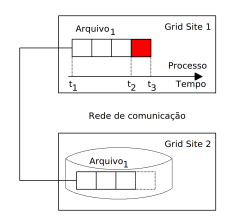

Figura 4.6: Exemplo de transferência parcial de um arquivo.

Tal como apresentado previamente na Tabela 4.5, cada evento corresponde a uma quíntupla $\operatorname{tr} \in \mathrm{TR}$. Assim, a heurística proposta nesta tese (Algoritmo 5) considera o histórico de comportamento de aplicações, representado pelo conjunto TR e uma JDA de eventos futuros a fim de otimizar operações de acesso a dados em ambientes distribuídos. A antecipação de operações futuras suporta a minimização de custos de comunicação/sincronismo relacionados à transferência de arquivos e consistência.

Dessa maneira, considere um processo $p$ que realiza operações sobre um arquivo $f$. Agora, assuma que $p$ está no instante de tempo atual ou $t_{c}$ e há uma janela de eventos que termina no instante $t_{f}$. A heurística avalia se $o$ arquivo $f$ pode ser recuperado (i.e. localiza a última versão do arquivo ou réplica $f^{\prime}$ ) para o site local, onde ele foi requisitado. Se isso ocorrer, os custos de transferência e atrasos de acesso são reduzidos a valores próximos de 0 , bem como o desempenho da aplicação aumenta significativamente. Nessas circunstâncias, existem dois modos de recuperação de $f$, ambos implicam no custo $\Gamma$ (Equação 4.5): 
1. se $t_{c}+\Gamma \leq t_{f}$, então o arquivo $f$ pode ser totalmente recuperado, o que reduz o tempo de execução do processo em $\Gamma$ unidades de tempo;

2. se $t_{c}+\Gamma>t_{f}$, a redução é de $t_{f}-t_{c}$ unidades de tempo.

A heurística proposta é definida no Algoritmo 5 e outros procedimentos auxiliares são descritos nos Algoritmos 1, 2, 3 e 4.

O procedimento Recupera(f) (Algoritmo 1) estabelece uma nova cópia local (por meio de replicação) do arquivo remoto $f$ e avalia a possibilidade de remoção (com propósitos de migração dos dados). O procedimento Leitura(tr) (Algoritmo 2) recebe como entrada uma quíntupla tr e, caso $f$ esteja inválido, então, aplica-se o método Recupera $(f)$ e, em seguida, realiza-se a leitura. O procedimento Escrita(tr) tem o mesmo funcionamento da leitura (Algoritmo 3), com a exceção de propagar alterações para as demais réplicas do arquivo modificado. O procedimento Invalida $(f)$ recebe como entrada um arquivo $f$ e atualiza o estado de todas as suas réplicas como inválido (Algoritmo 4).
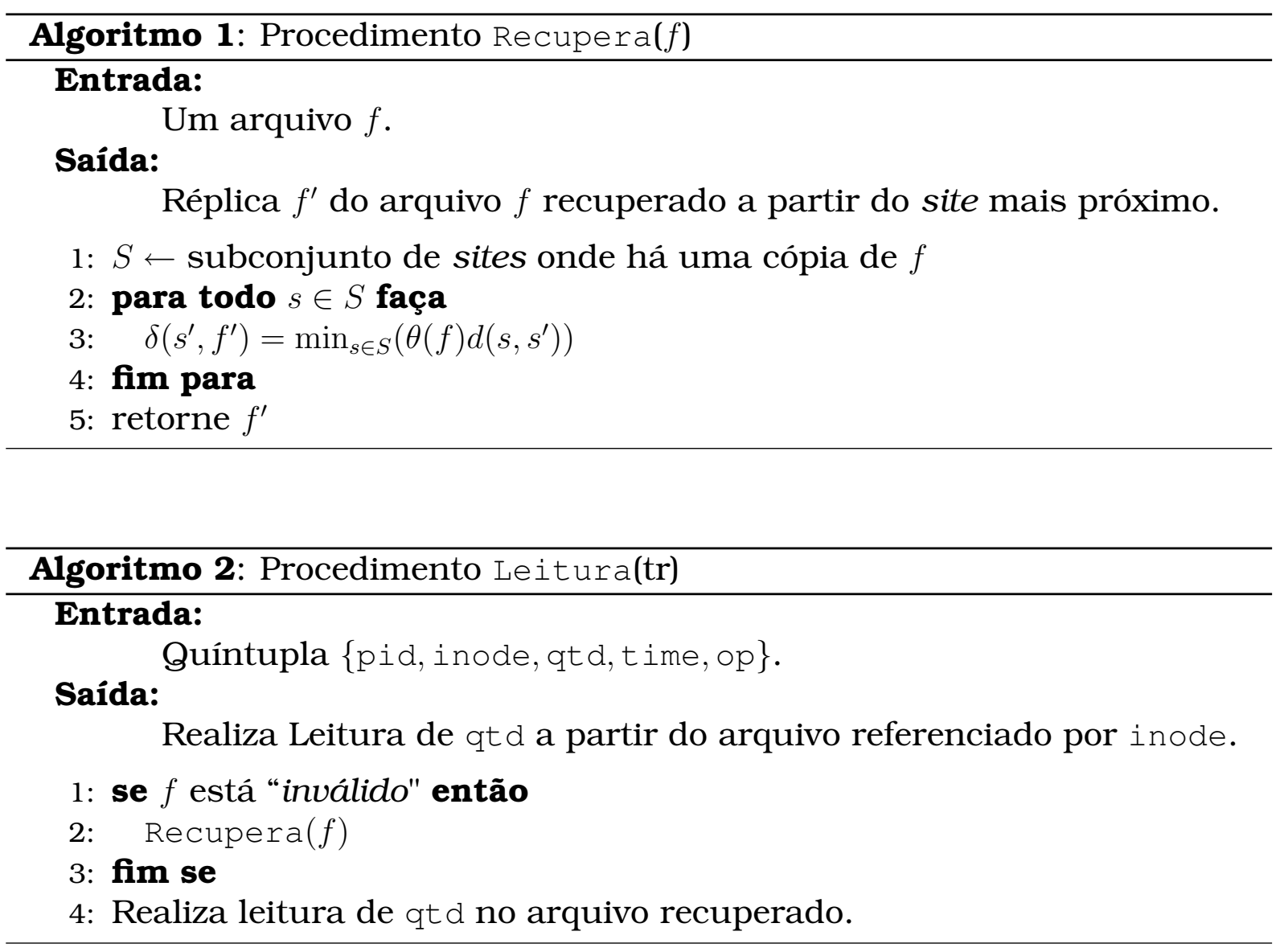

Cada site $s \in S$ (onde o ambiente é representado por um grafo $G(S, L)$ ) tem associado um conjunto de processos que foram previamente escalonados no ambiente, considerando qualquer política. O procedimento getNextProcess $(s)$, Algoritmo 5 linha 3, devolve um processo $p$ que foi escalonado no site $s$. Todo site $s$ contém um histórico com traços de execução 

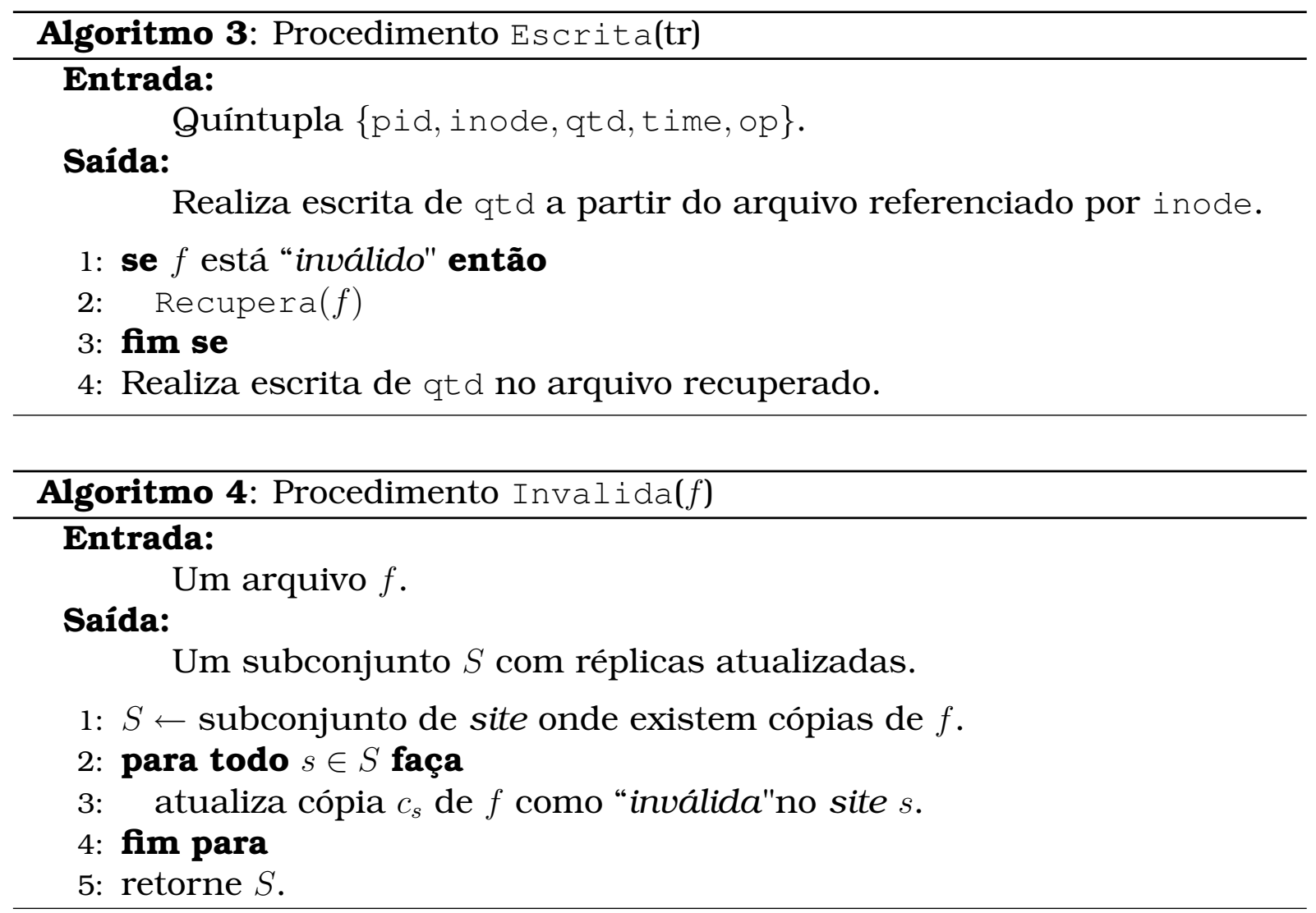

dos processos (TR, linha 4). O pseudocódigo entre as linhas 5 e 13 realiza operações tr $\in$ TR, considerando os tipos: leitura, escrita ou inativo. Da linha 14 até a 39, a heurística avalia a viabilidade de replicação, migração, consistência e recuperação de arquivos, de acordo com o histórico de eventos dentro da janela deslizante. As linhas 31 e 32 calculam a quantidade de operações similares dentro da janela atual.

A linha 41 corresponde à situação em que o site $s$ não é responsável pelo processo $p$ e não existe eventos na janela dentro desse intervalo de execução, consequentemente, $s$ permanece inativo por um período de time unidades de tempo.

\subsubsection{Experimentos}

Em pesquisas da área de computação de alto desempenho e distribuída existem, basicamente, duas metodologias para condução de experimentos: reais ou simulados (Dang e Lim, 2007). Experimentos em ambientes reais são geralmente precisos e confirmam na prática a abordagem proposta. Entretanto, aplicações podem executar por longos períodos de tempo e, além disso, necessitam ser totalmente e corretamente implementadas. Nesses ambientes, a maioria das falhas ocorrem durante a execução, o que influencia na precisão dos resultados. Além disso, outras cargas de trabalho submetidas ao sistema podem interferir nos resultados experimentais. 


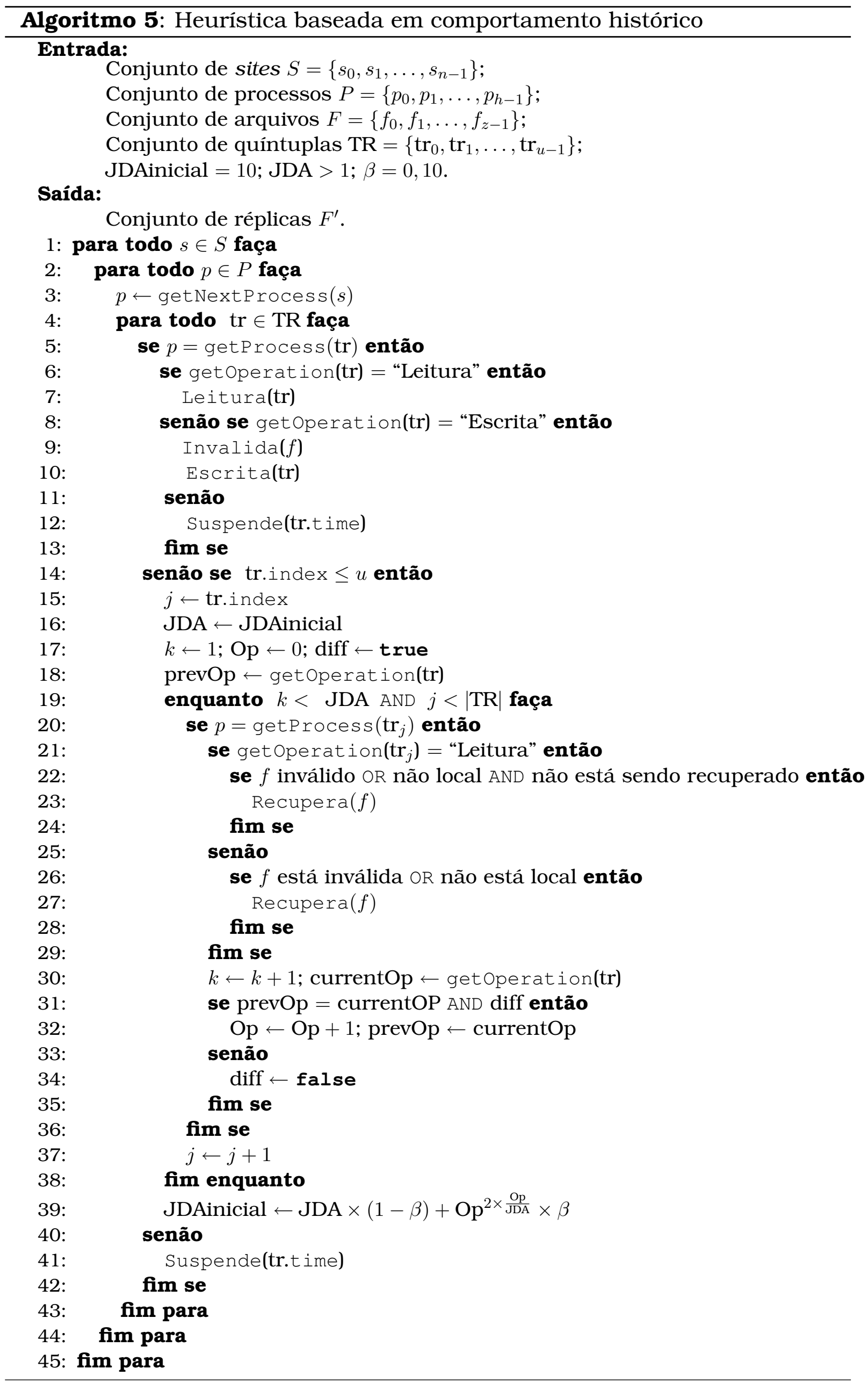


Finalmente, torna-se muito difícil ter um ambiente totalmente controlado e dedicado que evite tais interferências, principalmente, considerando ambientes inerentemente de larga escala.

Simulações aproximam experimentos reais por meio da modelagem (uso de equações) do ambiente em estudo e de suas interações (entre computadores, processos, operações, etc.). As vantagens da simulação sob experimentos reais são: não há necessidade do desenvolvimento completo do sistema real; não há limites para os estudos de caso (cenários) experimentados; controle total do ambiente e de seus parâmetros e, por fim, a possibilidade de reprodução total dos experimentos.

As vantagens da simulação motivaram, no contexto desta tese, a adoção do simulador OptorSim, vinculado ao projeto European DataGrid (EDG) (Bell et al., 2003). Esse simulador foi originalmente desenvolvido com propósitos da modelagem dos efeitos dinâmicos causados pela replicação de dados. Ele é utilizado em diversas áreas e aplicações tal como Large Hadron Collide Computing Grid (LCG), e também empregado em outros trabalhos relacionados (Kim et al., 2009; AL-Mistarihi e Yong, 2009).

O OptorSim modela ambientes distribuídos, sendo principalmente indicado para representar grades computacionais, por meio de sites interconectados através de canais de comunicação, tal como apresentado na Figura 4.7. Cada site contém ao menos um elemento de processamento ( $\mathrm{EP}$, ou computador), um elemento de armazenamento (EA), ou ambos. Cada site executa um otimizador de réplicas (OR), que toma decisões de replicação, e contém um gerenciador de processos (GP), que decide sobre o escalonamento de processos. O simulador modela processos acessando um certo subconjunto de arquivos, que podem ser replicados entre os sites de acordo com o OR. Há um catálogo de réplicas (CR) que mapeia nomes lógicos para arquivos físicos e um gerenciador de réplicas (GR), que trata replicações e também faz registros e remoções no $\mathrm{CR}$, mantendo-o sincronizado. A Figura 4.7 apresenta todos os componentes do simulador OptorSim.

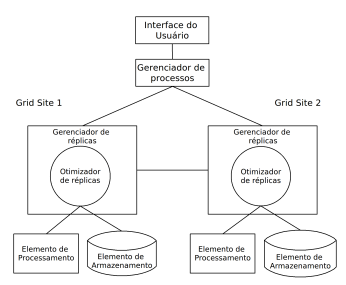

Figura 4.7: Arquitetura do simulador OptorSim para grades computacionais que suporta modelos de replicação de dados.

Esse simulador permite que usuários especifiquem a topologia do ambiente distribuído por meio da parametrização dos EP's e dos canais de comunicação. Nesta tese, emprega-se um gerador de topologia denominado BRITE (Medina 
et al., 2001), baseado em conceitos de redes complexas (Albert e Barabasi, 2001), o qual fornece informações de largura de banda e latência para canais de comunicação. Geradores de topologia são frequentemente utilizados na modelagem de ambientes de larga escala (Medina et al., 2001), tal como a Internet. O BRITE gera arquivos de saída contendo canais de comunicação com largura de banda e latências associadas, que são utilizados como entrada para o OptorSim.

O OptorSim ainda requer a definição de uma lista de processos associados a um conjunto de arquivos. Esses processos podem acessar um subconjunto de arquivos de acordo com seu padrão de acesso. O simulador também implementa quatro algoritmos de escalonamento: Random, QueueLength, Accesscost e QueueAccesscost. O algoritmo Random atribui aleatoriamente um processo a um EP, muito similar ao escalonamento do software Legion (Chapin et al., 1999). O QueueLength escalona as tarefas nos EP's com menor comprimento da fila de processos, a fim de evitar a ociosidade de recursos e prover balanceamento de carga. O Accesscost estima o tempo de acesso de todos os arquivos requisitados por um processo e, então, faz o escalonamento no EP com menor custo estimado. O último algoritmo é similar ao anterior, exceto por considerar o comprimento médio da fila de processos, ou seja, uma abordagem híbrida.

Além dos algoritmos de escalonamento, o OptorSim fornece cinco técnicas de otimização de acesso a arquivos: 1) Na técnica Simpleoptimiser, nenhuma replicação é realizada, isto é, os arquivos são remotamente acessados; 2) DeleteoldestFileoptimiser replica arquivos no instante em que os processos solicitam, removendo a réplica menos recentemente utilizada (LRU); 3) DeleteLeastAccessedFileoptimiser replica arquivos no instante em que os processos solicitam, removendo a réplica menos frequentemente utilizada (LFU); 4) EcoModeloptimiserBinomial considera um modelo econômico para determinar as replicações. Nessa técnica, as réplicas são removidas de acordo com uma função Binomial de estimativa de acesso; 5) EcoModeloptimiserzipf considera um modelo econômico para a replicação de arquivos. As réplicas são removidas de acordo com uma função de estimativa Zipf (Ferrer-i-Cancho e Sole, 2002). As técnicas LRU, LFU e EcoModeloptimiserzipf foram utilizadas em experimentos (Subseção 4.4.8), a fim de compará-las com a heurística proposta na Subseção 4.4.3.

O simulador também fornece métricas de desempenho para suas entidades. Para os sites, o OptorSim fornece: quantidade de leituras locais e remotas, percentual de tempo em que os EP's do site permaneceram ativos; número de transferências de arquivos encaminhadas através do site; e tempo médio de resposta (em segundos) de todos os processos submetidos ao site. 
Para os dispositivos de armazenamento, o OptorSim fornece: capacidade e utilização, em MB. O OptorSim também fornece as seguintes métricas para os EP's: tempo de resposta de um processo, em milissegundos; tempo de execução acrescido do tempo em fila; quantidade de leituras locais e remotas; número de processos completos; percentual de tempo em que o EP permaneceu ativo; lista com os arquivos acessados pelo processo no EP; e, finalmente, o tempo total de execução (em segundos) de todos os processos.

\subsubsection{Extensões do OptorSim}

Algumas extensões foram necessárias com objetivo de adaptar o OptorSim às necessidades desta tese. Tais extensões fornecem suporte para: 1) arquivos com traços de execução (log); 2) operações de escrita; e 3) janela deslizante adaptativa de operações registradas em histórico.

O OptorSim provê diferentes padrões de acesso a arquivos: 1) O padrão sequentialAccessGenerator define que os arquivos são sequencialmente acessados; 2) No RandomAccessGenerator, os arquivos são acessados por meio de uma distribuição uniforme; 3) No RandomWalkUnitaryAccessGenerator, uma distribuição caminhada aleatória unitária é utilizada para modelar o acesso aos arquivos; 4) No RandomWalkGaussianAccessGenerator, arquivos são acessados por meio de uma distribuição caminhada aleatória gaussiana; e 5) No RandomZipfAccessGenerator, uma distribuição aleatória Zipf é utilizada para modelar o acesso aos arquivos. Esses padrões de acesso geram, dinamicamente, o comportamento dos processos, o que não satisfaz uma abordagem que emprega histórico de operações. Esse fato, motivou o desenvolvimento de um novo padrão de acesso que foi incorporado ao OptorSim: 6) padrão de acesso aos arquivos baseado em traços de execução, onde cada evento (uma linha no arquivo de traços) representa uma operação de um processo (comportamento). Esses eventos fornecem as mesmas informações descritas na Tabela 4.5 para cada operação.

O simulador, originalmente, fornece somente operações de leitura, assim, foi necessário, também, estendê-lo a fim de incorporar operações de escrita. Essa extensão considera o custo de escrita de dados, Equação 4.3, bem como a propagação de atualizações de réplicas, tal como definido nos Algoritmos 3 e 4 .

Finalmente, foi desenvolvida uma janela deslizante adaptativa de eventos futuros (operações), baseada no histórico de informações, tal como apresentado previamente na Equação 4.9. Experimentos confirmam que essa janela tem forte impacto no desempenho da heurística proposta (Subseção 4.4.8). 


\subsubsection{Parametrização dos experimentos}

No contexto desta tese, foram projetados experimentos sobre grade computacional contendo 128 sites (regiões). Cada site foi configurado com um elemento de armazenamento (EA), cuja capacidade é representada por uma função de distribuição de probabilidade exponencial com média 100GB (parâmetro $\Omega$ descrito na Seção 4.3). Os canais de comunicação foram modelados por meio do gerador de topologias BRITE, seguindo o modelo Barabasi para sistemas autônomos (Medina et al., 2001). Além disso, adotou-se, no BRITE, uma função de distribuição de cauda pesada para modelar a adição de novos EP's e largura de banda, neste caso, a largura de banda mínima definida foi de $10 \mathrm{Mbits} / \mathrm{s}$ e a máxima de $1.024 \mathrm{Mbits} / \mathrm{s}$. Uma função de distribuição de probabilidade exponencial também foi adotada para modelar os atrasos da rede de comunicação com média igual a 0,5 segundos.

Esses experimentos foram configurados para lançar 128 processos um sobre cada site da grade. Mais processos poderiam ser adotados, no entanto, ao considerar conjuntos de processos executando em um site específico, o comportamento de cada processo pode ser visto como parte integrante do comportamento de todos aqueles alocados no mesmo site. Assim, pode-se assumir que o comportamento de um único processo como o comportamento de um conjunto de processos alocados no mesmo site.

Uma função de distribuição de probabilidade uniforme (com média 10) foi adotada para determinar a quantidade de arquivos que um processo acessa durante sua execução. Cada arquivo tem tamanho de 1 GByte. O comportamento dos processos é atribuído ao simulador por meio de um arquivo de configuração, que pode ser sintético ou obtido através de mecanismos de interceptação, tal como discutido na Subseção 4.4.1 e apresentado na Tabela 4.5. Todos os processos tem a mesma probabilidade de execução em cada EP da grade. Uma função de distribuição de probabilidade exponencial foi adotada a fim de modelar o intervalo entre chegadas de processos, cuja média é igual a 1.500 milissegundos.

A capacidade de processamento de um EP é obtida por meio do benchmark SPEC CINT 2000, o qual considera uma metodologia bem aceita para mensurar desempenho de CPUs. Ele gera medidas de desempenho baseadas em uma máquina de referência (a versão adotada considera uma máquina Sun Ultra 10 como base) (Corporation, 2010). Considere como exemplo um processador Pentium IV que tem desempenho 0,5 CINT 2000. Nesta tese, todo EP é homogêneo e tem capacidade de processamento equivalente a $1,0 \mathrm{~K}$ CINT 2000, ou seja, 1.000 CINT 2000. O ambiente foi definido de maneira homogênea, pois isso não influencia as operações de acesso a dados, as quais são objetivo principal desta tese. 
Como o foco desta tese não trata da alocação de processos, foi considerado o mesmo algoritmo de escalonamento para todos experimentos, ou seja QueueAccesscost, descrito na Subseção 4.4.4. Além disso, foi adotada a mesma técnica para modelar o padrão de acesso a dados, ou seja, todas as abordagem utilizam o mesmo arquivo de traços de execução para cada cenário avaliado.

\subsubsection{Avaliação de intrusão na captura de informações}

Toda técnica de interceptação causa algum impacto sobre a execução normal dos processos. Com o objetivo de investigar esse impacto, foram avaliados os custos envolvidos na captura de informações (ou conhecimento) das aplicações, a qual gera arquivos de traços de execução que, posteriormente, são utilizados para a tomada de decisões da heurística proposta (Subseção 4.4.3). Duas técnicas de captura de informações foram avaliadas: Ptrace (Spillane et al., 2007) e DLSym (Jung et al., 2007), utilizando dois benchmarks: Nbench (Mayer, 2010) e Bonnie (Bray, 2009). O Nbench testa a capacidade de CPU e de acesso ao subsistema de memória por meio de operações de ponto flutuante. O Bonnie realiza testes por meio de operações de leitura e escrita em arquivos.

Cada experimento foi executado 30 vezes em um processador Intel Core i $7 \mathrm{CPU} 2.67 \mathrm{GHz}$, 8GB RAM e 250GB HD, obtendo-se médias e desvios padrão. Os cenários foram configurados com o intuito de avaliar o tempo de execução dos benchmarks com os mecanismos de captura habilitados e desabilitados. A Tabela 4.6 apresenta os resultados da intrusão causada pelos mecanismos. No caso da Ptrace o impacto foi inferior a $12 \%$. Para o DLSym, o impacto foi de 1,5\% do tempo total de execução. Cada valor da Tabela 4.6 corresponde ao tempo médio de execução do benchmark e o desvio padrão, respectivamente. Os resultados confirmam que o DLSym é mais adequado para a captura de informações em ambientes reais.

Tabela 4.6: Resultados para captura de informações de processos através de dois mecanismos: Ptrace e DLSym, considerando dois benchmarks: Nbench e Bonnie

\begin{tabular}{cccc}
\hline Benchmark & $\begin{array}{c}\text { Tempo(s) } \\
\text { Desabilitado }\end{array}$ & $\begin{array}{c}\text { Ptrace } \\
\text { Habilitado }\end{array}$ & $\begin{array}{c}\text { DLSym } \\
\text { Habilitado }\end{array}$ \\
\hline \hline Nbench & $272,81 \pm 5,99$ & $309,69 \pm 20,26$ & $276,78 \pm 5,46$ \\
Bonnie & $10,41 \pm 0,03$ & $11,97 \pm 0,07$ & $10,47 \pm 0,04$ \\
\hline Impacto Nbench & - & $11,91 \%$ & $\mathbf{1 , 4 3 \%}$ \\
Impacto Bonnie & - & $13,03 \%$ & $\mathbf{0 , 5 7 \%}$ \\
\hline
\end{tabular}




\subsubsection{Resultados da primeira abordagem}

Nesta seção são apresentados os resultados dos experimentos conduzidos de acordo com o ambiente descrito previamente, ou seja, uma grade com 128 sites. Cada gráfico apresenta os resultados (em barras) que correspondem à média de 30 execuções (esse número é baseado no Teorema do Limite Central (Schefler, 1988), que suporta a obtenção de medidas estatística de resumo significativas).

Os experimentos foram categorizados de acordo com a característica do arquivo de traços, ou seja, o percentual de operações de leitura e escrita. Como exemplo, considere a Figura 4.9 cujo arquivo de traços têm $5 \%$ de operações de escrita, isto é, $5 \%$ dos eventos tem o tipo da operação definido com valor w (ver Tabela 4.5).

Foram avaliadas três técnicas de otimização de acesso a dados: LRU, LFU e Modelo Econômico (ECO), descritas na Subseção 4.4.4 e disponíveis no simulador OptorSim. Assim, o principal objetivo foi comparar os resultados obtidos por essas técnicas com a heurística proposta (HEU) no Algoritmo 5. A heurística HEU foi avaliada sob diferentes valores do parâmetro $\beta$ : $\{0,05 ; 0,10 ; 0,15\}$.

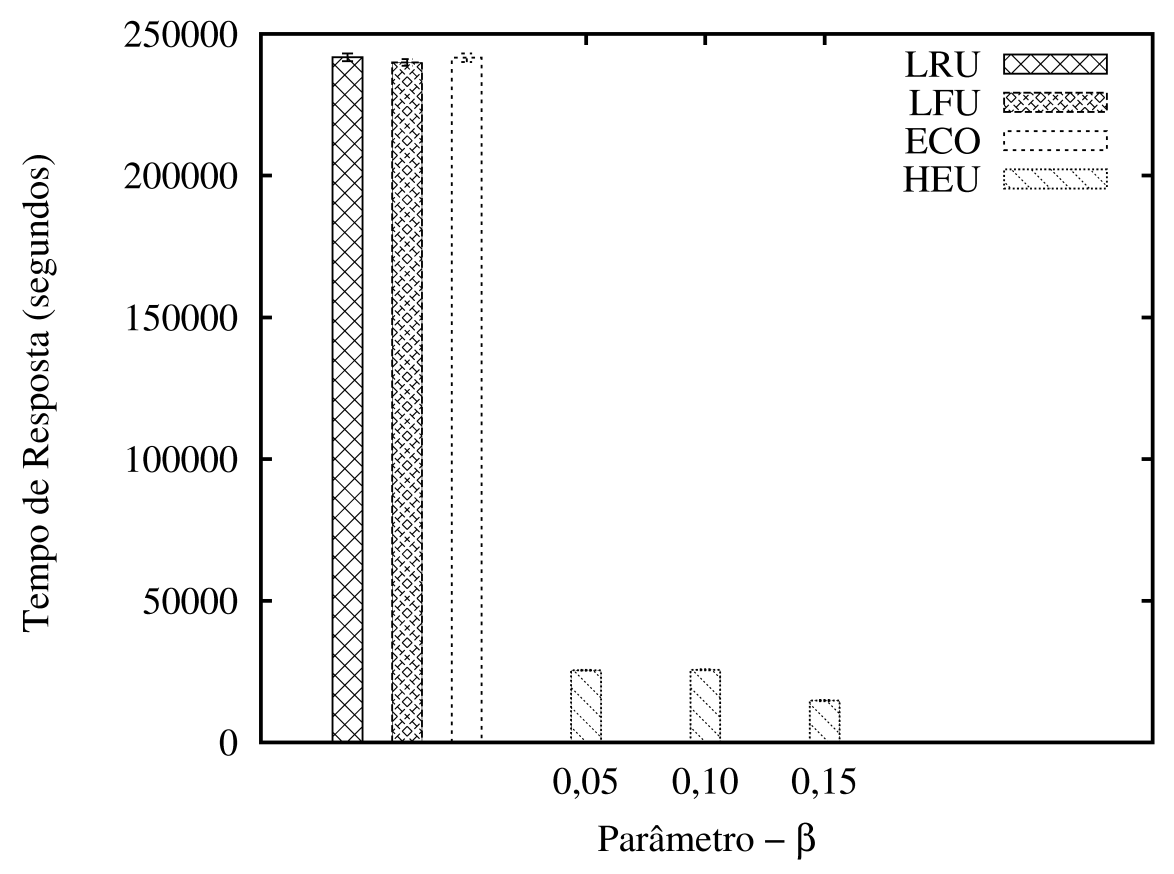

Figura 4.8: Resultados do experimento que considera um ambiente com $100 \%$ de operações de leitura.

Na Figura 4.8, o ambiente simulado tem $100 \%$ de operações de leitura e a quantidade de arquivos que um processo pode acessar é representada por uma função de distribuição de probabilidade uniforme com média 10. O eixo $x$ corresponde aos valores do parâmetro $\beta$, utilizado na heurística, e o 
eixo y representa o tempo de resposta médio dos processos (em segundos). Verifica-se no gráfico da Figura 4.8, que a heurística foi capaz de reduzir o tempo de resposta (execução) médio em uma ordem de magnitude, quando comparada às demais técnicas de replicação.

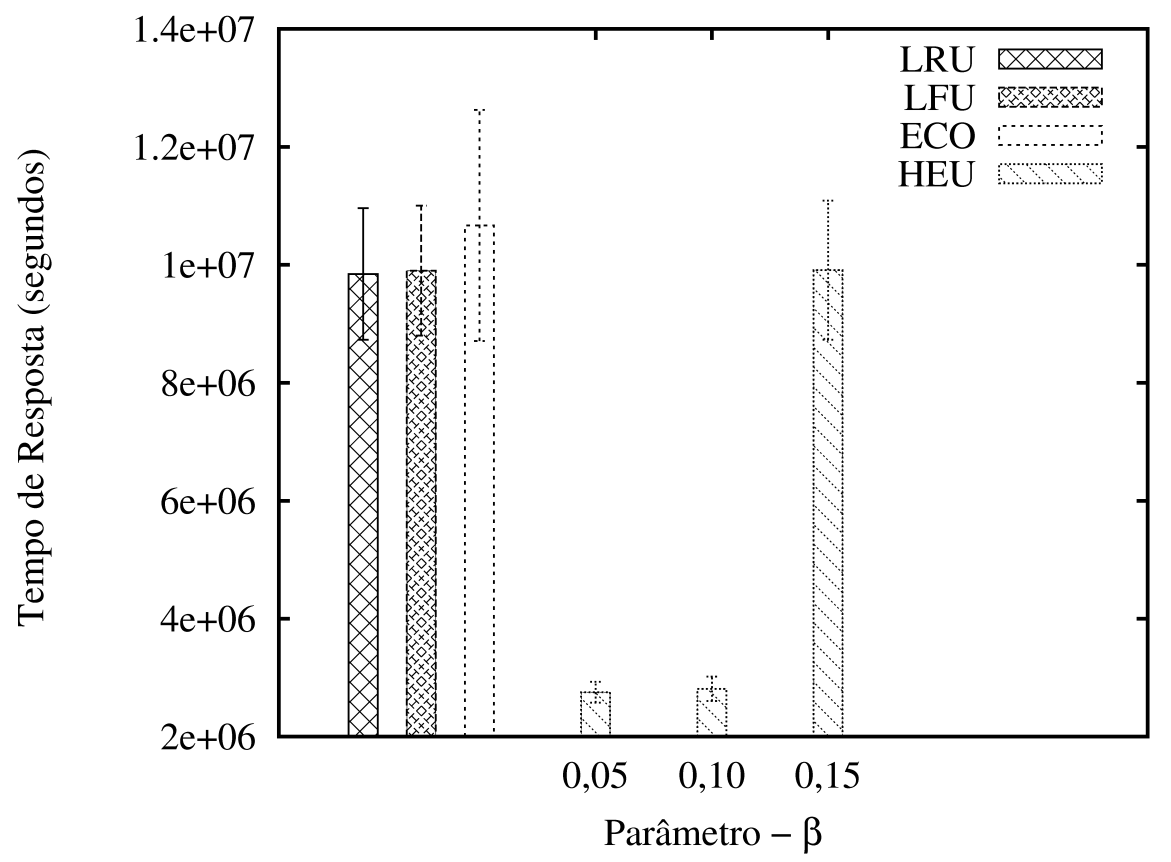

Figura 4.9: Resultados do experimento que considera um ambiente com $5 \%$ de operações de escrita e $95 \%$ de operações de leitura.

Na Figura 4.9, o ambiente tem 5\% de operações de escrita e $95 \%$ de operações de leitura. Além disso, a quantidade de arquivos que um processo pode acessar é a mesma do experimento anterior (função de distribuição de probabilidade uniforme com média 10). Neste cenário, a heurística reduziu o tempo de resposta em uma ordem de magnitude, entretanto, para um $\beta=0,15$, o resultado foi similar ao das demais técnicas avaliadas.

Na Figura 4.10, o ambiente simulado apresenta $95 \%$ de operações de escrita e $5 \%$ de operações de leitura. A quantidade de arquivos acessados por um processo é a mesma do experimento anterior. Neste experimento, a heurística reduziu o tempo de resposta dos processos em torno de $40 \%$. No entanto, para $\beta=0,15$, o resultado da heurística foi pior em comparação às outras técnicas avaliadas.

Os resultados obtidos com a heurística que adota janela adaptativa foram comparados à versão inicial que considera janelas deslizantes de tamanho fixo (proposta em (Ishii e de Mello, 2009)). Nessa comparação, a heurística com janela adaptativa apresentou desempenho similar em ambientes com $100 \%$ de operações de leitura, foi $10 \%$ melhor para ambientes com $5 \%$ de operações de escrita e $15 \%$ melhor para ambientes com $95 \%$ de operações de escrita, o que confirma a utilidade de uma janela que se adapta ao comportamento dos 


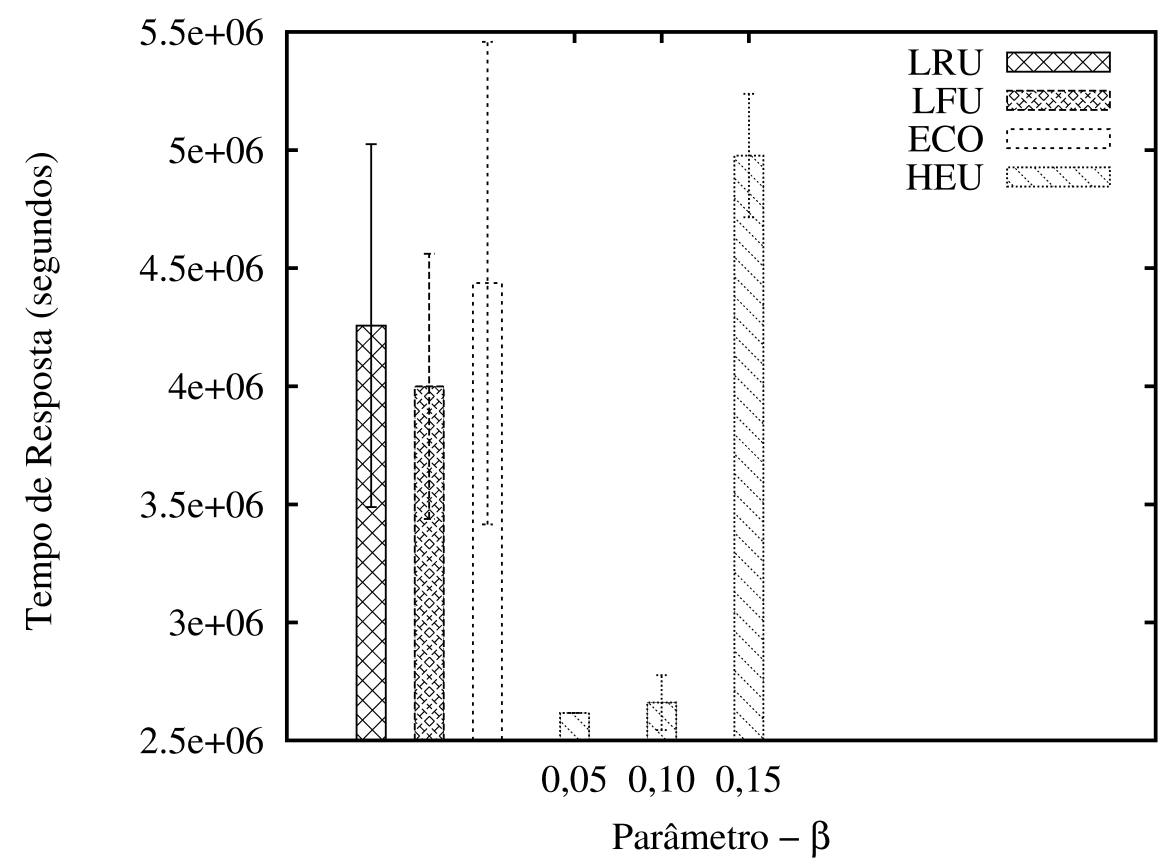

Figura 4.10: Resultados do experimento que considera um ambiente com $95 \%$ de operações de escrita e $5 \%$ de operações de leitura.

processos.

Esses resultados comprovam melhorias significativas no desempenho das aplicações e sugere a adoção de uma janela deslizante adaptativa de eventos futuros. Nota-se que a adaptação da janela é mais adequada para $\beta \in$ $[0,05 ; 0,10]$. Isso foi atestado experimentalmente, uma vez que $\beta \in[0,05 ; 0,10]$ é dada maior relevância à janela atual, $J_{t}$, de acordo com a Equação 4.9. No entanto, aumentando-se o valor de $\beta$, mais relevância é dada ao termo Op da Equação 4.9, ou seja, quanto menor é o valor de $\beta$, mais importância é dada ao comportamento dos processos dentro da janela atual e, consequentemente, melhor é o ajuste do comprimento da próxima janela.

A redução dos custos de acesso foi alcançada em razão do ajuste mais preciso da janela deslizante, ou seja, o comprimento da janela segue o comportamento dinâmico dos processos. Isso permite que a quantidade de eventos avaliados seja atualizada constante e precisamente, auxiliando na tomada de decisões mais elaboradas e relacionadas à replicação, migração e consistência de dados. Nota-se que a alta variabilidade no padrão de acesso, isto é, a intercalação entre diferentes tipos de operações - leitura, escrita e períodos de inatividade - proporciona instabilidade no desempenho das aplicações.

A partir dos resultados obtidos, comprova-se a eficiência da abordagem proposta, que emprega histórico de operações, técnicas de interceptação e janela deslizante. Entretanto, o uso de histórico de operações requer uma execução inicial da aplicação no sistema. Essa execução pode levar muito 
tempo, o que prejudica a abordagem. Além disso, essa abordagem também é prejudicada por aplicações com alta variação comportamental.

\subsection{Segunda abordagem proposta: predição on-line de comportamento de processos}

A primeira abordagem requer ao menos uma execução prévia da aplicação para composição de histórico. Esse requisito pode limitar aplicações reais que necessitam de longos períodos de execução para completar seu processamento, ou que apresentam mudanças comportamentais significativas. A fim de otimizar o acesso a dados sem considerar históricos (bases de conhecimento com informações das operações realizadas por processos de aplicações), foi proposta uma segunda abordagem que utiliza predição automática e on-line de acessos de leitura e escrita de processos. Essa abordagem emprega técnicas de extração de conhecimento (Subseção 4.4.1), predição (descritas na Seção 3.3) e janela deslizante adaptativa (Subseção 4.4.2). Além disso, ela usa a mesma heurística proposta no Algoritmo 5, entretanto, as informações consideradas são obtidas de forma preditiva e não histórica.

A Figura 4.11 descreve todas as etapas envolvidas nessa abordagem preditiva. Inicialmente, o comportamento dos processos é interceptado e capturado (nesse caso, quantidade de bytes, ver Tabela 4.5) e filtrado, transformando-o em uma série temporal para cada processo. Em seguida, essa série é avaliada considerando ferramentas específicas de análise: Recurrence Plot para identificação de estocasticidade e determinismo (Subseção 3.5.1), WNN para avaliação de linearidade (Subseção 3.5.2), STP e ACF para a verificação de estacionariedade (Subseção 3.5.3).

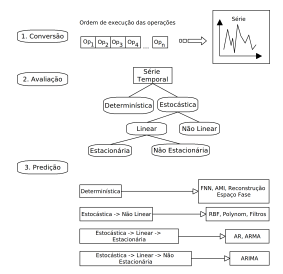

Figura 4.11: Etapas da abordagem baseada em predição on-line.

Após a classificação da série temporal por meio da metodologia proposta no Capítulo 3, obtém-se um modelo que melhor representa o processo gerador da série. A partir desse modelo, pode-se adotar diferentes técnicas para a predição de eventos futuros (as técnicas consideradas estão descritas na Seção 3.3). O mapeamento da classificação obtida para a técnica de predição é 
apresentado na Figura 4.11. Resultados desse mapeamento foram discutidos e validados na Seção 3.7.

Com o objetivo de validar a abordagem de otimização preditiva, foram conduzidas simulações no OptorSim. Uma nova estratégia de otimização de acesso a dados foi desenvolvida e incorporada ao simulador. Essa estratégia utiliza o Algoritmo 5, previamente definido, com a diferença que decisões de replicação, migração e consistência são tomadas com base em eventos preditos e não sobre informações históricas (Algoritmo 6).

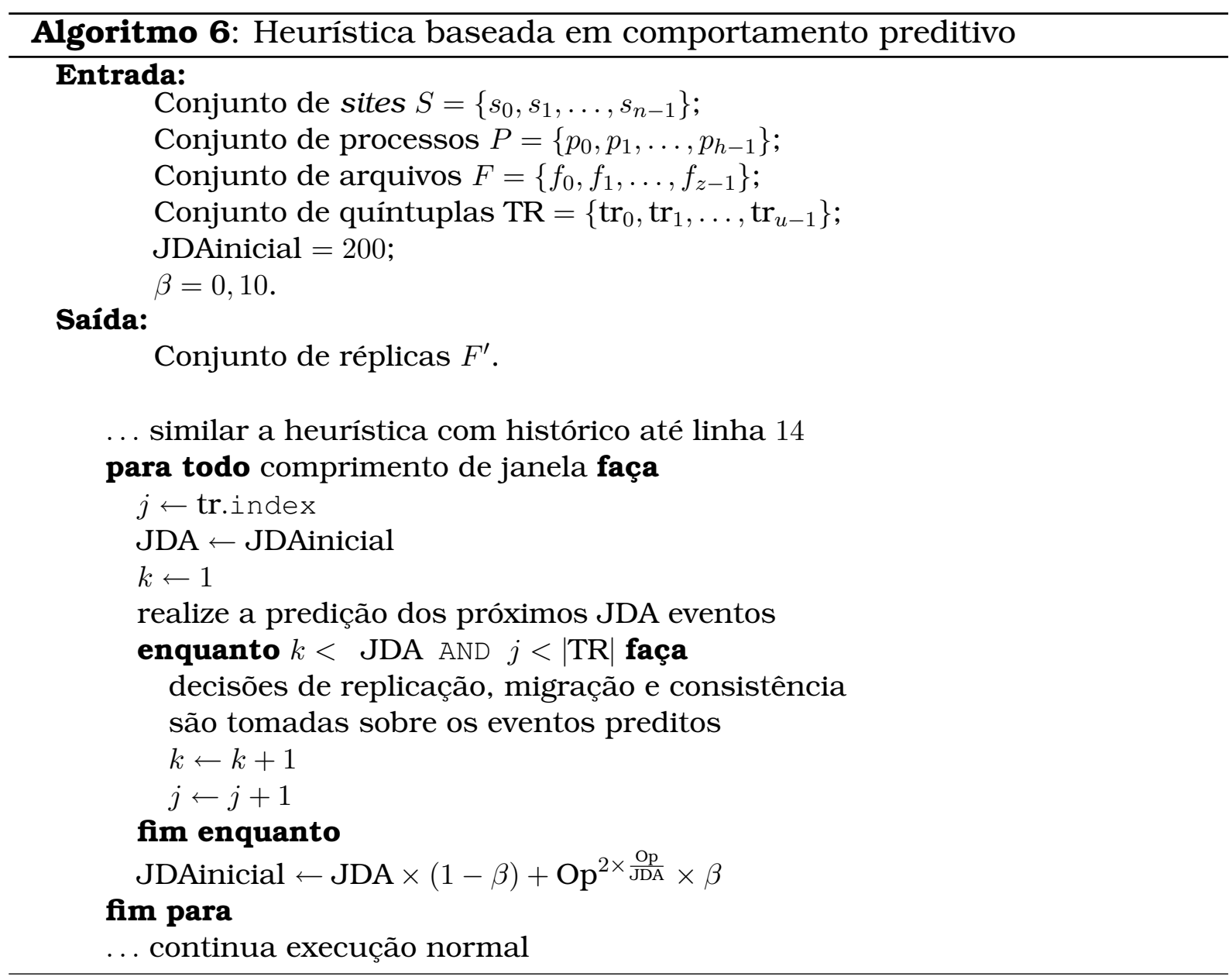

Considere como exemplo as informações apresentadas na Tabela 4.7. Assuma que o processo $p_{9}$ realiza somente operações de leitura sobre o arquivo $f_{1}$ nos intervalos determinados na primeira coluna da Tabela 4.7. A quantidade de bytes lidos é definida na segunda coluna e os valores preditos, na terceira. Dessa maneira, as decisões de replicação e migração são tomadas sob as informações da coluna 3, mas a execução real, ou seja, o processamento dos dados, é realizada sobre os dados reais (coluna 2). Essas decisões de replicação e migração consideram custos de transferência de dados entre os dispositivos de armazenamento do ambiente distribuído. Portanto, devem avaliar critérios como latência, largura de banda, capacidade de armazenamento, etc. Essas questões estão equacionadas nas definições 
apresentadas na Seção 4.3.

Tabela 4.7: Exemplo de informações utilizadas na otimização de acesso por meio da abordagem baseada em predição on-line.

\begin{tabular}{ccc} 
Intervalo & Quantidade Real de bytes & Quantidade Predita de bytes \\
\hline \hline 130 & 2000 & 1750 \\
1200 & 50 & 200 \\
900 & 300 & 280 \\
3 & 1000 & 800 \\
50 & 10 & 100 \\
2100 & 5 & 30 \\
450 & 2 & 1 \\
\hline
\end{tabular}

A técnica de janela deslizante adaptativa (JDA) também é utilizada nessa segunda abordagem. Nesse caso, a técnica de predição realiza a primeira estimativa somente após a execução de JDA eventos futuros, ou seja, há uma janela mínima de eventos a ser considerada a fim de predizer operações futuras de um processo. Os eventos preditos são, então, utilizados para a tomada de decisões de replicação, migração e consistência dos dados. Deve-se ressaltar que as decisões são tomadas com base nas predições, no entanto, o simulador OptorSim executa, efetivamente, as operações (leitura ou escrita em arquivo) reais e não as preditas.

\subsubsection{Resultados da segunda abordagem}

A fim de validar a abordagem preditiva, foram conduzidos experimentos utilizando o simulador OptorSim. Três conjuntos de dados, obtidos a partir do repositório SNIA, foram considerados: proj, hm e mds, todos definidos previamente na Tabela 3.12. O traço de execução proj é constituído somente de operações de escrita, enquanto os traços hm e mds, somente de operações de leitura.

Um exemplo esquemático de como é organizada a execução desta abordagem (incorporada no OptorSim) é apresentado na Figura 4.12.

No instante em que a primeira janela de eventos está completa, ou seja, a quantidade de operações executadas corresponde ao comprimento da janela (no Algoritmo 6 é definida uma janela inicial), o simulador realiza uma chamada de sistema ao pacote Tisean $^{3}$, passando as informações das operações presentes na janela atual. Com essas informações, o Tisean realiza a predição dos próximos JDA eventos, devolvendo-os para o OptorSim. O simulador continua sua execução, tomando decisões de replicação, migração e consistência segundo as regras da heurística proposta.

\footnotetext{
${ }^{3}$ Projeto para análise de séries temporais, o qual emprega métodos baseados em sistemas dinâmicos e teoria do caos (Hegger et al., 1998).
} 


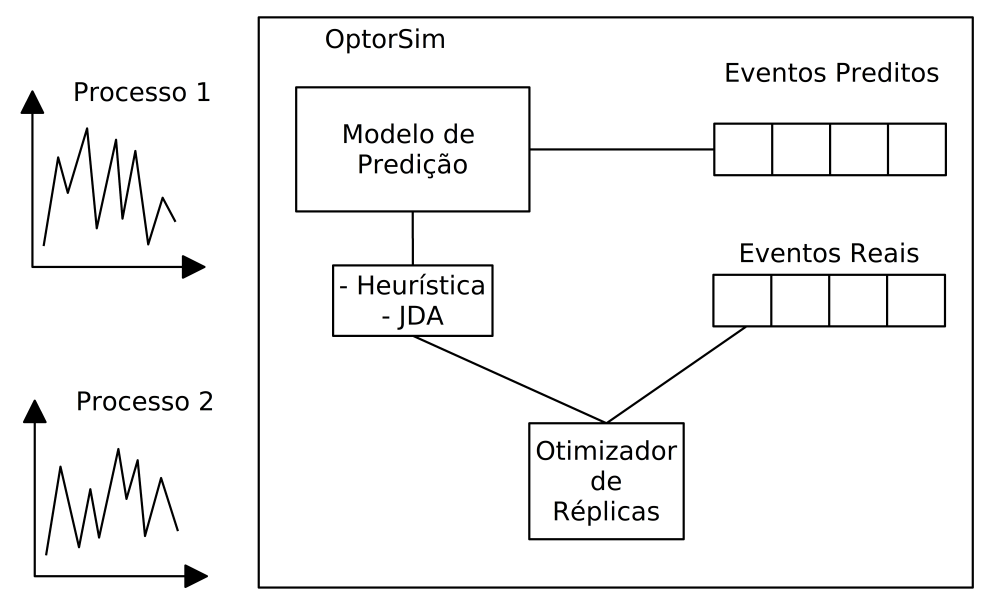

Figura 4.12: Exemplo de execução baseada em predição on-line.

Foram avaliadas as técnicas de otimização de acesso a dados disponíveis no OptorSim, bem como a heurística baseada em histórico: LRU, LFU, Modelo Econômico (ECO), Heurística proposta com uso de histórico (H-Hist) e Heurística proposta com uso de predição (H-Pred).

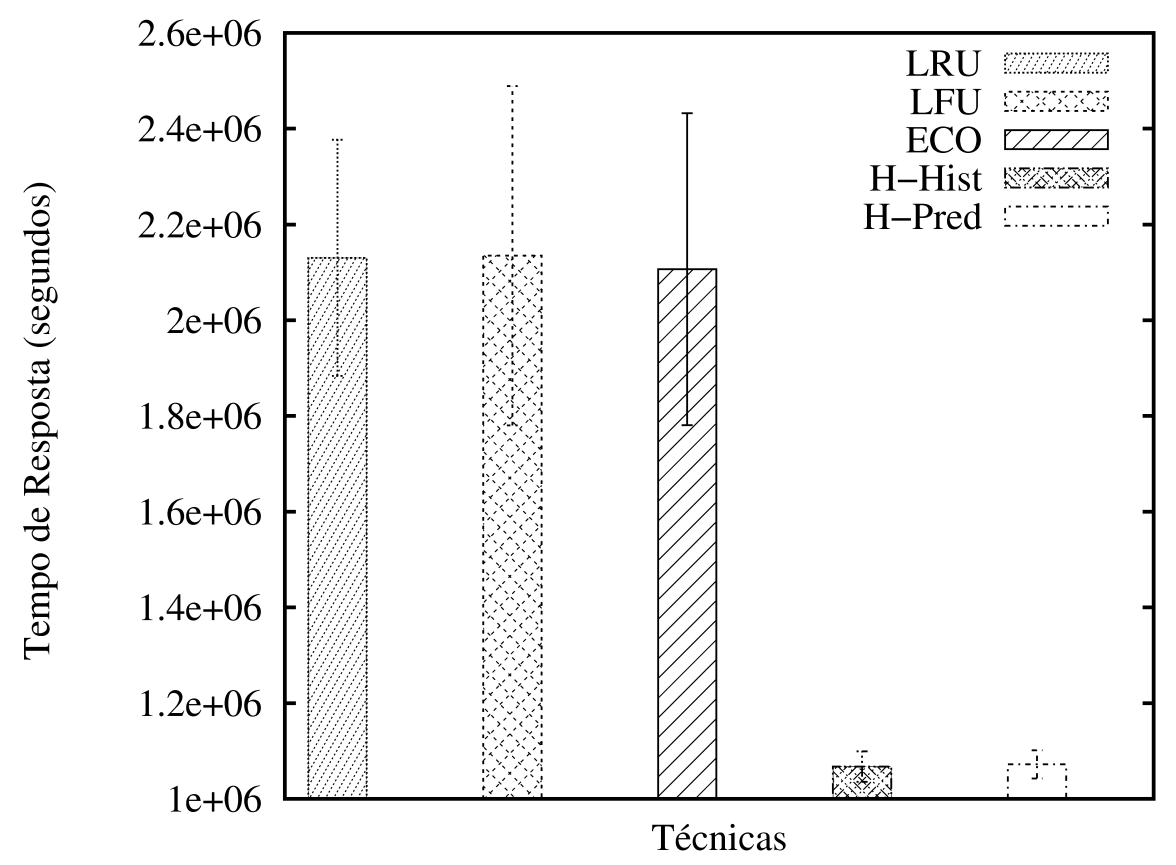

Figura 4.13: Resultados comparativos entre as técnicas de otimização LRU, LFU, ECO, H-Hist e H-Pred para o traço de execução hm.

O primeiro experimento avalia o desempenho das técnicas de otimização sobre o traço de execução hm. A métrica considerada é o tempo de resposta (execução) médio (em segundos). Por meio da Figura 4.13, nota-se que a heurística (H-Hist) foi capaz de reduzir o tempo de resposta em $60 \%$ quando comparada às demais técnicas. Além disso, a heurística (H-Pred), que emprega a técnica ARIMA $(2,1,2)$ para predição de operações, tem desempenho equivalente ao da técnica com histórico. 


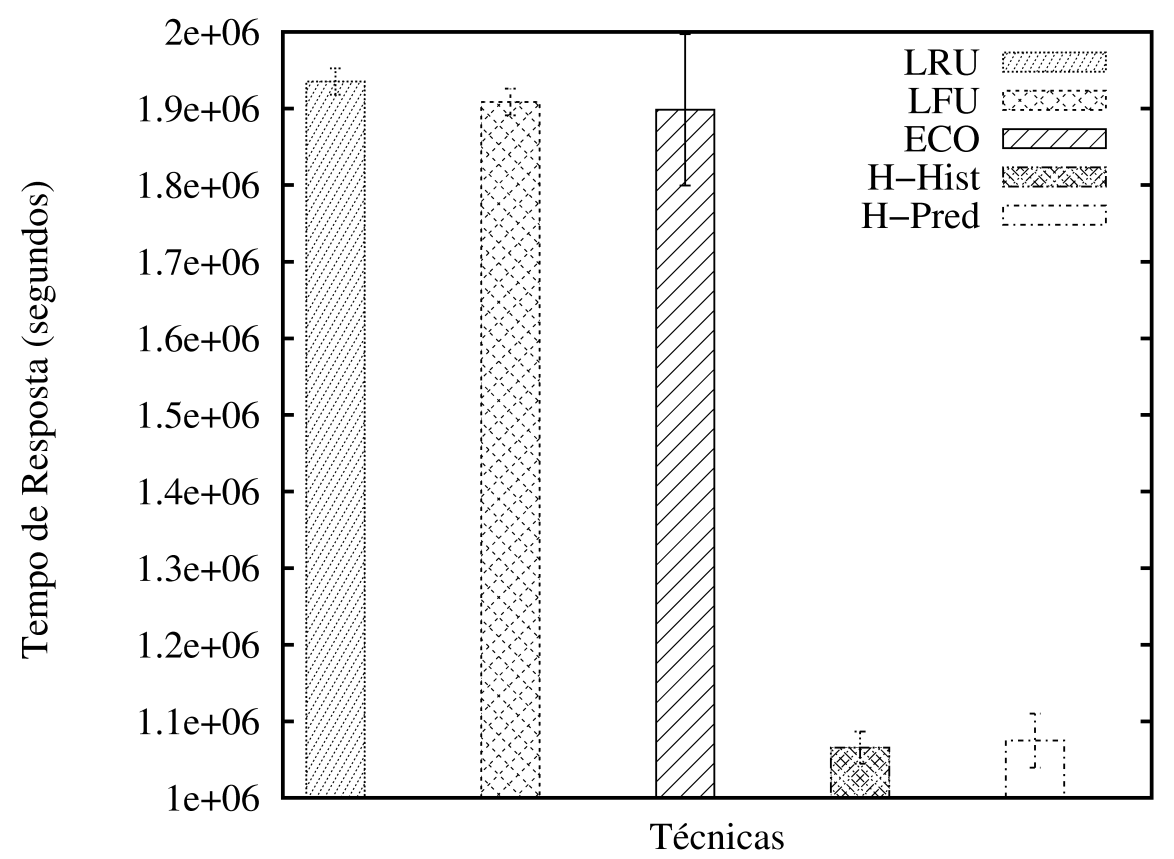

Figura 4.14: Resultados comparativos entre as técnicas de otimização LRU, LFU, ECO, H-Hist e H-Pred para o traço de execução mds.

O próximo experimento avalia o desempenho das técnicas de otimização, considerando um ambiente que adota o traço de execução mds com somente operações de leitura. Observa-se na Figura 4.14 que a técnica H-Hist foi capaz de reduzir o tempo médio de resposta em $57 \%$ quando comparada às demais técnicas. Além disso, a heurística (H-Pred), que emprega a técnica Espaço Fase/RBF para predição de operações, tem o mesmo desempenho da técnica com histórico.

O próximo experimento avalia o desempenho das técnicas de otimização, considerando um ambiente que adota o traço de execução proj para modelagem das operações de escrita. Na Figura 4.15, a técnica H-Hist tem desempenho inferior à melhor técnica de otimização, ECO, em $2 \approx 3 \%$. Além disso, a heurística H-Pred, que emprega a técnica $\operatorname{ARMA}(1,2)$ para predição de operações, tem desempenho $3 \approx 4 \%$ inferior à técnica ECO.

Os resultados do último conjunto de dados, traço proj, motivaram uma investigação em busca das causas do desempenho reduzido (Figura 4.15). O traço proj tem somente operações de escrita, o que implica em muitas atualizações, podendo indicar deficiência no algoritmo de consistência. A fim de confirmar essa hipótese foi realizado outro experimento sobre um conjunto de dados modificado. O tipo das operações foi alterado artificialmente, ou seja, alguns eventos do traço proj tiveram o valor do campo op modificado para leitura. Os eventos que sofreram essa modificação foram os seguintes: $\{10,100,200,300,400,500,1.000,1.500,2.000\}$.

Nota-se por meio da Figura 4.16 que H-Hist tem desempenho similar 


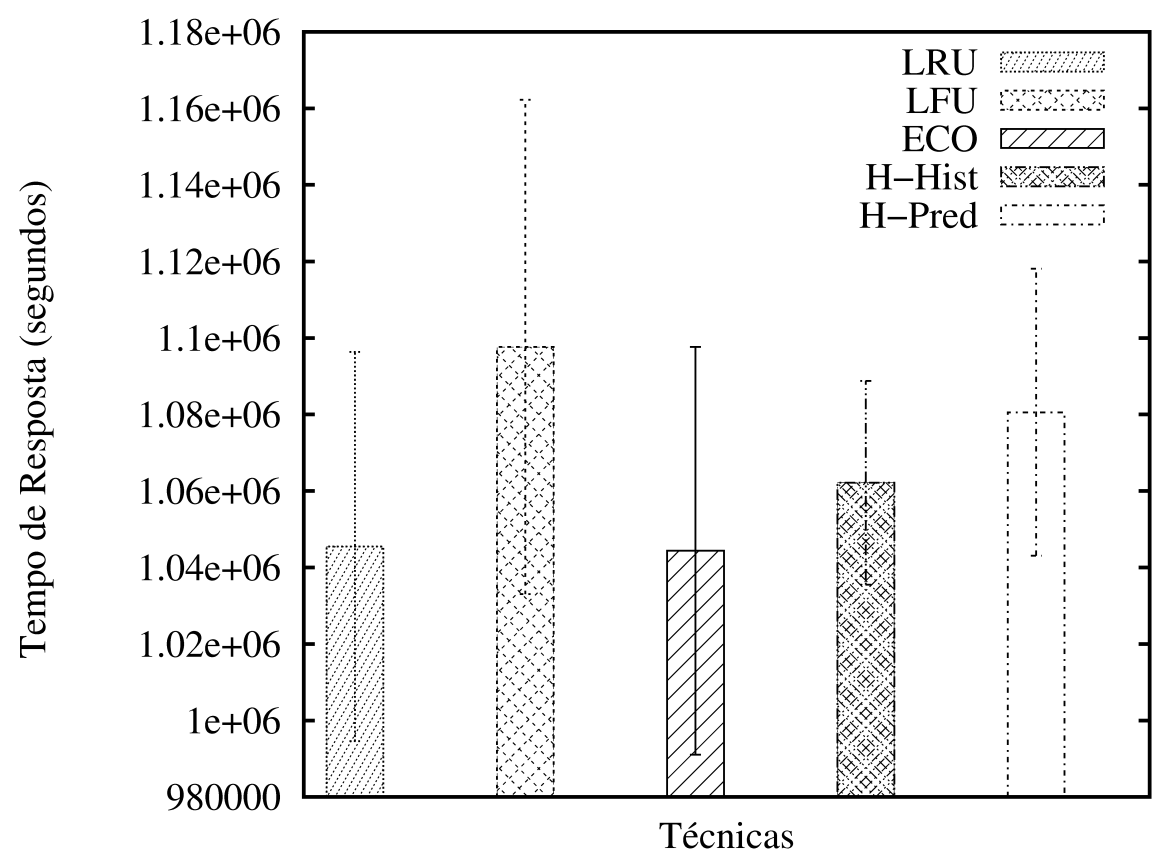

Figura 4.15: Resultados comparativos entre as técnicas de otimização LRU, LFU, ECO, H-Hist e H-Pred para o traço de execução proj.

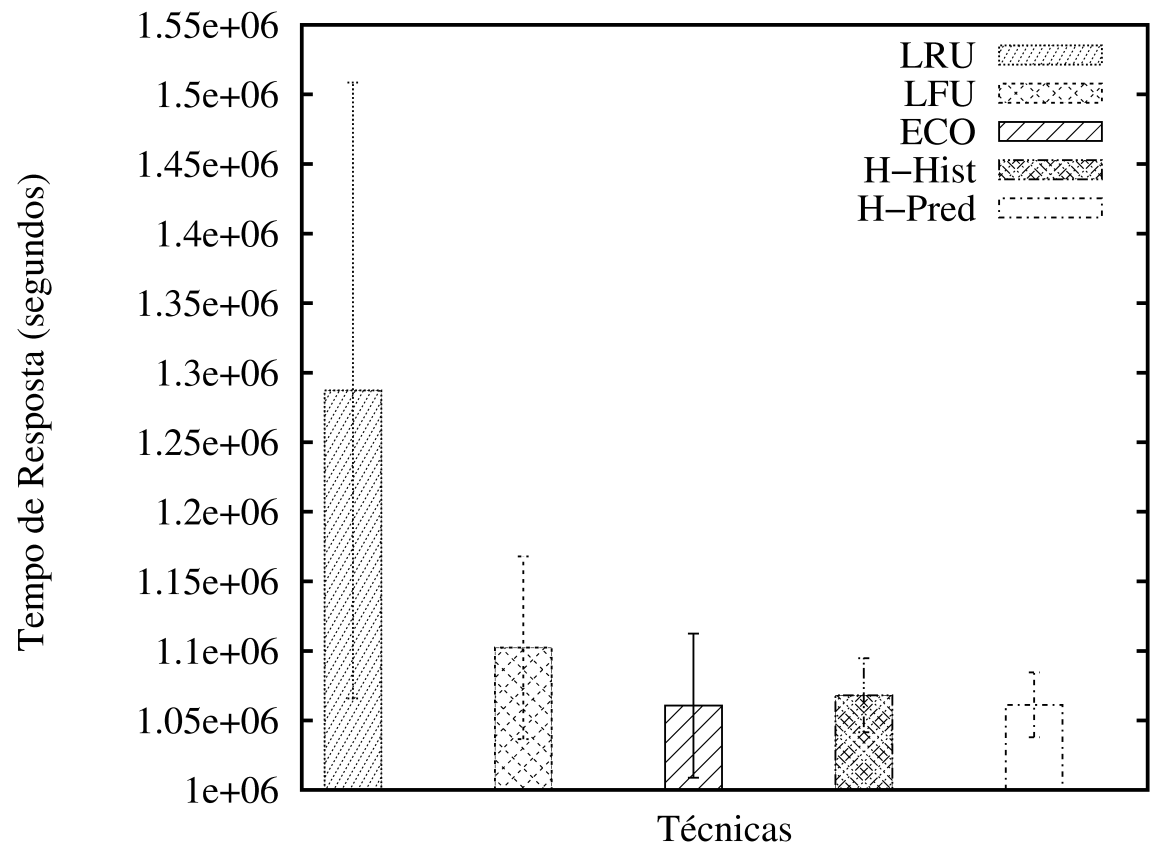

Figura 4.16: Resultados comparativos entre as técnicas de otimização LRU, LFU, ECO, H-Hist e H-Pred para o traço de execução proj com modificações. 
à melhor técnica de otimização, ECO, para esse traço de execução. Esse resultado confirma a hipótese da deficiência do mecanismo de consistência de dados que, na atual implementação, considera uma abordagem sequencial (em fila) para a atualização de réplicas dos arquivos. Outros mecanismos podem ser avaliados, tal como o Lazy Consistency (Sun e Xu, 2004), que permite um controle de coerência mais flexível e tende a fornecer desempenho superior (Ladin et al., 1992).

A segunda abordagem apresenta resultados equivalentes ou, em alguns casos (Figura 4.14), inferiores aos da abordagem baseada em histórico, conforme demonstrado na Subseção 4.5.1. No entanto, essa abordagem preditiva não requer uma execução inicial da aplicação, evita o armazenamento de longos históricos e adapta-se, eficientemente, às variações comportamentais de aplicações. Isso amplia a diversidade de uso da heurística proposta, que pode otimizar operações de acesso a dados além das restrições impostas pela abordagem histórica.

Além disso, observa-se, em todos os gráficos desta seção, que, além de aumentar o desempenho de aplicações, as abordagens histórica e preditiva apresentam baixa variabilidade no tempo de resposta, quando comparadas às demais técnicas de otimização, ou seja, apresentam baixo desvio padrão. Essa característica torna ambas abordagens mais confiáveis sob diferentes cenários, o que reforça o emprego de conhecimento sobre aplicações a fim de otimizar operações de acesso a dados.

\subsection{Considerações Finais}

Este capítulo apresentou as duas abordagens propostas nesta tese para o problema de acesso a dados. Na primeira, uma heurística avalia e toma decisões de replicação, migração e consistência de dados segundo uma janela deslizante adaptativa de eventos obtidos a partir de históricos comportamentais de aplicações. Nesse caso, aplicações necessitam de uma execução inicial para a extração dessas informações. Na segunda abordagem, essas informações são extraídas em tempo de execução (on-line), e, por meio de análise de séries temporais, encontra-se o modelo mais adequado para predizer eventos futuros de acesso, os quais são empregados pela mesma heurística.

Os resultados de ambas as abordagens demonstram que a antecipação de operações de leitura e escrita aumenta o desempenho de aplicações data-intensive, atestando a hipótese desta tese. Além disso, cabe ressaltar que a segunda abordagem amplia a diversidade de uso da heurística proposta, otimizando operações de acesso a dados além das restrições impostas pela 
abordagem histórica. 


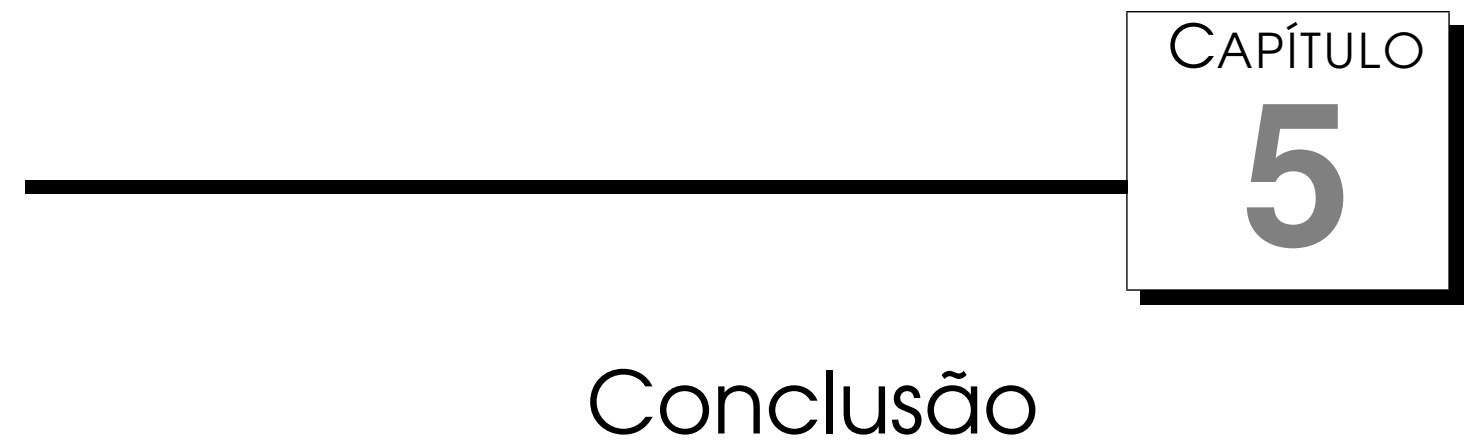

Este capítulo apresenta as conclusões desta tese, discutindo as principais contribuições, dificuldades encontradas e propostas para trabalhos futuros.

\subsection{Conclusões}

Aplicações data-intensive tornaram-se o foco de muitas pesquisas na área de processamento de alto desempenho (Oliker et al., 2004; Sun e Xu, 2004; Rahman et al., 2005; Wang et al., 2006; Oldfield e Kotz, 2006; Dang e Lim, 2007; Elghirani et al., 2007; Sato et al., 2009; Kim et al., 2009; Chervenak et al., 2009; AL-Mistarihi e Yong, 2009). Essas pesquisas visam a melhoria do acesso às informações, ou seja, o desenvolvimento de mecanismos que reduzam o tempo de resposta e aumentem a vazão de sistemas. Entretanto, a maioria desses trabalhos não emprega nem utiliza informações sobre o comportamento de aplicações para realizar otimizações de acesso. Esse fato motivou esta tese que considera características comportamentais (ou conhecimento) dos processos a fim de tomar decisões relativas à replicação, migração e consistência de dados.

O uso do conhecimento sobre o comportamento de processos requer duas etapas: extração e avaliação. A extração pode ser obtida por meio de duas abordagens: monitoração e interceptação (Dodonov e de Mello, 2010). Experimentos conduzidos e discutidos na Subseção 4.4.7 demonstram que a técnica de interceptação é a mais adequada para a extração de conhecimento de operações de entrada e saída em ambientes reais.

Para a avaliação do conhecimento, duas abordagens foram propostas: na primeira, uma heurística é utilizada para avaliar comportamento histórico 
de processo, enquanto, na segunda, a mesma heurística considera eventos preditos. A heurística baseada em histórico toma suas decisões de otimização de acesso por meio de uma janela deslizante adaptativa. Essa janela tem informações sobre os eventos (Tabela 4.5) e, além disso, permite o ajuste das decisões segundo o comportamento atual dos processos. Os experimentos apresentados na Subseção 4.4.8 demonstram a redução no tempo de resposta de aplicações, quando comparada a outras técnicas comumente empregadas na literatura.

Entretanto, essa primeira abordagem baseada em histórico necessita de uma execução prévia da aplicação, o que nem sempre é praticável, principalmente tratando-se de aplicações científicas que podem demorar longos períodos para execução completa. Além disso, aplicações que apresentam mudanças comportamentais significativas podem prejudicar boa estimativas. Por fim, a necessidade de armazenamento de informações históricas pode se tornar onerosa para o desempenho da abordagem.

Essas limitações motivaram a proposta de uma segunda abordagem, a qual emprega predição on-line de eventos de leitura e escrita. Para que a predição tenha resultados satisfatórios, as operações sobre os dados são transformadas em observações de séries temporais, avaliadas segundo critérios de estocasticidade, linearidade e estacionariedade (Capítulo 3). Em seguida, essas séries são classificadas e os modelos que melhor as representam são obtidos. A partir desses modelos, técnicas de predição são aplicadas para caracterizar eventos futuros.

A segunda abordagem apresenta resultados equivalentes ou, em alguns casos (Figura 4.14), inferiores aos da abordagem baseada em histórico, conforme demonstrado na Subseção 4.5.1. No entanto, essa abordagem preditiva não requer uma execução inicial da aplicação, evita o armazenamento de longos históricos e adapta-se, eficientemente, às variações comportamentais de aplicações. Isso amplia a diversidade de uso da heurística proposta, que pode otimizar operações de acesso a dados além das restrições impostas pela abordagem histórica.

Em ambas abordagens, as heurísticas reduzem o tempo de resposta médio em aproximadamente 50\%, o que comprova a hipótese desta tese, ou seja, a antecipação de operações de leitura e escrita em arquivos aumenta o desempenho de aplicações data-intensive em ambientes computacionais de larga escala.

\subsection{Contribuições}

As principais contribuições desta tese são: 
1. proposta de uma metodologia para a análise de séries temporais sem a necessidade de conhecimento prévio sobre seus processos geradores. Essa metodologia adota testes de aderência para verificar níveis de estocasticidade, linearidade e estacionariedade, com objetivo de obter modelos mais adequados ao comportamento dos dados (Capítulo 3);

2. definição formal do problema de acessos a dados (PAD) e prova de sua NP-Completude (Seção 4.3);

3. definição de um modelo analítico para o PAD que visa minimizar o tempo de resposta médio de aplicações que manipulam grandes volumes de dados (Seção 4.3);

4. proposta de uma heurística que considera histórico de informações (Seção 4.4);

5. definição de uma janela deslizante adaptativa que considera eventos futuros (operações de leitura e escrita) para otimizar o acesso a dados (Subseção 4.4.2);

6. adaptação da heurística proposta para utilizar informações preditas a fim de otimizar o acesso a dados (Seção 4.5).

\subsubsection{Relação com demais trabalhos do grupo de pesquisa}

Trabalhos prévios ao desenvolvimento desta tese investigaram diversos mecanismos para a modelagem e predição de comportamento de aplicações, buscando otimizar o escalonamento de processos em ambientes de larga escala (de Mello, 2009; Dodonov, 2009). Além desses trabalhos, há ainda outros em desenvolvimento, que abordam problemas correlacionados:

1. Iniciação científica: implementação de técnicas de replicação, migração e consistência de dados em um sistema de arquivo distribuído real;

2. Mestrado: emprego de conhecimento sobre aplicações com objetivo de detectar faltas durante a execução de processos;

3. Mestrado: emprego de conhecimento sobre aplicações com objetivo de detectar intrusão e ataques maliciosos em sistemas reais;

4. Doutorado: proposta de uma rede neural para a detecção de novidades em séries temporais;

5. Doutorado: estudos para a identificação de propriedades e características de séries temporais com o objetivo de aumentar a acurácia de modelagem; 
6. Doutorado: comportamento de processos e séries temporais aplicados ao escalonamento de processos.

\subsection{Dificuldades e principais problemas}

Uma das etapas desta pesquisa focou na avaliação de abordagens para otimização: algoritmos exatos e meta-heurísticas. Algoritmos exatos garantem uma solução ótima para um problema mas podem consumir muito tempo de execução e, portanto, tornam-se inviáveis para a tomada de decisões on-line, algo necessário neste contexto. Dada essa limitação, as meta-heurísticas Algoritmo Genético (AG), Ant Colony Optimization (ACO), Simulated Annealing (SA) e Redes Neurais de Hopfield (RN) foram avaliadas (Apêndice A). Em de Mello et al. (2007) as técnicas AG e RN foram empregadas no problema de distribuição de trechos (chunks) de arquivos com objetivo de maximizar a vazão no acesso aos dados. Em Ishii et al. (2007) a técnica SA foi utilizada para escalonamento de processos em grades computacionais. Em Souza et al. (2006) uma variação da abordagem ACO, denominada Particle Swarm Optimization (PSO) foi empregada para seleção de parâmetros em problemas multiclasse Support Vector Machine (SVM).

Entretanto, para o problema de acesso a dados, é fundamental que a técnica de otimização seja eficiente na modelagem do comportamento dinâmico de processos, mais especificamente, em operações de leitura e escrita. Esse fato não foi considerado pelos trabalhos anteriormente descritos (Souza et al., 2006; Ishii et al., 2007; de Mello et al., 2007), tampouco nos estudos iniciais desta tese, utilizando meta-heurísticas. Nessas abordagens, geralmente, emprega-se uma função objetivo que avalia informações de acesso no instante atual de execução, o que tende a otimizar em termos do comportamento médio de eventos. Dessa maneira, soluções mais eficientes deveriam avaliar alterações comportamentais e antecipar operações de acesso tal como proposto nesta tese (Capítulo 4).

Outra dificuldade está na grande divergência dos trabalhos sobre análise de séries temporais, principalmente, na avaliação de dados experimentais (nesta tese foram avaliados conjuntos de dados reais obtidos do repositório SNIA (Narayanan et al., 2008)). Essa questão motivou a proposta de uma metodologia para a classificação de séries temporais originárias de dados experimentais, ou seja, sem conhecimento prévio de seus processos geradores (Capítulo 3). 


\subsection{Propostas para trabalhos futuros}

Os resultados obtidos nesta tese motivam, principalmente, os seguintes trabalhos futuros: a implementação da heurística (Algoritmo 5 e de sua variante Algoritmo 6) em ambientes reais de produção, considerando sistemas de arquivos distribuídos; a proposta de diferentes algoritmos para a atualização de réplicas e manutenção de consistência de dados; a investigação de outras técnicas de modelagem, além daquelas descritas na Seção 3.3, a fim de confirmar a metodologia ou estendê-la a outros problemas; a utilização e a avaliação da metodologia de análise de séries temporais, proposta nesta tese, em outros trabalhos relacionados do grupo de pesquisa BIOCOM. 


\section{Referências Bibliográficas}

Abramovici, A.; Althouse, W. E.; Drever, R. W. P.; GÃ $\frac{1}{4}$ rsel, Y.; Kawamura, S.; Raab, F. J.; Shoemaker, D.; Sievers, L.; Spero, R. E.; Thorne, K. S.; Vogt, R. E.; Weiss, R.; Whitcomb, S. E.; Zucker, M. E. (1992). LIGO: The Laser Interferometer Gravitational-Wave Observatory. Science, v.256, n.5055, p.325-333. Citado na página 9.

AL-Mistarihi, H. H. E.; Yong, C. H. (2009). On fairness, optimizing replica selection in data grids. IEEE Trans. Parallel Distrib. Syst., v.20, n.8, p.1102-1111. Citado nas páginas 4, 10, 84, e 101.

Albert, R.; Barabasi, A.-L. (2001). Statistical mechanics of complex networks. Reviews of Modern Physics, v.74, p.47-101. Citado na página 85.

Albertini, M. K.; de Mello, R. F. (2007). A self-organizing neural network for detecting novelties. SAC '07: Proceedings of the 2007 ACM symposium on Applied computing, p. 462-466, New York, NY, USA. ACM. Citado na página 13.

Anderson, M.; Woessner, W. (1992). Applied Groundwater Modeling: Simulation of Flow and Advective Transport. Academic Press., 2nd edição. Citado na página 56.

Back, T.; Fogel, D. B.; Michalewicz, Z. (1999a). Advanced Algorithms and Operators. IOP Publishing Ltd., Bristol, UK, UK. Citado na página 119.

Back, T.; Fogel, D. B.; Michalewicz, Z. (1999b). Basic Algorithms and Operators. IOP Publishing Ltd., Bristol, UK, UK. Citado na página 119.

Bell, W. H.; Cameron, D. G.; Millar, A. P.; Capozza, L.; Stockinger, K.; Zini, F. (2003). Optorsim: A Grid Simulator for Studying Dynamic Data Replication Strategies. International Journal of High Performance Computing Applications, v.17, n.4, p.403-416. Citado nas páginas 4, 6, 10, e 84. 
Bonabeau, E.; M., D.; Theraulaz, G. (2000). Inspiration for optimization from social insect behaviour. Nature, v.406, n.6791, p.39-42. Citado na página 120.

Box, G.; Jenkins, G. M.; Reinsel, G. (1994). Time Series Analysis: Forecasting \& Control. Prentice Hall, 3rd edição. Citado nas páginas 18 e 20.

Bray, T. (2009). Bonnie benchmark. http://www.textuality.com/bonnie/. Citado na página 88.

Burtscher, M.; Sam, N. B. (2005). Automatic generation of high-performance trace compressors. CGO '05: Proceedings of the international symposium on Code generation and optimization, p. 229-240, Washington, DC, USA. IEEE Computer Society. Citado na página 9.

Casdagli, M. (1989). Nonlinear prediction of chaotic time series. Physica D: Nonlinear Phenomena, v.35, n.3, p.335-356. Citado na página 24.

Chapin, S. J.; Katramatos, D.; Karpovich, J.; Grimshaw, A. S. (1999). The Legion resource management system. Feitelson, D. G.; Rudolph, L., editores, Job Scheduling Strategies for Parallel Processing, p. 162-178. Springer Verlag. Citado na página 85.

Chatfield, C. (2004). The Analysis of Time Series: An Introduction. CRC Press LLC. Citado na página 27.

Chervenak, A. L.; Schuler, R.; Ripeanu, M.; Amer, M. A.; Bharathi, S.; Foster, I.; Iamnitchi, A.; Kesselman, C. (2009). The globus replica location service: Design and experience. IEEE Trans. Parallel Distrib. Syst., v.20, n.9, p.1260-1272. Citado nas páginas 4, 9, e 101.

Chun, B.; Culler, D.; Roscoe, T.; Bavier, A.; Peterson, L.; Wawrzoniak, M.; Bowman, M. (2003). Planetlab: an overlay testbed for broad-coverage services. SIGCOMM Comput. Commun. Rev., v.33, n.3, p.3-12. Citado na página 9.

Cochrane, G.; Akhtar, R.; Bonfield, J.; Bower, L.; Demiralp, F.; Faruque, N.; Gibson, R.; Hoad, G.; Hubbard, T.; Hunter, C.; Jang, M.; Juhos, S.; Leinonen, R.; Leonard, S.; Lin, Q.; Lopez, R.; Lorenc, D.; McWilliam, H.; Mukherjee, G.; Plaister, S.; Radhakrishnan, R.; Robinson, S.; Sobhany, S.; Hoopen, P. T.; Vaughan, R.; Zalunin, V.; Birney, E. (2009). Petabyte-scale innovations at the European Nucleotide Archive. Nucleic Acids Research, v.37, p.D19-D25. Citado na página 65.

Collins, J. P. (2010). Sailing on an ocean of 0s and 1s. Science, v.327, p.1455-1456. Citado nas páginas 1, 2, e 3. 
Corporation, S. P. E. (2010). SPEC's CPU Benchmark. See the definition of CINT2000. http://www.spec.org/. Citado na página 87.

Dang, N. N.; Lim, S. B. (2007). Combination of replication and scheduling in data grids. International Journal of Computer Science and Network Security, v.7, n.3, p.304-308. Citado nas páginas 3, 4, 8, 82, e 101.

de Mello, R. F. (2009). Sistemas Dinâmicos e Técnicas Inteligentes para a Predićão de Comportamento de Processos: Uma Abordagem para Otimizaćão de Escalonamento em Grades Computacionais. Tese (Doutorado), Intituto de Ciências Matemáticas e de Computaćão - USP. Citado nas páginas 4, 22, e 103.

de Mello, R. F.; Filho, J. A. A.; Dodonov, E.; Ishii, R. P.; Yang, L. T. (2007). Optimizing Distributed Data Access in Grid Environments by Using Artificial Intelligence Techniques. Lecture Notes in Computer Science, v. 4742/2007, p. 125-136. Springer Berlin / Heidelberg. Citado nas páginas 73 e 104.

Deelman, E.; Blythe, J.; Gil, Y.; Kesselman, C.; Mehta, G.; Vahi, K.; Blackburn, K.; Lazzarini, A.; Arbree, A.; Cavanaugh, R.; Koranda, S. (2003). Mapping Abstract Complex Workflows onto Grid Environments. Journal of Grid Computing, v.1, n.1, p.25-39. Citado na página 9.

Deneubourg, J. L.; Goss, S.; Franks, N.; Sendova-Franks, A.; Detrain, C.; Chrétien, L. (1990). The dynamics of collective sorting robot-like ants and ant-like robots. Proceedings of the first international conference on simulation of adaptive behavior on From animals to animats, p. 356-363, Cambridge, MA, USA. MIT Press. Citado na página 121.

Diks, C.; van Houwelingen, J. C.; Takens, F.; DeGoede, J. (1995). Reversibility as a criterion for discriminating time series. Physics Letters A, v.201, n.2-3, p.221 - 228. Citado na página 19.

Dodonov, E. (2009). Sistemas Dinâmicos e Técnicas Inteligentes para a Predićão de Comportamento de Processos: Uma Abordagem para Otimizaćão de Escalonamento em Grades Computacionais. Tese (Doutorado), Instituto de Ciências Matemáticas e de Computaćão - USP. Citado nas páginas 4 e 103.

Dodonov, E.; de Mello, R. F. (2010). A novel approach for distributed application scheduling based on prediction of communication events. Future Generation Comp. Syst., v.26, n.5, p.740-752. Citado nas páginas 4, 13, e 101. 
Dorigo, M.; Di Caro, G. (1999). The ant colony optimization meta-heuristic. Corne, D.; Dorigo, M.; Glover, F., editores, New Ideas in Optimization, p. 11-32. McGraw-Hill, London. Citado nas páginas 5, 73, e 120.

Elghirani, A.; Subrata, R.; Zomaya, A. Y. (2007). Intelligent scheduling and replication in datagrids: a synergistic approach. Proc. $7^{\text {th }}$ IEEE Int.Symposium on Cluster Computing and the Grid, p. 179-182, Washington, DC, USA. Citado nas páginas 3, 4, 9, e 101.

Faerman, M.; Su, A.; Wolski, R.; Berman, F. (1999). Adaptive performance prediction for distributed data-intensive applications. Supercomputing '99: Proceedings of the 1999 ACM/IEEE conference on Supercomputing (CDROM), p. 36, New York, NY, USA. ACM. Citado na página 10.

Feng, J.; Humphrey, M. (2004). Eliminating Replica Selection - Using Multiple Replicas to Accelerate Data Transfer on Grids. Proc. $10^{\text {th }}$ Int. Conf. of Parallel and Distributed Systems, p. 359, Washington, DC, USA. IEEE Computer Society. Citado na página 8.

Ferrer-i-Cancho, R.; Sole, R. V. (2002). Zipf's law and random texts. Advances in Complex Systems, v.5, n.1, p.1-6. Citado na página 85.

Fox, G.; Gannon, D. (2001). Computational grids. Computing in Science and Engg., v.3, n.4, p.74-77. Citado na página 3.

Francis, J. G. F. (1961). The QR Transformation a Unitary Analogue to the LR Transformation - Part 1. The Computer Journal, v.4, n.3, p.265-271. Citado na página 24.

Fraser, A. M.; Swinney, H. L. (1986). Independent coordinates for strange attractors from mutual information. Phys. Rev. A, v.33, n.2, p.1134-1140. Citado nas páginas 22, 23, e 27.

Galka, A.; Ozaki, T. (2001). Testing for nonlinearity in high-dimensional time series from continuous dynamics. Phys. D, v.158, n.1-4, p.32-44. Citado na página 19.

Gallant, A. R.; White, H. (1992). Original contribution: On learning the derivatives of an unknown mapping with multilayer feedforward networks. Neural Netw., v.5, n.1, p.129-138. Citado na página 44.

Garey, M. R.; Johnson, D. S. (1990). Computers and Intractability : A Guide to the Theory of NP-Completeness. W. H. Freeman \& Co., New York, NY, USA. Citado nas páginas 70 e 72 . 
Goldberg, D. E. (1989). Genetic Algorithms in Search, Optimization and Machine Learning. Addison-Wesley Longman Publishing Co., Inc., Boston, MA, USA. Citado nas páginas 5, 73, e 119.

Golestani, A.; Motlagh, M. R. J.; Ahmadian, K.; Omidvarnia, A. H.; Mozayani, N. (2009). A new criterion to distinguish stochastic and deterministic time series with the Poincaré section and fractal dimension. Chaos, v.19, n.013137, p.1-13. Citado na página 18.

Gray, J. (2009). eScience: A Transformed Scientific Method. Hey, T.; Tansley, S.; Tolle, K., editores, The Fourth Paradigm: Data-Intensive Scientific Discovery. Microsoft Research. Citado nas páginas 1, 2, 3, e 65.

Hamilton, J. D. (1994). Time Series Analysis. Princeton University Press. Citado na página 26.

Hardy, G. H.; Wright, E. M. (1980). An Introduction to the Theory of Numbers. Oxford University Press, 5 edição. Citado na página 31.

Haykin, S. (2009). Neural networks and learning machines. Pearson Education Inc., 2nd edição. Citado na página 23.

Hegger, R.; Kantz, H.; Schreiber, T. (1998). Practical implementation of nonlinear time series methods: The TISEAN package. Chaos, v.9, p.413. Citado nas páginas 19, 23, 44, e 94 .

Hey, T.; Trefethen, A. E. (2005). Cyberinfrastructure for e-Science. Science, v.308, n.5723, p.817-821. Citado na página 2.

Hinterding, R. (2000). Representation, mutation and crossover issues in evolutionary computation. Proc. of the 2000 Congress on Evolutionary Computation, p. 916-923, Piscataway, NJ. IEEE Service Center. Citado na página 120.

Hopfield, J. J. (1982). Neural networks and physical systems with emergent collective computational abilities. Biophysics, v.79, p.2554-2558. Citado na página 5.

Huaiming, S.; Yang, W.; Mingyuan, A.; Weiping, W.; Ninghui, S. (2008). Query prediction in large scale data intensive event stream analysis systems. GCC '08: Proceedings of the 2008 Seventh International Conference on Grid and Cooperative Computing, p. 301-308, Washington, DC, USA. IEEE Computer Society. Citado na página 11.

Ishii, R. P.; de Mello, R. F. (2009). A history-based heuristic to optimize data access in distributed environments. 21st IASTED International Conference 
Parallell and Distributed Computing and Systems (PDCS2009), Cambridge, MA. Citado nas páginas 12, 74, 76, 79, e 90.

Ishii, R. P.; de Mello, R. F.; Yang, L. T. (2007). A Complex Network-Based Approach for Job Scheduling in Grid Environments. Perrott, R. H.; Chapman, B. M.; Subhlok, J.; de Mello, R. F.; Yang, L. T., editores, High Performance Computing and Communications, Third International Conference, HPC, v. 4782 de Lecture Notes in Computer Science. Springer, Houston, USA. Citado nas páginas 73 e 104.

Jacobson, V.; Leres, C.; McCanne, S. (2010). TCPDump Man Pages. Available at: Linux Systems, calling the man command for tcpdump. Citado na página 75 .

Jeffreys, H.; Jeffreys, B. S. (1988). Weierstrass's Theorem on Approximation by Polynomials and Extension of Weierstrass's Approximation Theory. Methods of Mathematical Physics. Cambridge University Press, England, 3rd edição. Citado na página 24.

Jung, C.; Woo, D.-K.; Kim, K.; Lim, S.-S. (2007). Performance characterization of prelinking and preloadingfor embedded systems. Proc. $7^{\text {th }}$ ACM \& IEEE Int. Conf. on Embedded Software, p. 213-220, New York, NY, USA. ACM. Citado nas páginas 75 e 88.

Katok, A.; Hasselblatt, B. (1995). Introduction to the Modern Theory of Dynamical Systems. Cambridge University Press, Cambridge. Citado na página 33.

Kennel, M. B.; Brown, R.; Abarbanel, H. D. I. (1992). Determining embedding dimension for phase-space reconstruction using a geometrical construction. Phys. Rev. A, v.45, n.6, p.3403-3411. Citado nas páginas 22, 23, e 27.

Kim, J.; Chandra, A.; Weissman, J. B. (2009). Using data accessibility for resource selection in large-scale distributed systems. IEEE Trans. Parallel Distrib. Syst., v.20, n.6, p.788-801. Citado nas páginas 4, 6, 9, 84, e 101.

Kirkpatrick, S.; Gelatt, C. D.; Vecchi, M. P. (1983). Optimization by simulated annealing. Science, v.220, 4598, n.4598, p.671-680. Citado nas páginas 5, 73 , e 122 .

Kraskov, A.; Stögbauer, H.; Andrzejak, R. G.; Grassberger, P. (2003). Hierarchical Clustering Based on Mutual Information. eprint arXiv:q-bio/0311039, v.1, p.1-11. Citado na página 23. 
Ladin, R.; Liskov, B.; Shrira, L.; Ghemawat, S. (1992). Providing high availability using lazy replication. ACM Trans. Comput. Syst., v.10, n.4, p.360-391. Citado na página 98.

Lee, T.-H.; White, H.; Granger, C. W. J. (1993). Testing for neglected nonlinearity in time series models: a comparison of neural network methods and alternative tests. Journal of Econometrics, v.56, p.269-290. Citado nas páginas 20 e 43.

Li, M.; Chen, W.-S.; ; Han, L. (2009). Correlation matching method for the weak stationarity test of 1rd traffic. Telecommunication Systems, v.43, n.3-4, p.181-195. Citado na página 21.

Liblit, B.; Naik, M.; Zheng, A. X.; Aiken, A.; Jordan, M. I. (2005). Scalable statistical bug isolation. PLDI '05: Proceedings of the 2005 ACM SIGPLAN conference on Programming language design and implementation, p. 15-26, New York, NY, USA. ACM. Citado na página 11.

Marwan, N. (2008). A historical review of recurrence plots. Eur. Phys. J. Special Topics, v.164, p.3-12. Citado nas páginas 18 e 30.

Marwan, N.; Carmen Romano, M.; Thiel, M.; Kurths, J. (2007). Recurrence plots for the analysis of complex systems. Physics Reports, v.438, n.5-6, p.237-329. Citado nas páginas xiii, 16, 18, 27, 29, 30, 31, 32, 33, e 35.

Mayer, U. F. (2010). Linux/unix nbench. http://www.tux.org/ mayer/linux/bmark.html. Citado na página 88.

Medina, A.; Lakhina, A.; Matta, I.; Byers, J. (2001). Brite: an approach to universal topology generation. Proc. $9^{\text {th }}$ Int. Symposium on Modeling, Analysis and Simulation of Computer and Telecommunication Systems, p. 346-353. Citado nas páginas 84,85 , e 87.

Mello, R. F.; Yang, L. T. (2009). Prediction of Dynamical, Non-Linear and Unstable Process Behavior. Journal of Supercomputing, v.49, p.22-41. Citado nas páginas 5 e 12 .

Morettin, P. A.; Toloi, C. M. C. (2006). Análise de Séries Temporais. Editora Edgard Blucher Ltda., São Paulo, 2 edição. Citado nas páginas xiii, 16, 17, $18,20,26,42$, e 43 .

Narayanan, D.; Donnelly, A.; Rowstron, A. (2008). Write off-loading: Practical power management for enterprise storage. Trans. Storage, v.4, n.3, p.1-23. Citado nas páginas 6, 50, 51, e 104. 
National Center, f. A. R. (2005). The Earth System Grid-ESG Project. www.earthsystemgrid.org. Citado na página 9.

Nielsen, M. (2009). A guide to the day of big data. Nature, v.462, p.722-723. Citado nas páginas 1 e 3.

Oldfield, R.; Kotz, D. (2006). Improving data access for computational grid applications. Cluster Computing, v.9, n.1, p.79-99. Citado nas páginas 4, 8 , e 101.

Oliker, L.; Biswas, R.; Shan, H.; Smith, W. (2004). Scheduling in heterogeneous grid environments: The effects of data migration. Proc. $12^{\text {th }}$ Int. Conf. on Advances in Computing \& Communications, Ahmedabad, INDIA. Citado nas páginas 4, 7, e 101.

Packard, N. H.; Crutchfield, J. P.; Farmer, J. D.; Shaw, R. S. (1980). Geometry from a time series. Phys. Rev. Lett., v.45, n.9, p.712-716. Citado na página 30.

Parashar, M.; Hariri, S. (2007). Autonomic Computing: Concepts, Infrastructure, and Applications / Editor(s): Manish Parashar and Salim Hariri. Taylor \& Francis, Inc., Bristol, PA, USA. Citado na página 2.

Provanzale, A.; Smith, L. A.; Vio, R.; Murante, G. (1992). Distinguishing between low-dimensional dynamics and randomness in measured time series. Phys. D, v.58, n.1-4, p.31-49. Citado na página 21.

Rahman, R. M.; Barker, K.; Alhajj, R. (2005). Replica selection in grid environment: a data-mining approach. Proc. Symposium on Applied Computing, p. 695-700, New York, NY, USA. ACM. Citado nas páginas $3,4,8$, e 101 .

Rahman, R. M.; Barker, K.; Alhajj, R. (2007). A predictive technique for replica selection in grid environment. Proc. $7^{\text {th }}$ IEEE Int. Symposium on Cluster Computing and Grid, p. 163-170. Citado na página 3.

Ranjan, R.; Harwood, A.; Buyya, R. (2006). A study on peer-to-peer based discovery of grid resource information. Relatório técnico, Department of Computer Science and Software Engineering University of Melbourne, Victoria, Australia. Citado nas páginas 2 e 3.

Rao, B. B. (2007). Deterministic and stochastic trends in the time series models: A guide for the applied economist. MPRA Paper 3580, University Library of Munich, Germany, Munich, Germany. Citado na página 18. 
Sato, H.; Matsuoka, S.; Endo, T. (2009). File clustering based replication algorithm in a grid environment. CCGRID '09: Proceedings of the 2009 9th IEEE/ACM International Symposium on Cluster Computing and the Grid, p. 204-211, Washington, DC, USA. IEEE Computer Society. Citado nas páginas 4, 9, e 101 .

Sato, H.; Matsuoka, S.; Endo, T.; Maruyama, N. (2008). Access-pattern and bandwidth aware file replication algorithm in a grid environment. GRID 'O8: Proceedings of the 2008 9th IEEE/ACM International Conference on Grid Computing, p. 250-257, Washington, DC, USA. IEEE Computer Society. Citado na página 9.

Schefler, W. C. (1988). Statistics: Concepts and Applications. Benjamin-Cummings Publishing Co., Inc., Redwood City, CA, USA. Citado nas páginas 57 e 89.

Schreiber, T.; Schmitz, A. (1997). Discrimination power of measures for nonlinearity in a time series. Phys. Rev. E., v.55, n.5443, p.5443-5447. Citado na página 19.

Semenov, M. A.; Terkel, D. A. (2003). Analysis of convergence of an evolutionary algorithm with self-adaptation using a stochastic lyapunov function. Evol. Comput., v.11, n.4, p.363-379. Citado na página 119.

Shumway, R. H.; Stoffer, D. S. (2006). Time Series Analysis and Its Applications: With $R$ Examples. Springer, 2nd edição. Citado nas páginas 16 e 26.

SMART (2010). Self-Monitoring, Analysis and Reporting Technology. Available at: http://en.wikipedia.org.wiki/s.m.a.r.t. Citado na página 75.

Souza, B. F. d.; de Carvalho, A. C. P. L. F.; Calvo, R.; Ishii, R. P. (2006). Multiclass svm model selection using particle swarm optimization. HIS '06: Proceedings of the Sixth International Conference on Hybrid Intelligent Systems, p. 31, Washington, DC, USA. IEEE Computer Society. Citado nas páginas 73 e 104.

Spillane, R. P.; Wright, C. P.; Sivathanu, G.; Zadok, E. (2007). Rapid file system development using ptrace. Proc. Workshop on Experimental Computer Science, p. 22, New York, NY, USA. ACM. Citado nas páginas 75, 76, e 88.

Stinchcombe, M.; White, H. (1989). Universal approximation using feedforward networks with non-sigmoid hidden layer activation functions. Proceedings of the International Joint Conference on Neural Networks, p. 612-617. Citado na página 44. 
Stockinger, H. (2007). Defining the Grid: a snapshot on the current view. The Journal of Supercomputing, v.42, p.3-17. Citado na página 2.

Sun, Y.; Xu, Z. (2004). Grid replication coherence protocol. Proc. $18^{\text {th }}$ Int. Symposium on Parallel and Distributed Processing, p. 232-239. Citado nas páginas $3,4,7,98$, e 101 .

Takens, F. (1981). Detecting strange attractors in turbulence. Dynamical Systems and Turbulence, v.1, p.366-381. Citado nas páginas 22 e 31.

Voß, S. (2001). Meta-heuristics: The state of the art. ECAI 'OO: Proceedings of the Workshop on Local Search for Planning and Scheduling-Revised Papers, p. 1-23, London, UK. Springer-Verlag. Citado na página 73.

Wang, C.-M.; Hsu, C.-C.; Chen, H.-M.; Wu, J.-J. (2006). Efficient multi-source data transfer in data grids. Proc. $6^{\text {th }}$ IEEE Int. Symposium on Cluster Computing and the Grid, p. 421-424, Washington, DC, USA. IEEE Computer Society. Citado nas páginas 8 e 101.

Wang, M.; Au, K.; Ailamaki, A.; Brockwell, A.; Faloutsos, C.; Ganger, G. R. (2004). Storage device performance prediction with cart models. MASCOTS '04: Proceedings of the The IEEE Computer Society's 12th Annual International Symposium on Modeling, Analysis, and Simulation of Computer and Telecommunications Systems, p. 588-595, Washington, DC, USA. IEEE Computer Society. Citado na página 10.

Wang, T.-L.; Gragg, W. B. (2003). Convergence of the unitary qr algorithm with a unimodular wilkinson shift. Math. Comput., v.72, n.241, p.375-385. Citado na página 24.

Ware, H.; Frederick, F. (2010). VMstat Man Pages. Available at: Linux Systems, calling the man command for vmstat. Citado na página 75.

White, H. (1989). An additional hidden unit test for neglected nonlinearity in multilayer feedforward networks. International Joint Conference on Neural Networks-IJCNN, v. 2, p. 451-455. Citado nas páginas 19, 20, 43, e 44.

White, H. (1990). Connectionist nonparametric regression: multilayer feedforward networks can learn arbitrary mappings. Neural Netw., v.3, n.5, p.535-549. Citado nas páginas 43 e 44.

Xu, W.; Huang, L.; Fox, A.; Patterson, D.; Jordan, M. I. (2009). Detecting large-scale system problems by mining console logs. SOSP '09: Proceedings of the ACM SIGOPS 22nd symposium on Operating systems principles, p. 117-132, New York, NY, USA. ACM. Citado na página 11. 
Yu, D.; Lu, W.; Harrison, R. G. (1998). Space time-index plots for probing dynamical nonstationarity. Physics Letters A, v.250, n.4-6, p.323- 327. Citado na página 21.

Zbilut, J. P.; Webber Jr., C. L. (1992). Embeddings and delays as derived from quantification of recurrence plots. Physics Letters A, v.171, n.3-4, p.199-203. Citado nas páginas 32 e 35. 


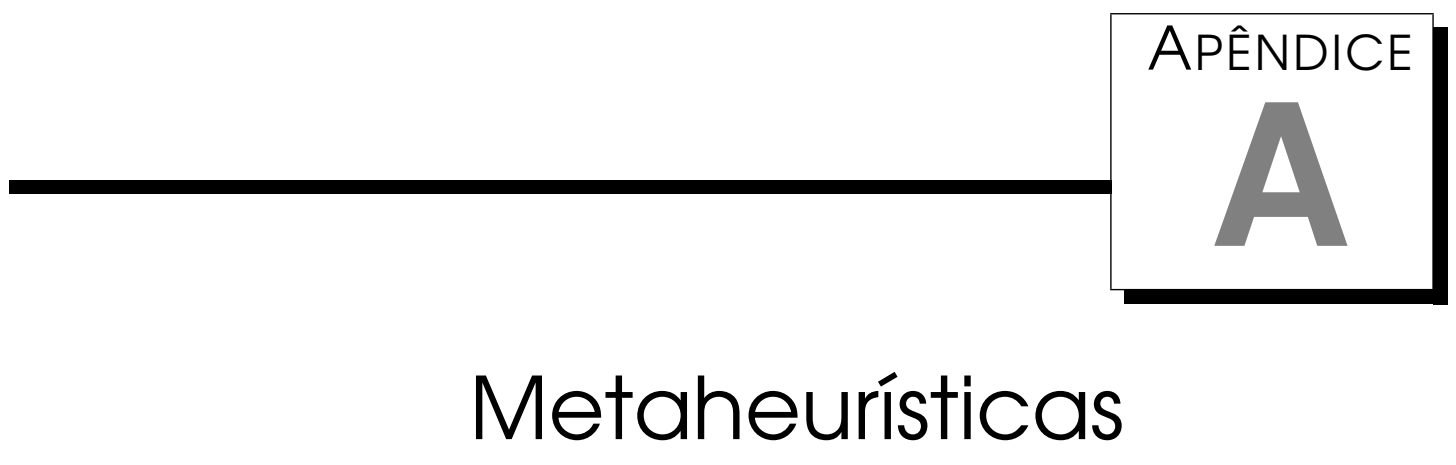

\section{A. 1 Algoritmos genéticos}

Algoritmos genéticos (AG) têm sido aplicados como métodos de busca e otimização em vários domínios (Goldberg, 1989; Semenov e Terkel, 2003). Esses algoritmos são baseados em mecanismos de seleção natural, visando a sobrevivência de indivíduos mais aptos. Essa abordagem não garante soluções ótimas para todas as instâncias do problema, mas fornece soluções apropriadas para uma quantidade razoável de problemas NP-Completos (Semenov e Terkel, 2003).

A resolução de problemas por meio de AG envolve dois aspectos: a codificação da solução em cromossomos e uma função de aptidão (fitness). Cada cromossomo representa uma tentativa de solução, no espaço de soluções possíveis.

Nessa tentativa de solucionar problemas, técnicas de codificação podem usar cadeias de bits, base binária, números reais etc. A função de aptidão é responsável por avaliar as possíveis soluções para o problema. Essa função recebe como entrada um cromossomo e devolve um número real, o qual representa seu grau de adaptabilidade, informando quão distante esse cromossomo está da solução ótima.

Os cromossomos (indivíduos) mais aptos são identificados e armazenados durante o processo de evolução. Os mais fracos, por outro lado, são excluídos. Vários métodos podem ser aplicados nessa escolha, dentre eles, as seleções proporcional, por ranking e por torneio (Back et al., 1999b,a).

Uma vez selecionados os indivíduos para reprodução, necessita-se 
de técnicas para modificação das características genéticas dos seus descendentes. Tais técnicas são conhecidas como operadores genéticos e os dois mais utilizados são o crossover e a mutação.

O operador crossover permite a troca de material genético entre dois indivíduos denominados pais, combinando informações de maneira que exista uma probabilidade razoável dos novos indivíduos serem melhores (Hinterding, 2000). O operador de um ponto é o mais utilizado. Para aplicá-lo, selecionam-se dois indivíduos (pais) e, a partir de seus cromossomos, são gerados dois novos indivíduos (filhos). Para gerar os filhos, seleciona-se um mesmo ponto de corte aleatoriamente nos cromossomos pais, e os segmentos criados são combinados.

Um exemplo de crossover de um ponto é apresentado na Figura A.1. A Figura A.1 (a) mostra os dois indivíduos selecionados, e o ponto escolhido é representado pelo símbolo “|”. A Figura A.1 (b) representa os novos indivíduos gerados após o cruzamento.

$$
\begin{array}{cc}
X_{1} X_{2} \mid X_{3} X_{4} X_{5} X_{6} & X_{1} X_{2} \mid Y_{3} Y_{4} Y_{5} Y_{6} \\
Y_{1} Y_{2} \mid Y_{3} Y_{4} Y_{5} Y_{6} & Y_{1} Y_{2} \mid X_{3} X_{4} X_{5} X_{6}
\end{array}
$$

\section{(a) Antes do cruzamento (b) Depois do cruzamento}

Figura A.1: Operador crossover

A mutação tem como objetivo substituir o valor de um gene por outro valor aleatório. No caso do indivíduo ser representado por uma cadeia de bits, ela consiste em escolher, aleatoriamente, um gene do cromossomo e inverter seu valor de 1 para 0 ou vice-versa. O propósito da mutação é manter a diversidade da população e assegurar que o cromossomo sempre cobrirá uma parte suficientemente grande do espaço de busca (Hinterding, 2000). Ela normalmente é aplicada em baixas frequências, pois em altas implicaria em busca aleatória.

\section{A.2 Colônia de formigas}

Biologicamente inspirada e baseada em população de insetos, a abordagem ACO permite a exploração de um amplo espaço de busca. Tarefas são distribuídas para formigas, que funcionam como agentes simplificados. Esses agentes podem se agrupar e constituir colônias, indicando soluções otimizadas para problemas complexos (Bonabeau et al., 2000; Dorigo e Di Caro, 1999).

Uma colônia de formigas é capaz de descobrir o menor caminho entre uma origem e um destino, compartilhando essa informação com as demais formigas (Dorigo e Di Caro, 1999). Stimergy é um mecanismo de comunicação 
utilizado pelas formigas para coordenar suas funções. Para tanto, as formigas depositam um rastro de uma substância química ao longo do caminho traçado durante seu movimento, denominada feromônio (Deneubourg et al., 1990). Essa substância é detectada e influencia as demais formigas. Uma formiga que se desloca de forma aleatória pode encontrar um rastro de feromônio deixado por outras. Dessa forma, esse rastro orienta uma formiga isolada, auxiliando-a na decisão de qual caminho seguir segundo sua probabilidade. Uma vez que a formiga escolhe o caminho com maior presença de feromônio, tal substância é reforçada aumentando a probabilidade de outras adotarem-no.

Considere quatro formigas $\left(F_{1}, F_{2}, F_{3}\right.$ e $\left.F_{4}\right)$ e dois caminhos que levam ao local de alimento $\left(C_{1}\right.$ e $\left.C_{2}\right)$ (Figura A.2). Suponha que o caminho $C_{1}$ seja maior que $C_{2}\left(C_{1}>C_{2}\right)$. Considere, ainda, que entre o local de origem e o local do alimento existam os nós: $N_{0}$ (nó origem) e $N_{D}$ (nó destino). Inicialmente todas as formigas estão no mesmo local de origem $\left(N_{O}\right)$ e devem escolher entre $C_{1}$ e $C_{2}$ para chegar até o alimento em $N_{D}$.

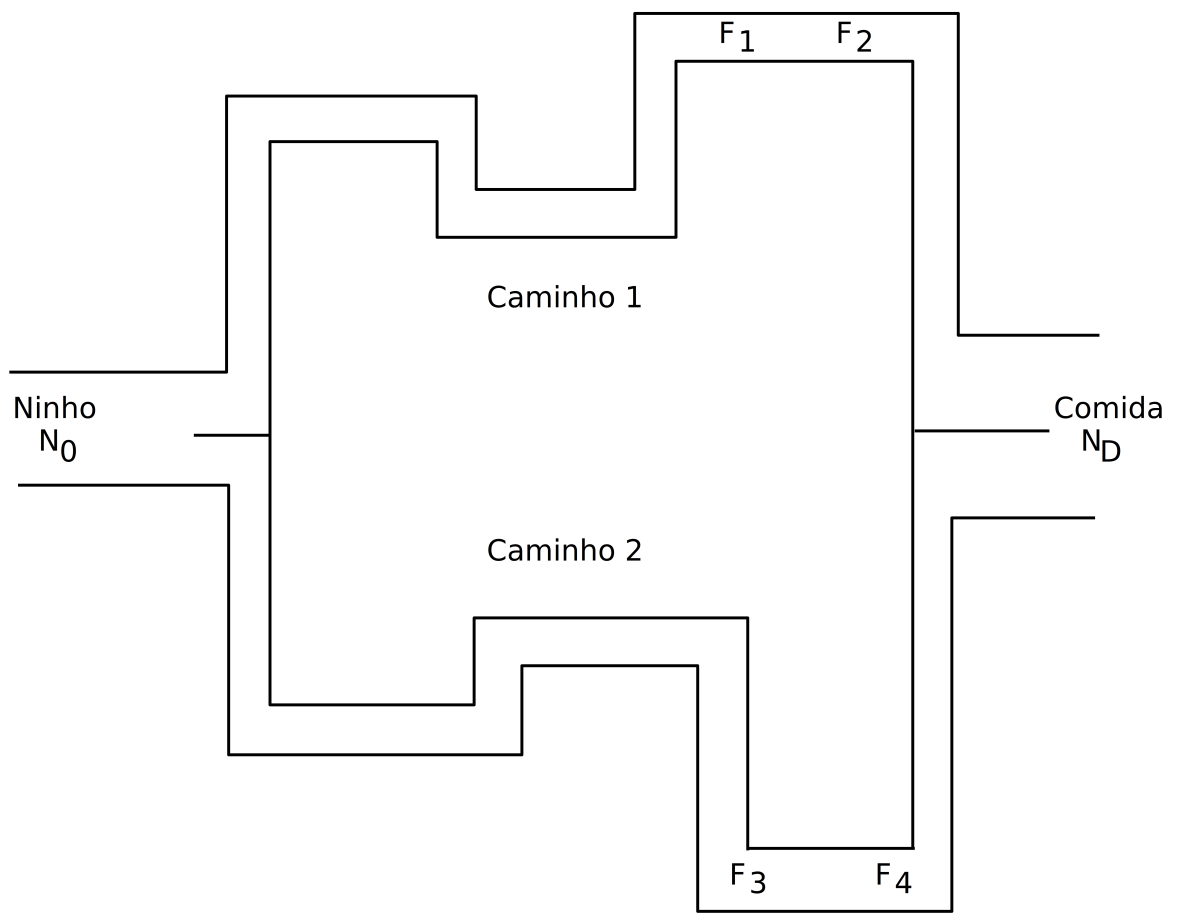

Figura A.2: Problema de busca ao alimento.

- $N_{O}$, nenhuma formiga tem conhecimento sobre o local $N_{D}$. Sendo assim, as formigas escolhem aleatoriamente um caminho, entre $C_{1}$ e $C_{2}$, para chegar a $N_{D}$. Suponha que $F_{1}$ e $F_{2}$ escolhem $C_{1}$, e $F_{3}$ e $F_{4}$ escolhem $C_{2}$;

- Pelo fato de $F_{1}$ e $F_{2}$ percorrerem o caminho $C_{1}$, e $F_{3}$ e $F_{4}$ percorrerem o caminho $C_{2}$, as formigas depositam uma quantidade de feromônio nos caminhos $\tau_{C_{1}}$ e $\tau_{C_{2}}$, respectivamente; 
- Sendo $C_{2}<C_{1}$, então $F_{3}$ e $F_{4}$ chegam ao local $N_{D}$ antes que $F_{1}$ e $F_{2}$. No instante que $F_{3}$ e $F_{4}$ chegam em $N_{D}, \tau_{C_{2}}=2$, mas $F_{1}$ e $F_{2}$ ainda permanecem no percurso $C_{1}$, portanto $\tau_{C_{1}}=0$. As formigas $F_{3}$ e $F_{4}$, estando em $N_{D}$, devem retornar a $N_{O}$. Em $N_{D}, F_{3}$ e $F_{4}$ detectam que $\tau_{C_{2}}>\tau_{C_{1}}$, portanto a probabilidade das formigas escolherem $C_{2}$ é maior. Suponha, então, que $C_{2}$ foi escolhido;

- Como $F_{3}$ e $F_{4}$ passam por $C_{2}$ pela segunda vez para alcançar $N_{D}, \tau_{C_{2}}$ é incrementado para 4. O aumento de feromônio em $\tau_{C_{2}}$ indica que $C_{2}$ é o menor caminho. Quando $F_{1}$ e $F_{2}$ chegarem em $N_{D}, \tau_{C_{2}}=4$ e $\tau_{C_{1}}=2$. Portanto, $F_{1}$ e $F_{2}$ são induzidos a escolherem o caminho $C_{2}$ para retornar a $N_{O}$.

Considerando o exemplo da Figura A.2, qualquer formiga é capaz de encontrar o caminho ótimo, uma vez que $F_{3}$ e $F_{4}$ alcançaram $N_{D}$, pois há maior quantidade de feromônio no caminho $C_{2}$. Uma formiga em $N_{O}$ seleciona aleatoriamente um caminho com probabilidades idênticas, dado que a quantidade de feromônio é nula.

\section{A.3 Simulated Annealing}

A técnica Simulated Annealing (SA) tem o objetivo de encontrar um mínimo global para uma função de energia que otimiza um dado problema (Kirkpatrick et al., 1983). O nome dessa técnica foi proposto como analogia à técnica de annealing em metalurgia, que consiste no aquecimento e resfriamento controlado de materiais com o objetivo de reduzir defeitos físicos.

Essa técnica introduz o conceito de temperatura do sistema, que possibilita encontrar mínimos globais. Para tanto, a cada iteração do algoritmo, o mecanismo considera um possível estado vizinho ao atual $S$, denominado $S^{\prime}$. Durante a avaliação, é determinada a probabilidade de transição do estado atual para um novo, sendo que o sistema tende a passar por estados que apresentem menor energia. A vizinhança é definida de maneira explícita, apresentando variações para cada problema específico.

A probabilidade de transição de estado é calculada por meio da função $P\left(E, E^{\prime}, T\right)$, onde $E$ e $E^{\prime}$ representam as energias $E(S)$ e $E\left(S^{\prime}\right)$ dos estados correspondentes a $S$ e $S^{\prime}$, e o parâmetro $T$ corresponde à temperatura do sistema. A propriedade essencial da função é a probabilidade de transição $P$ ser diferente de zero, $\operatorname{com} E_{0}>E$ indicando uma transição para um estado pior (i.e., que apresenta maior energia). Essa característica do sistema é utilizada como forma de prevenir o sistema de estagnar em um mínimo local.

Por outro lado, se a temperatura $T$ é próxima de zero, a função de probabilidade também aproxima-se de zero para $E_{0}>E$, ou a função devolve 
um valor positivo, para $E_{0}<E$. Isso garante que, para valores pequenos de $T$, o sistema apresenta tendência de encontrar soluções com menor energia. Dessa maneira, a técnica SA pode ser utilizada para determinar mudanças de comportamento, fornecendo uma solução global adequada. Para aplicar essa técnica, entretanto, deve-se conhecer, profundamente, o sistema a fim de se determinar a variação de temperatura em busca do mínimo global. 